\title{
Sinusoidal Parameter Estimation and Application to Eddy Current NDT Data Records
}

\author{
by \\ Hossein Chahrour, B.Eng, Carleton University
}

A thesis submitted to the

Faculty of Graduate Studies and Postdoctoral Affairs in partial fulfillment of the requirements for the degree of

Master of Applied Science in Electrical and Computer Engineering

Ottawa-Carleton Institute for Electrical and Computer Engineering

Department of Systems and Computer Engineering

Carleton University

Ottawa, Ontario, Canada

(C)2014 Hossein Chahrour 


\section{Abstract}

The general problem of assessing eddy current data from steam generators is complicated due to the presence of random noise and low frequency sinusoidal interference signals. The low frequency sinusoidal interference originates from periodic variations in the material properties or dimensions caused by manufacturing processes, behaviour of the component of the inspection system such as probe wobbling. The estimation of the sinusoidal parameters is obtained by using the cyclic-MUSIC algorithm which is a combination of two well known algorithms, Relaxed and MUSIC algorithms. The removal process generates waveforms from the estimated parameters and subtracts them from the original data. Application to several data records showed that using all the estimated parameters with two sinusoidal waveforms is the best fit due to the nature of the data. Moreover, the performance assessment was based on power measurements and correlation functions. 


\section{Acknowledgements}

Many people over the years have helped me get here, so there are many people I need to thank. Firstly, I would like to thank my supervisor Professor Mohamed El-Tanany from Carleton University, for his support, encouragement, patience and advice throughout this research.

I would like to extend my gratitude to AECL for funding this research, and for providing me with the necessary data to achieve the research results.

Special thanks to Mr. Richard Lakhan for his help, patience, valuable time, and advice throughout the research, and for letting me ask many, many questions about the data and its setup.

Also, I would like to thank the AECL Team for providing me with the necessary documents to gain in-depth knowledge about Steam Generators and collection of Eddy Current data. 


\section{Table of Contents}

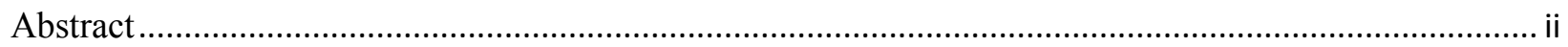

Acknowledgements ............................................................................................................................ ii

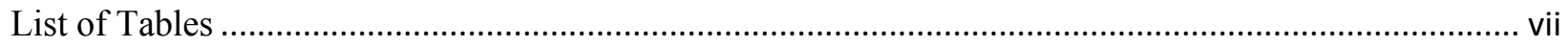

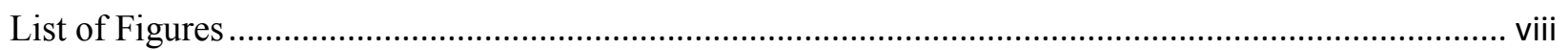

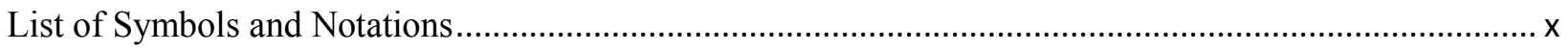

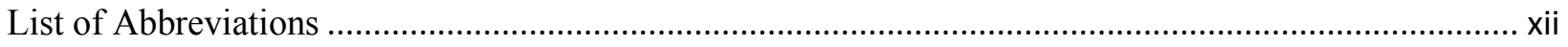

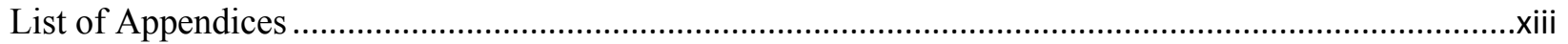

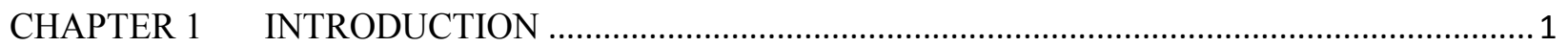

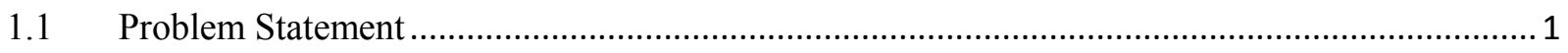

1.2 Steam Generator in Nuclear Power Plant.................................................................................. 2

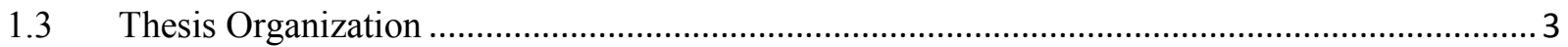

CHAPTER 2 NDT TECHNIQUES AND SG TUBE INSPECTION ................................................ 6

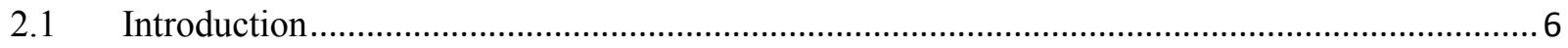

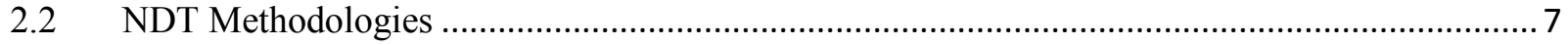

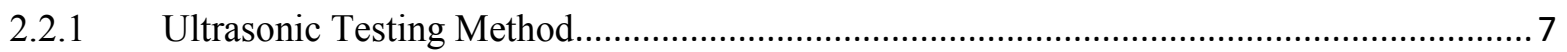

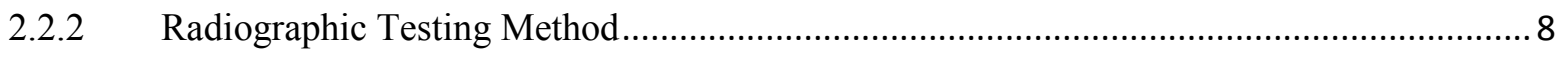

2.2.3 Electromagnetic Testing Method .................................................................................. 9

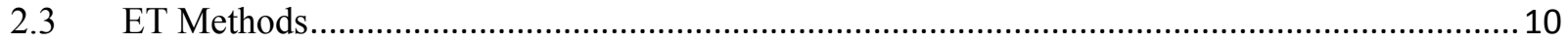

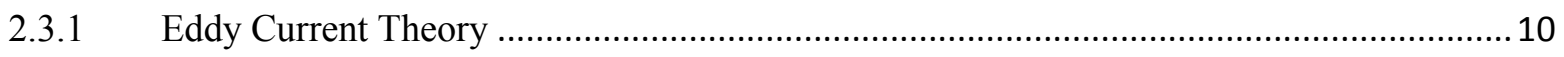

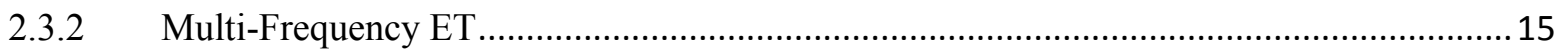

2.3.3 Remote Field Eddy Current Method .......................................................................... 16

2.3.4 Swept Frequency Eddy Current Method......................................................................... 17

2.3.5 Pulsed Eddy Current Method .................................................................................... 17

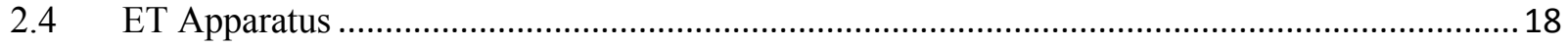

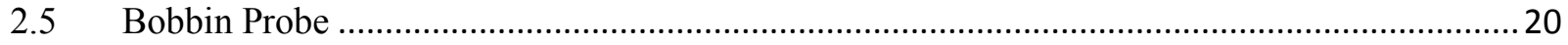

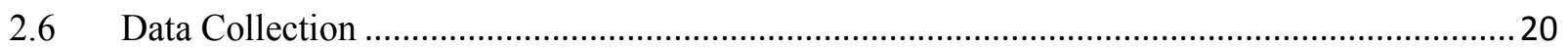

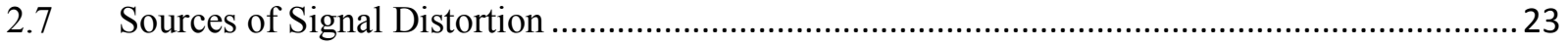

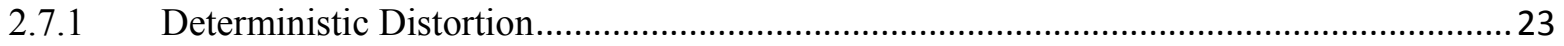

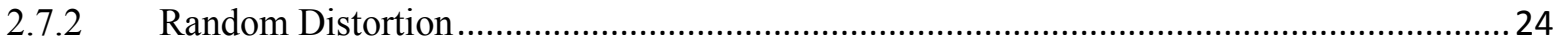




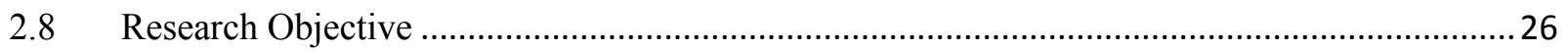

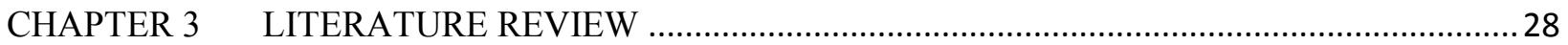

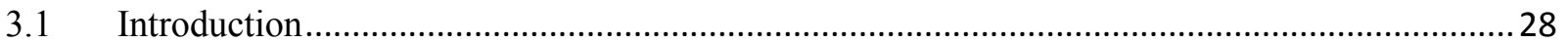

3.2 Dynamic Thresholding for Automated Analysis of Bobbin Probe Eddy Current Data ..............28

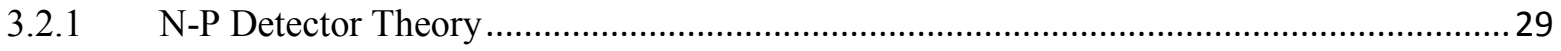

3.3 An Adaptive Wiener Filtering Based Technique for an Automated Detection System..............30

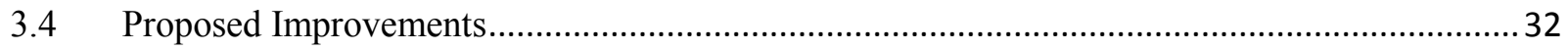

\section{CHAPTER 4 SIGNAL PREPROCESSING AND SINUSOIDAL PARAMETERS ESTIMATION} ALGORITHMS 33

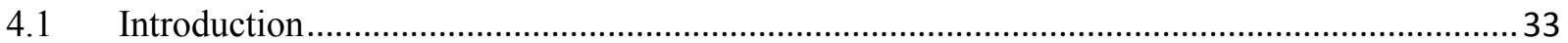

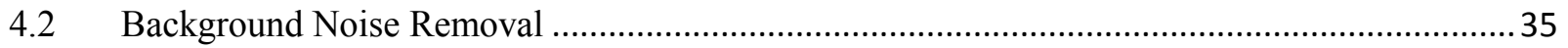

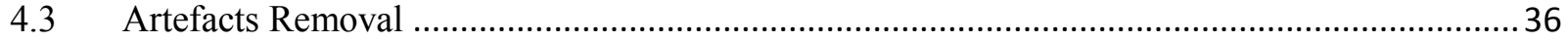

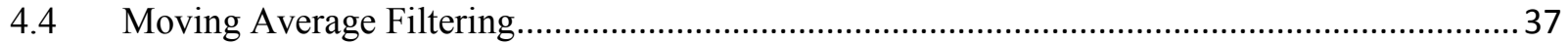

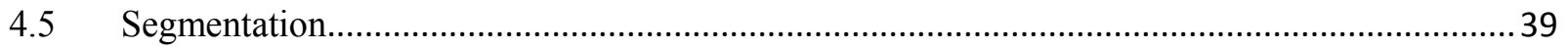

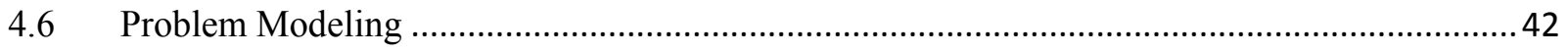

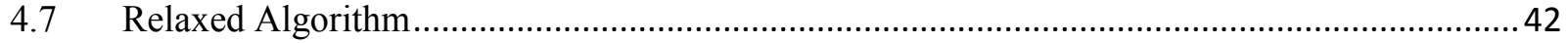

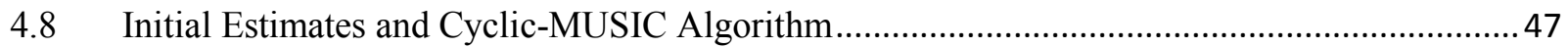

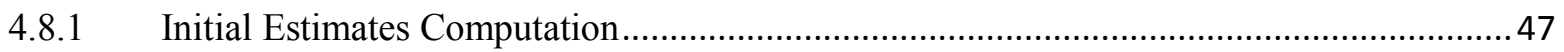

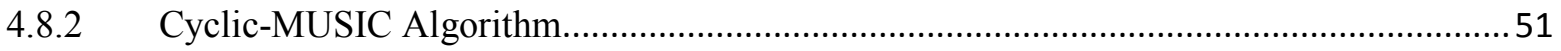

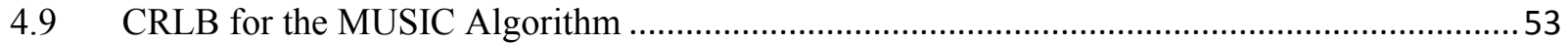

4.9.1 Asymptotic Covariance Matrix for Root-MUSIC .........................................................5

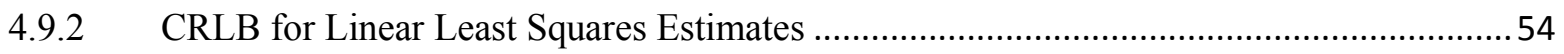

CHAPTER 5 IMPLEMENTATION AND RESULTS …............................................................... 56

$5.1 \quad$ Cyclic-MUSIC Algorithm Implementation …..................................................................... 56

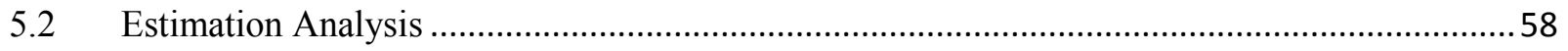

5.2.1 Improved Estimation using Rectangular Windowing ........................................................62

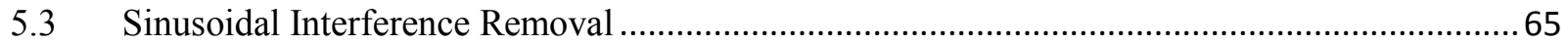

5.3.1 Using all Estimated Sinusoidal Parameters.................................................................... 65

5.3.2 Using the Tube Average Frequency for Each Segment ...............................................69

5.3.3 Using Tube Average Frequency and Magnitude for the Whole Data Record ................... 70

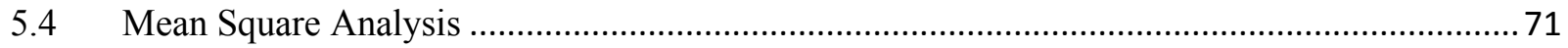

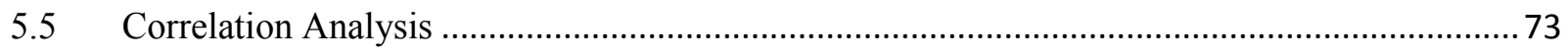




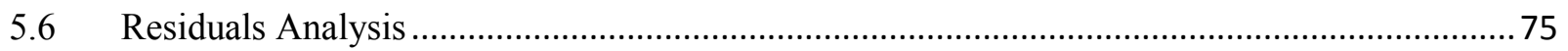

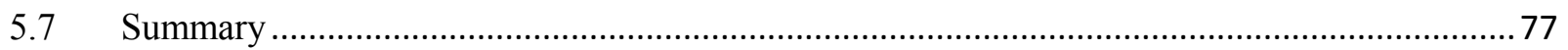

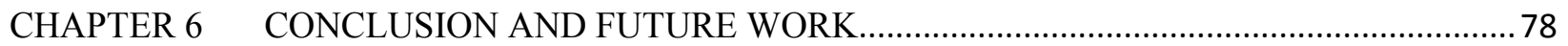

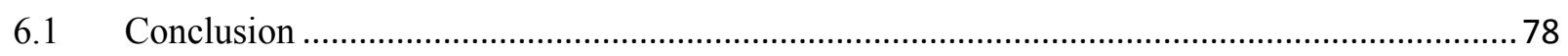

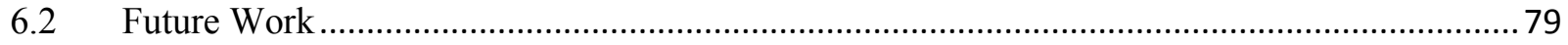

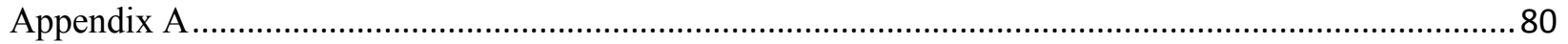

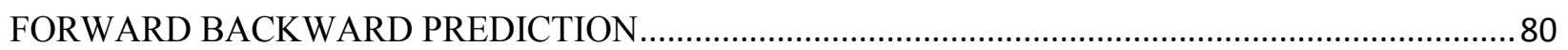

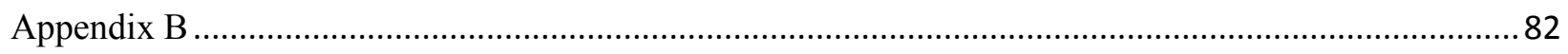

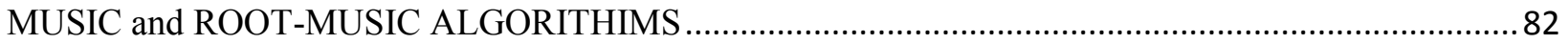

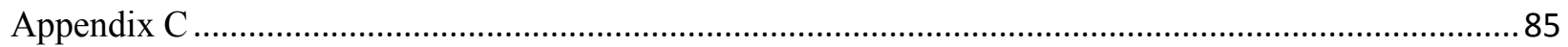

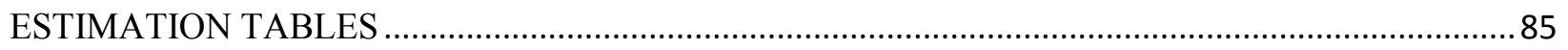

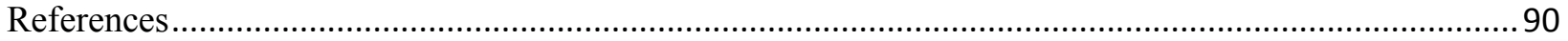




\section{List of Tables}

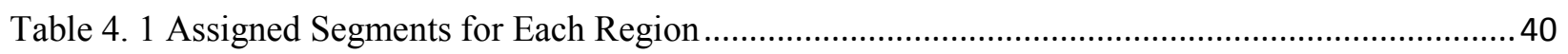

Table 5. 1 Frequencies, Magnitudes and Phases for One Sinusoid Estimation (TUBE 1) .......................59

Table 5. 2 Frequencies, Magnitudes and Phases for Two Sinusoids Estimation (TUBE 1) ......................60

Table 5. 3 Frequencies, Magnitudes and Phases for Four Sinusoids Estimation (TUBE 1)....................60

Table 5. 4 Frequencies, Magnitudes and Phases for One Sinusoid Estimation (TUBE 2) .......................61

Table 5. 5 Frequencies, Magnitudes and Phases for Two Sinusoids Estimation (TUBE 2) ....................61

Table 5. 6 Frequencies, Magnitudes and Phases for Four Sinusoids Estimation (TUBE 2).....................6 62

Table 5. 7 Improved Frequency Estimation for Two Sinusoids ...........................................................64

Table 5. 8 Mean Square Using All Estimated Parameters for One, Two and Four Sinusoids (TUBE 1).. 72

Table 5. 9 Mean Square Using Tube Average Frequency for Two Sinusoids (TUBE 1)....................... 72

Table 5. 10 Improved Power for the Truncated Peak Method .............................................................. 73

Table 5. 11 Correlation Coefficients Before and After the Removal of Low Frequency Sinusoidal Noise

Using all Estimated Parameters and Tube Average Frequency Techniques............................................. 74 


\section{List of Figures}

Figure 1. 1 CANDU Nuclear Reactor ........................................................................................... 4

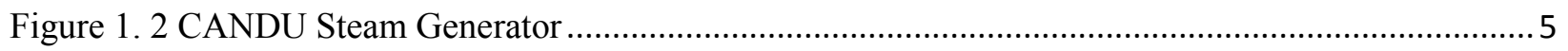

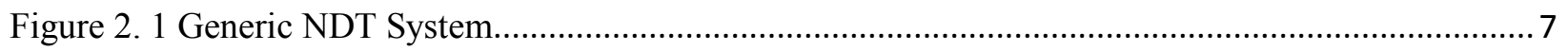

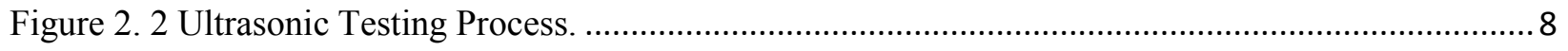

Figure 2. 3 Radiographic Testing Process. ................................................................................... 9

Figure 2. 4 Eddy Current Magnetic Fields Interactions...................................................................... 11

Figure 2. 5 Lift Off Curves a) Nonferromagnetic conductive material b) Ferromagnetic conductive

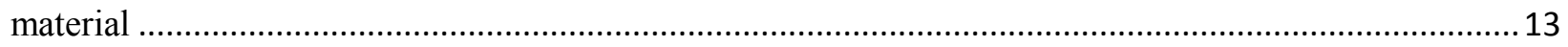

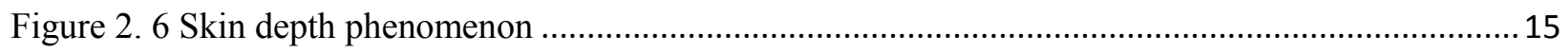

Figure 2. 7 Eddy Current Basic Testing Apparatus ........................................................................... 19

Figure 2. 8 Differential Bobbin Probe Scanning a Heat Exchanger Tube ...............................................2 21

Figure 2. 9 Typical Eddy Current Data Record for a) Vertical Channel and b) Horizontal Channel for 240

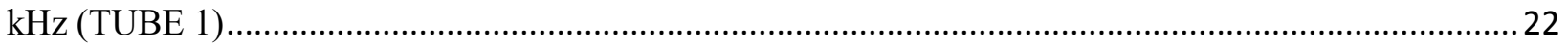

Figure 2. 10 Flaw Signals in Eddy Current Record at 480, 240 And 120 kHz (TUBE 1) .......................23

Figure 2. 11 Support Plate Responses at 480, 240 and $120 \mathrm{KHz}$ (TUBE 1)...........................................25

Figure 2. 12 Probe Wobble Response (TUBE 1) .............................................................................2

Figure 2. 13 Block Diagram of the Noise Removal Process..................................................................2

Figure 3. 1 (a): Original eddy current signal after high pass filtering (at the top), (b) Signal enhanced by LAWF(middle) (c) Signal enhanced by WAWF (end) [13] .............................................................. 31

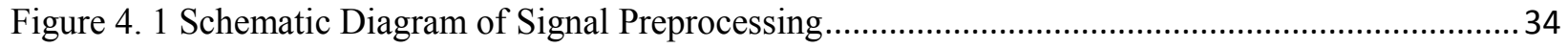

Figure 4. 2 Background Removal Block Diagram.............................................................................. 35

Figure 4. 3 Data Record Before and After Background Removal .......................................................... 36

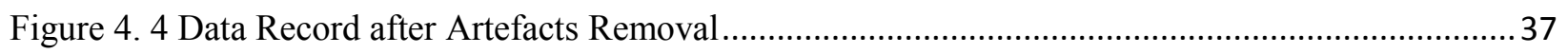

Figure 4. 5 Frequency Response of 3-Points Moving Average Filter...................................................... 38

Figure 4. 6 Data Record Before and After Moving Average Filtering .................................................. 38

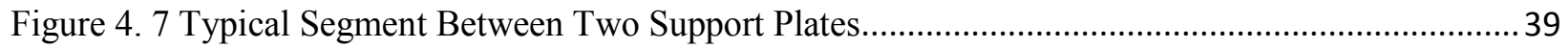

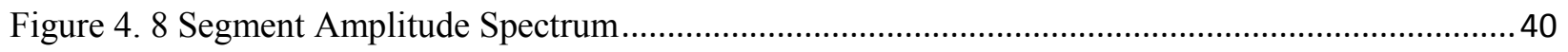

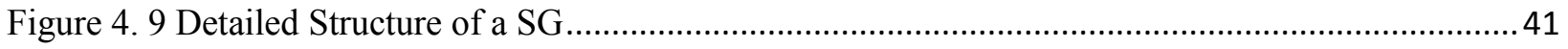

Figure 4. 10 Flowchart for Relaxed Algorithm........................................................................... 46

Figure 4. 11 Flowchart of the Initial Conditions Estimation Process ....................................................5 50

Figure 4. 12 Flowchart of Cyclic-MUSIC Algorithm......................................................................... 52

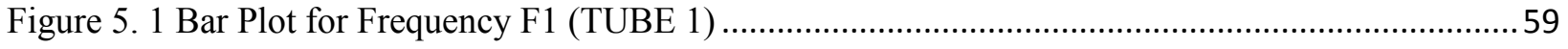

Figure 5. 2 Bar Plot for Magnitude M1 (TUBE1) ............................................................................5 59

Figure 5.3 a) Segment 6 b) Segment 17 c) Segment 18 (TUBE 1) .......................................................63

Figure 5. 4 a) Original Segment b) Excluding Peak Method c) Truncating Peak Method (TUBE 1) ........64

Figure 5. 5 Original Segment and Estimated Waveform for a) Original Estimation b) Excluding Peak

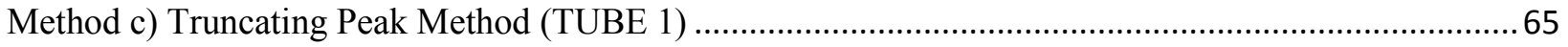


Figure 5. 6 Removal Process For One Sinusoid a) Original Segment b) Original Segment and Estimated

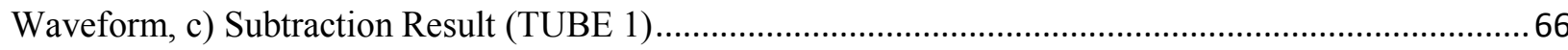
Figure 5. 7 Removal Process for Two Sinusoids a) Original Segment b) Original Segment and Estimated

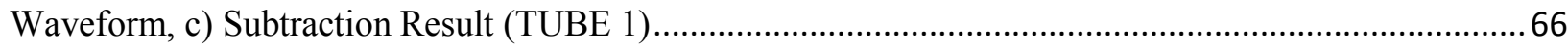
Figure 5. 8 Removal Process for Four Sinusoids a) Original Segment b) Original Segment and Estimated Waveform, c) Subtraction Result (TUBE 1).

Figure 5. 9 Removal Process for One Sinusoid a) Original Segment b) Original Segment and Estimated Waveform, c) Subtraction Result (TUBE 2) ............................................................................67

Figure 5. 10 Removal Process for Two Sinusoids a) Original Segment b) Original Segment and Estimated Waveform, c) Subtraction Result (TUBE 2) ..................................................................................68

Figure 5. 11 Removal Process for Four Sinusoids a) Original Segment b) Original Segment and Estimated

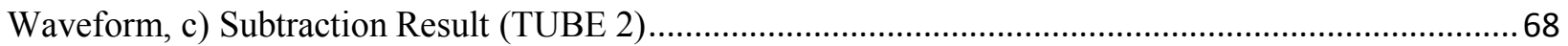

Figure 5. 12 Tube Average Frequency Technique a) Original Segment b) Original Segment and Estimated

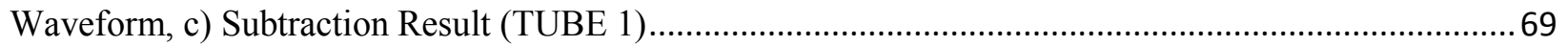

Figure 5. 13 Bar Plot for The Magnitude of First Frequency …............................................................ 70

Figure 5. 14 Bar Plot for The Magnitude of Second Frequency ............................................................ 70

Figure 5. 15 Tube Average Frequency and Magnitude a) Original Data Record and Estimated Waveform,

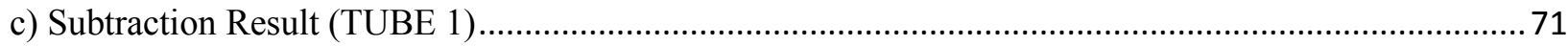

Figure 5. 16 Before and After the Removal Of Sinusoids Using All Estimated Parameters a) Flaw Signal b) One Sinusoid c) Two Sinusoids And d) Four Sinusoids (TUBE 1) ...................................................... 74

Figure 5. 17 Before and After the Removal Of Sinusoids Using Tube Average Frequency Technique a)

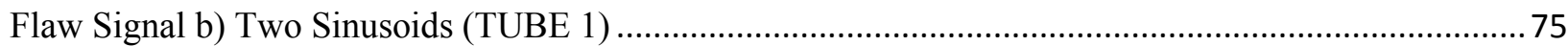

Figure 5.18 a) Histogram of All Segments and Residuals After Removing b) One Sinusoid c) Two

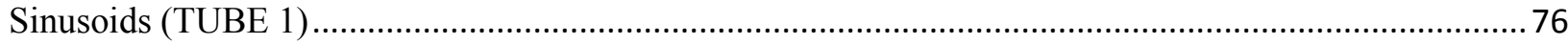
Figure 5. 19 a) QQ Plot of the Original Data b) QQ Plot After Removing One Sinusoid c) QQ Plot After

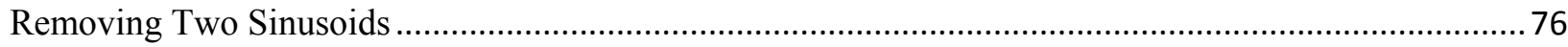




\section{List of Symbols and Notations}

\begin{tabular}{|c|c|}
\hline$\nabla$ & Curl operator \\
\hline$H$ & Magnetic field \\
\hline$J$ & Current density \\
\hline$\varepsilon$ & Electromotive force \\
\hline$\Phi$ & Magnetic flux \\
\hline$E$ & electrical field \\
\hline$B$ & Magnetic field \\
\hline$L$ & Inductance of a coil \\
\hline$I$ & Current source \\
\hline$\delta$ & Standard depth penetration \\
\hline$\mu$ & Magnetic permeability \\
\hline$f$ & Excitation frequency \\
\hline$\sigma$ & Electrical conductivity \\
\hline$\theta(n)$ & Measured signal \\
\hline$S(n)$ & Defect signal \\
\hline$W(n)$ & Additive white Gaussian noise \\
\hline$X$ & Data vector \\
\hline$W \sim \mathrm{N}\left(0, \sigma_{\omega}^{2}\right)$ & Normally distributed with 0 mean and $\sigma_{\omega}^{2}$ variance \\
\hline$S \sim \mathrm{N}\left(\mu_{\mathrm{s}}, \mathrm{C}_{\mathrm{s}}\right)$ & Multivariate normally distributed with $\mu_{\mathrm{s}}$ mean and $\mathrm{C}_{\mathrm{s}}$ covariance \\
\hline$T(X)$ & Test statistics \\
\hline$\lambda$ & Threshold value \\
\hline$C_{s}$ & Covariance matrix \\
\hline$\mu_{s}$ & Mean vector \\
\hline$\sigma_{\omega}^{2}$ & Additive white Gaussian noise variance \\
\hline$\sigma_{s}^{2}$ & Measured signal variance \\
\hline$y_{n}$ & Sampled data signal \\
\hline$e_{n}$ & Sample noise vector \\
\hline$\left|\alpha_{k}\right|$ & Real valued amplitude for $k^{\text {th }}$ sinusoid \\
\hline
\end{tabular}




\begin{tabular}{|c|c|}
\hline$\phi_{k}$ & Phase for $k^{\text {th }}$ sinusoid \\
\hline$\theta_{k}$ & Frequency for $k^{\text {th }}$ sinusoid \\
\hline$(.)^{T}$ & $T$ is the transpose \\
\hline$(.)^{H}$ & $H$ is the conjugate transpose \\
\hline$\|\cdot\|$ & Euclidean norm \\
\hline$\hat{\alpha}_{k}$ & Magnitude and phase estimates of the $k^{\text {th }}$ sinusoid \\
\hline$\hat{\theta}_{k}$ & Frequency estimate of the $k^{\text {th }}$ sinusoid \\
\hline$\hat{y}_{i}$ & Contains the $i^{t h}$ sinusoidal vector \\
\hline$X_{\text {mod }}$ & Correlation matrix \\
\hline$P_{X X}^{M U S I C}(f)$ & MUSIC spectrum estimate \\
\hline$s(f)$ & Complex sinusoidal vector \\
\hline$V$ & Noise subspace matrix \\
\hline$A$ & Linear regression matrix \\
\hline$\chi$ & Least squares estimates \\
\hline$\backslash$ & Left division matrix operation \\
\hline$V_{\text {mag }}$ & Estimated magnitudes vector \\
\hline$P_{\text {phase }}$ & Estimated phases vector \\
\hline$X_{p}$ & Magnitude of the peak \\
\hline$X_{i}$ & Element $i$ of the segment \\
\hline$m$ & Mean of the segment \\
\hline
\end{tabular}




\section{List of Abbreviations}

\begin{tabular}{ll}
\hline Abbreviation & Meaning \\
\hline AWF & Adaptive Wiener Filter \\
ERLB & Cramer-Rao Lower Bound \\
FFT & Eddy Current Testing \\
LAWF & Fast Fourier Transform \\
MUSIC & Lee Adaptive Wiener Filter \\
NDT & Multiple Signal Classification \\
PEC & Non-destructive Testing \\
PFA & Pulsed eddy current \\
N-P & Probability of false alarm \\
RFEC & Neyman-Pearson \\
SG & Remote field eddy current \\
WAWF & Steam Generator used in Nuclear Power Plant \\
\hline
\end{tabular}




\section{List of Appendices}

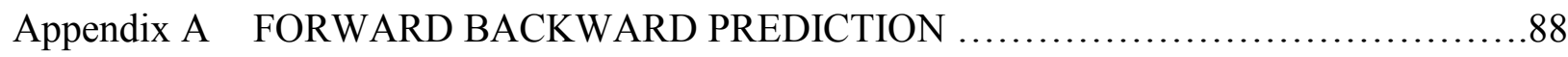

Appendix B MUSIC and ROOT-MUSIC ALGORITHIMS $\ldots \ldots \ldots \ldots \ldots \ldots \ldots \ldots \ldots \ldots \ldots \ldots \ldots$

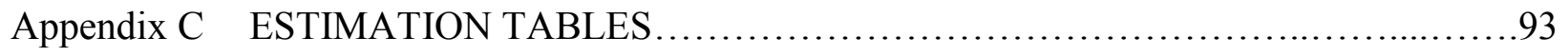




\section{CHAPTER 1 INTRODUCTION}

\subsection{Problem Statement}

Eddy current testing (ET) is one of the most extensively used non-destructive testing (NDT) techniques for inspecting electrically conductive materials at very high speeds. For example, ET is utilized in the NDT inspection for the tubing of stream generators (SGs) that are used in some nuclear power plants. The analysis of the resulting test data is becoming more important as nuclear power plants age, and their repairs are becoming more expensive.

Eddy current data analysis is complex due to presence of random noise and low frequency sinusoidal interference signals. In addition more difficulties arise from unwanted responses which are caused by support structures and geometry variations. Such responses may appear similar to defect signals, and as a result the task of detecting defect responses becomes more difficult.

This thesis proposes a solution for estimating and removing the low frequency sinusoidal interference using well know DSP algorithms. This solution consists of three stages preprocessing, interference estimation and interference cancellation. The expected outcome of this research is eddy current data records free of low-frequency sinusoidal noise while attempting to retain all the features of the data record and increase the probability of flaw detection. A more detailed description of the work addressed in this thesis is given in section 2.8 . 


\subsection{Steam Generator in Nuclear Power Plant}

Heat exchanger tubes are used in a wide range of industries, including power stations, petrochemical plants, oil refineries, air conditioning and refrigeration. Heat exchangers are called SGs, which are considered major components in pressurized nuclear power plant. SGs are one of the main barriers between the radioactive primary-side of the nuclear power plant and the non-radioactive secondary-side, and regular inspections of the SGs using NDT is required to prevent release of radiation to the plant and the surrounding environment. SGs generate steam from water using the heat generated from the reactor core in order to run the turbines to generate electricity $[1,2]$, as shown in Figure 1.1.

Figure 1.2 shows the internal structure and design of a stream generator. Typical SGs contain between 2600 to 4800 tubes each, and each reactor may have 4,8 , or 12 SGs. These tubes are typically made of Alloy 400, Alloy 600 or Alloy 800 which are all nickel based alloys containing varying levels of iron and chromium, and are resistant to corrosion at high temperatures. In addition, a typical tube is approximately $15 \mathrm{~m}$ long with an internal diameter of $15.5 \mathrm{~mm}$ and $1 \mathrm{~mm}$ wall thickness. The steam-generator tube-bundle, shown in Figure 1.2, has three main components which are the tube-sheet ${ }^{1}$, tubes, and support plates. The tubes are inserted into the tube sheet, and expanded to create a rolled joint and seal-welded [2].

As shown in figure 1.2, primary coolant ${ }^{2}$ enters the hot-leg tube end at $\mathrm{D}_{2} \mathrm{O}$ Inlet and flows up the hot-leg around the U-bend, down the cold-leg, and exits at the cold-tube end at $\mathrm{D}_{2} \mathrm{O}$ Outlet. The heat generated is transferred through the steam Outlet Nozzle, and generates steam which runs the turbine to drive the electrical generator as shown in Figure1.1.

\footnotetext{
${ }^{1}$ A tube sheet is a plate, which is perforated with a pattern of holes designed to accept pipes or tubes

${ }^{2}$ Primary coolant in a nuclear reactor used to remove heat from the nuclear reactor core and transfer it to electrical generator.
} 
The SG tubes are continuously exposed to harsh environmental conditions including high temperatures, pressures and material interactions. These conditions may result in various types of degradation mechanisms such as mechanical wear between tubes and tube supports, outer diameter stress corrosion ${ }^{3}$ cracking (ODSCC), pitting ${ }^{4}$, thinning, primary-water stress corrosion cracking 5 (PWSCC), and inter granular $\operatorname{attack}^{6}$ (IGA). Consequently, the SGs are inspected periodically for degradation to avoid tube leaks and to monitor the condition of the tubes [6].

SG tube inspection can be complex and challenging. There have been several cases of unscheduled plant shutdowns in the past which result in large economic losses for the plant. Visual examination and ultrasonic techniques have limited use as they are very slow and time consuming. Eddy current probing techniques offer a more flexible, faster and more economically viable alternative. However, as will be elaborated in next chapter, the ET records are corrupted by random noise and periodic interference which makes the data analysis less straight forward. Hence there is a strong financial incentive to develop dependable NDT techniques with digital signal processing to inspect SG tubes. [6].

\subsection{Thesis Organization}

This thesis investigates signal processing techniques that are suitable for the estimation of periodic interference that is commonly encountered in ET data records. The interference is attributed to a number of factors which include support plates, probe wobble, deposits and geometric variations. Removal of unwanted interference and noise require classification of noise and the use of appropriate DSP algorithms.

\footnotetext{
${ }^{3}$ Corrosion is the gradual destruction of materials, usually metals, by chemical reactions.

${ }^{4}$ Pitting is a form of extremely localized corrosion that leads to the creation of small holes in the metal.

${ }^{5}$ Stress corrosion cracking is the growth of crack formation in a corrosive environment.

${ }^{6}$ Inter granular attack is a form of corrosion where boundaries of crystallites of the material are more susceptible to corrosion than their insides.
} 


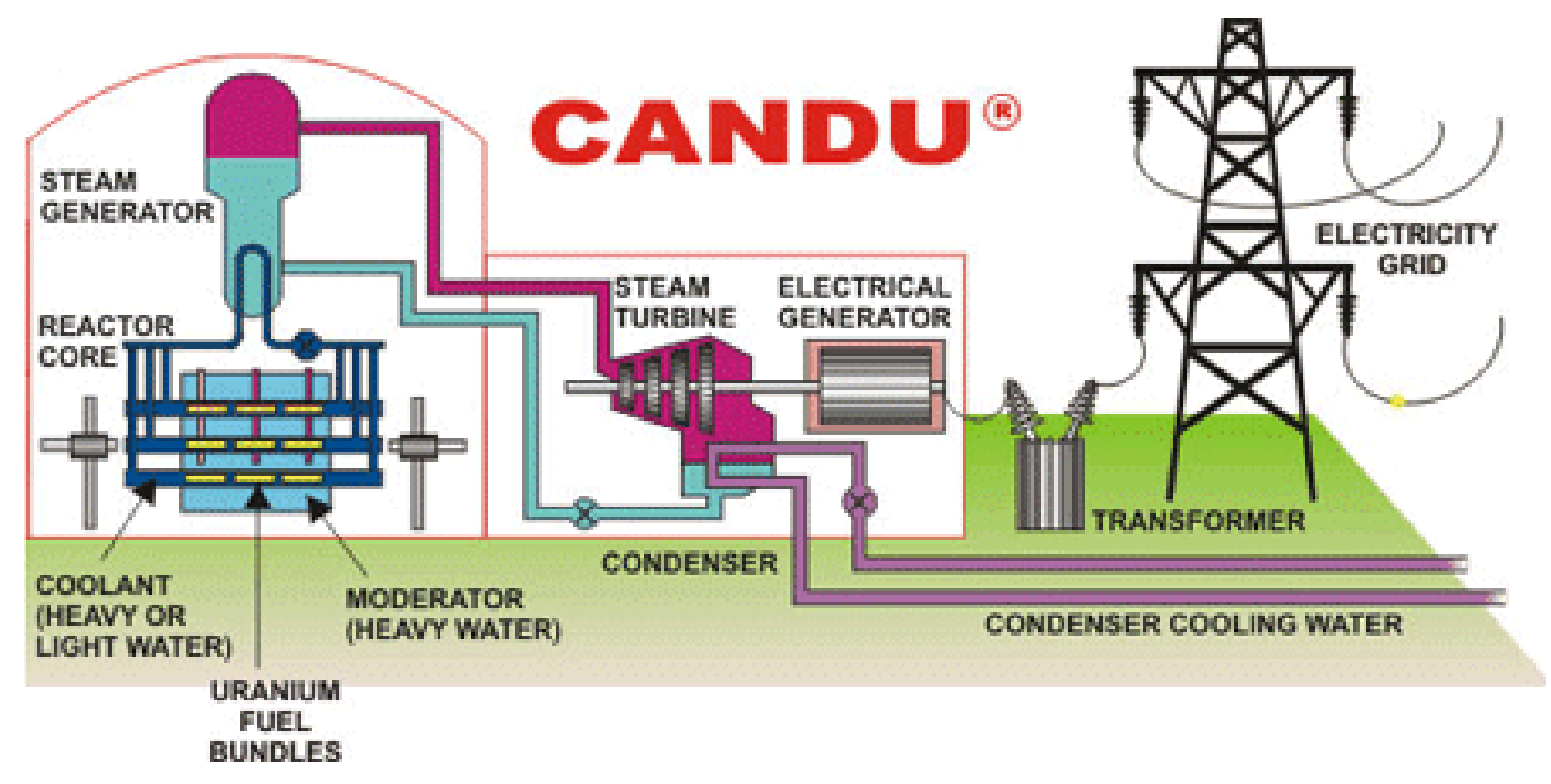

Figure 1. 1 CANDU Nuclear Reactor . Reproduced with permission Canadian Nuclear Association [3].

Specifically, the thesis investigates optimal and suboptimal algorithms to estimate the sinusoidal interference present in the test records, and uses the subtraction method to remove the sinusoidal interference.

The rest of the thesis is organized as follows.

Chapter 2 introduces the different types of NDT techniques currently in practice and then focuses on the eddy current NDT technique and describes the ET and its application to the inspection of SG tubes, focusing on the multi-frequency bobbin probe inspection methods. Chapter 3 summarises previous research in the eddy current data analysis field and how it is related to the current work. Signal preprocessing steps, which include background cleaning, artefacts removal, smoothing and segmenting processes, and sinusoidal parameters estimation using the Cyclic-MUSIC algorithm are discussed in Chapter 4. Cyclic-MUSIC algorithm 
implementation, results and summary of the research with future work are discussed in Chapters 5 and 6.

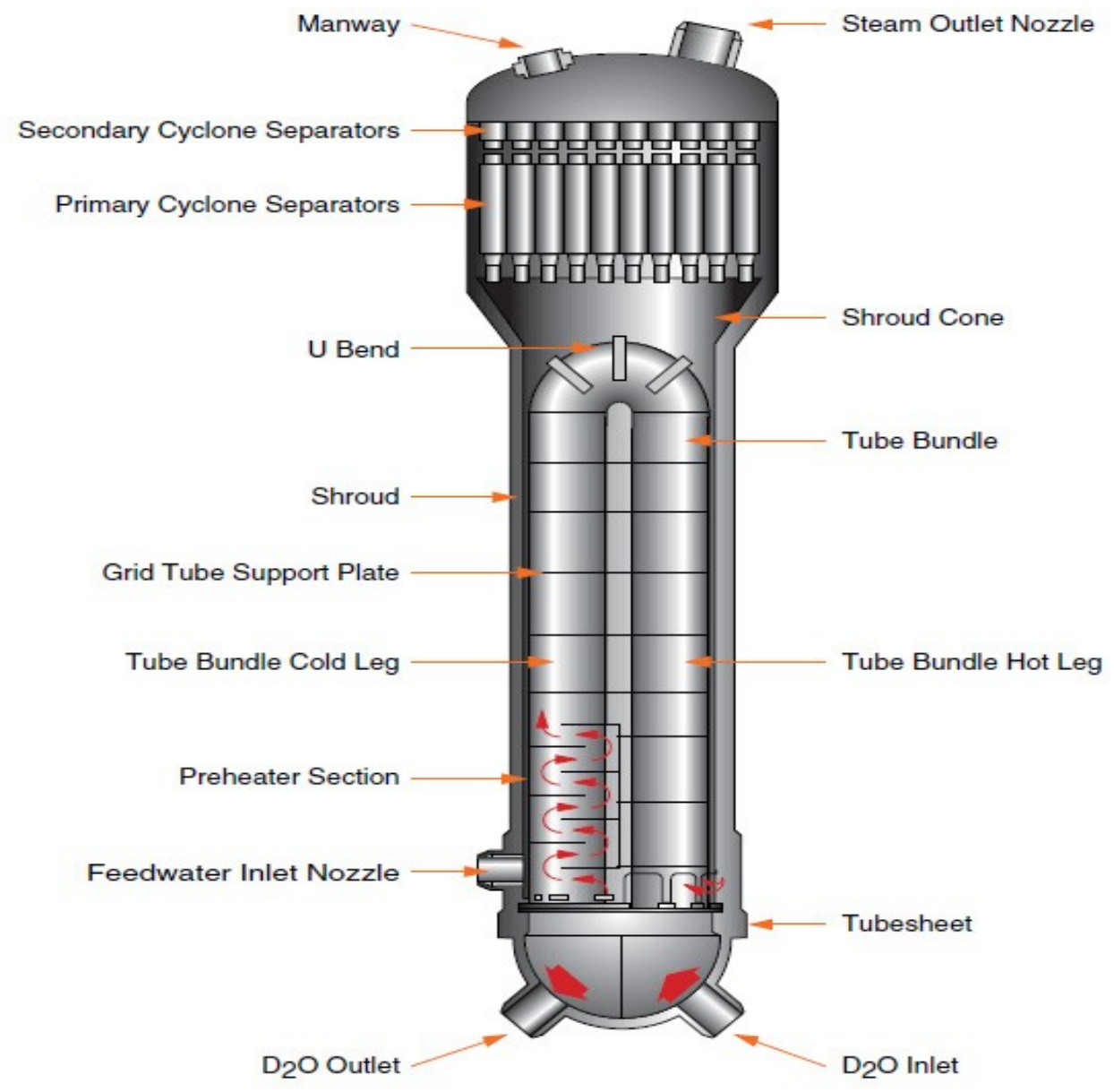

CANDU Steam Generator

9706782

Figure 1. 2 CANDU Steam Generator. Reproduced with permission CANTEACH [4]. 


\section{CHAPTER 2 NDT TECHNIQUES AND SG TUBE INSPECTION}

\subsection{Introduction}

NDT is the process of inspecting, testing or evaluating materials, components or assemblies for discontinuities or differences in characteristics without destroying the serviceability of the part or system. NDT is performed in a manner that does not affect the future usefulness of the object or material since it allows inspections without interfering with a product's final use and provides balance between quality control and cost-effectiveness.

NDT plays a critical role in assuring components and systems perform their function by implementing tests that locate and characterize material conditions and flaws. In addition, NDT inspections can prevent catastrophic situations such as reactors to fail, planes to crash and pipelines to burst.

In response to needs arising from various industries, a variety of NDT techniques have been developed such as radiography, magnetic particle, ultrasonic, liquid penetrant and electromagnetic testing methods [5].

Figure 2.1 shows a generic NDT system. The sensor couples the energy source into the test specimen. The actuator picks up the response of the field interaction and generates an output signal. The output signal is then processed using DSP algorithms, and passed through the 
classification analysis. The classification block performs defect characterization, which involves the estimation of defect dimensions, location, and shape.

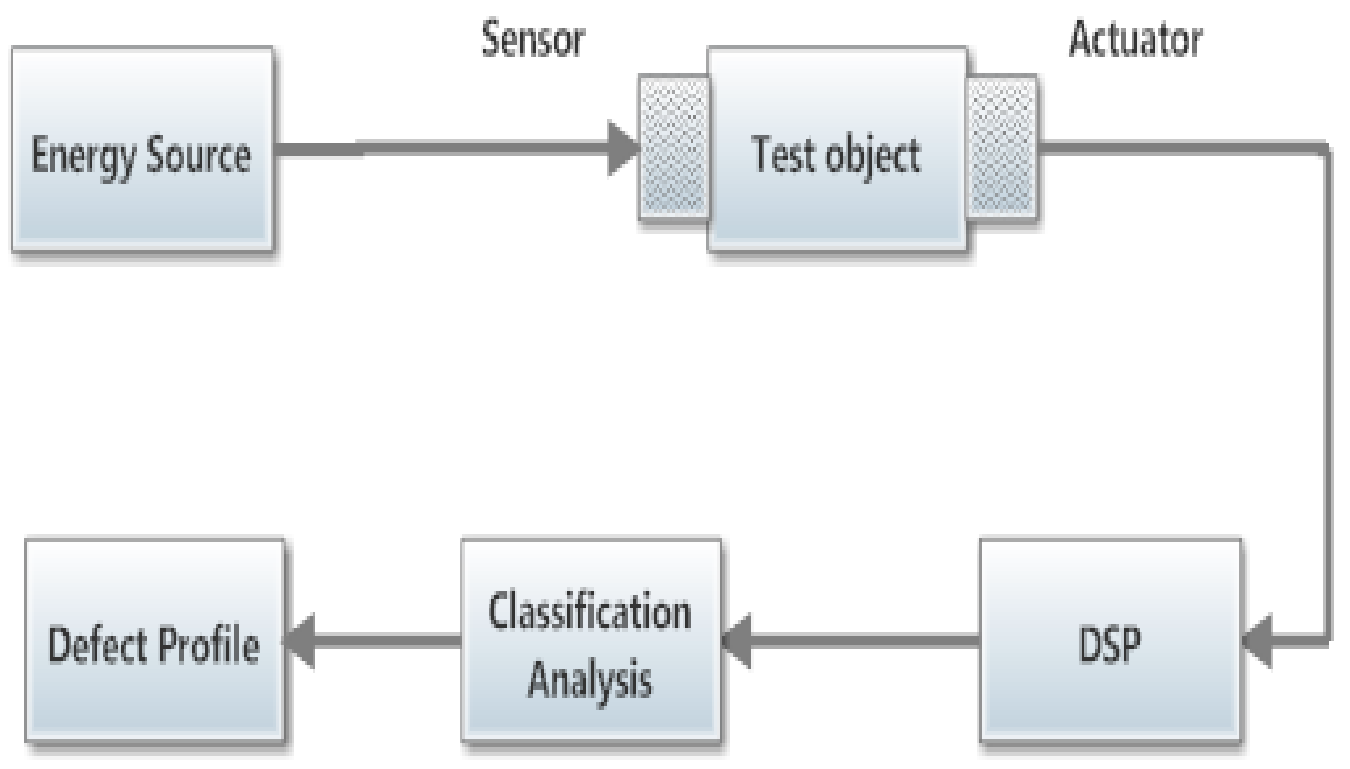

Figure 2. 1 Generic NDT System.

\subsection{NDT Methodologies}

There are eight common NDT methods that can be used to inspect components and make measurements. Some of the most commonly used NDT techniques are ultrasonic, radiographic and electromagnetic.

\subsubsection{Ultrasonic Testing Method}

The ultrasonic method is one of the oldest NDT techniques. In this method the probing source is ultrasound (high-frequency sound between $1 \mathrm{MHz}$ to $6 \mathrm{MHz}$ ). The ultrasonic method uses transmission of high-frequency sound waves into a material to detect imperfections or to locate changes in material properties. The most commonly used ultrasonic testing technique is 
pulse echo, where sound is introduced into a test object and reflections (echoes) are returned to a receiver from internal imperfections or from the part's geometrical surfaces. As shown in Figure 2.2 , high frequency sound waves are generated and sent into the material by the transducer. The received signal contains reflections due to defects. The time elapsed between the incident and the reflected signals are referred to as the time-of-flight (TOF). The amount of energy received and the TOF are analyzed to determine the presence of flaws and any changes in material thickness and properties. Ultrasonic inspection is very sensitive to both surface and subsurface discontinuities and very effective in measuring the depth of penetration. However, not all materials can be inspected, for example materials that are rough, irregular in shape or very small are difficult to inspect [7].
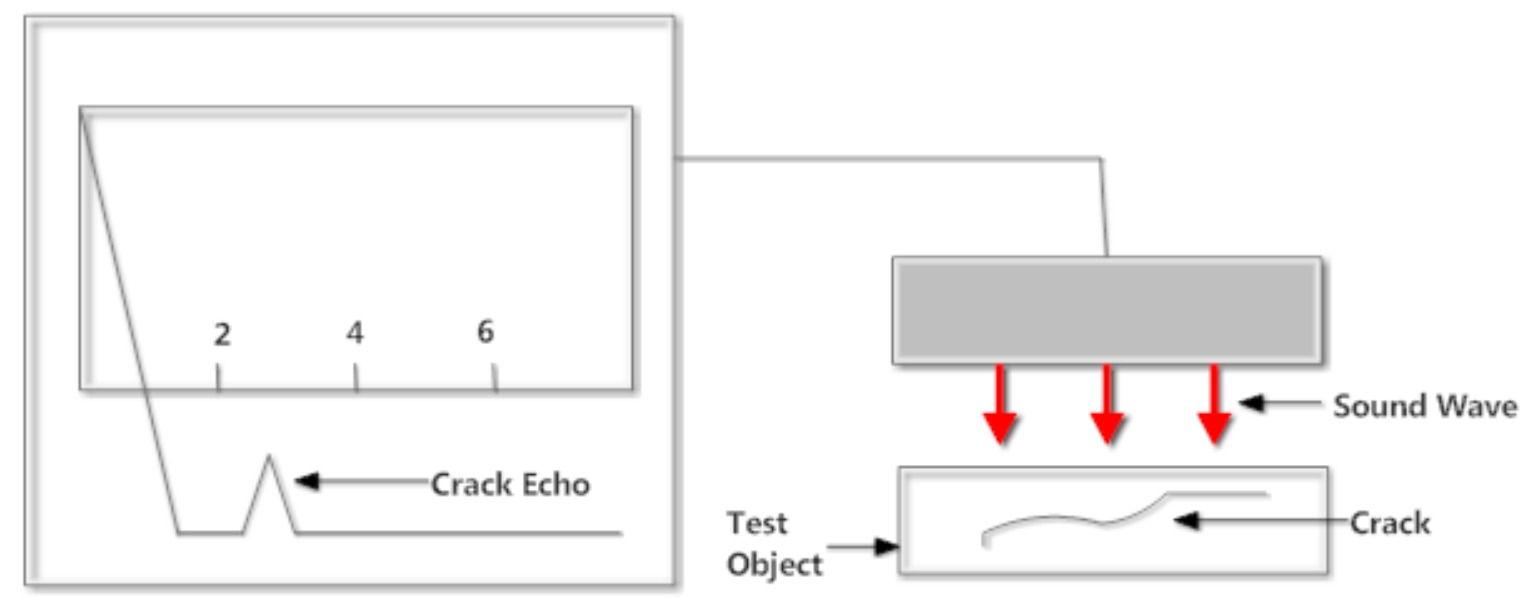

Figure 2. 2 Ultrasonic Testing Process.

\subsubsection{Radiographic Testing Method}

Radiographic methods involve the use of penetrating radiation to examine parts and products for imperfections. These are used to inspect almost any material for surface and subsurface defects. In addition, radiographic testing is used to locate and measure material's internal features and thickness. An X-ray or Gamma ray is usually used as the source of 
radiation. The intensity of the beam of energy transmitted through the object is reduced according to the thickness traversed by the beam and can be expressed as

$$
I_{t}=I_{0} e^{-\lambda t}
$$

where $t$ is the thickness of the material, $I_{0}$ and $I_{t}$ are the incident and transmitted energies respectively, and $\lambda$ is the linear absorption coefficient that depends on the material properties. As shown in Figure 2.3, the radiation passes through the test specimen, the radiation energy is recorded on a photographic film and analyzed to determine the location and internal features of the flaw. Radiographic inspection can be used to inspect all materials, but inspection of thick materials can be time consuming and relatively expensive. [8]

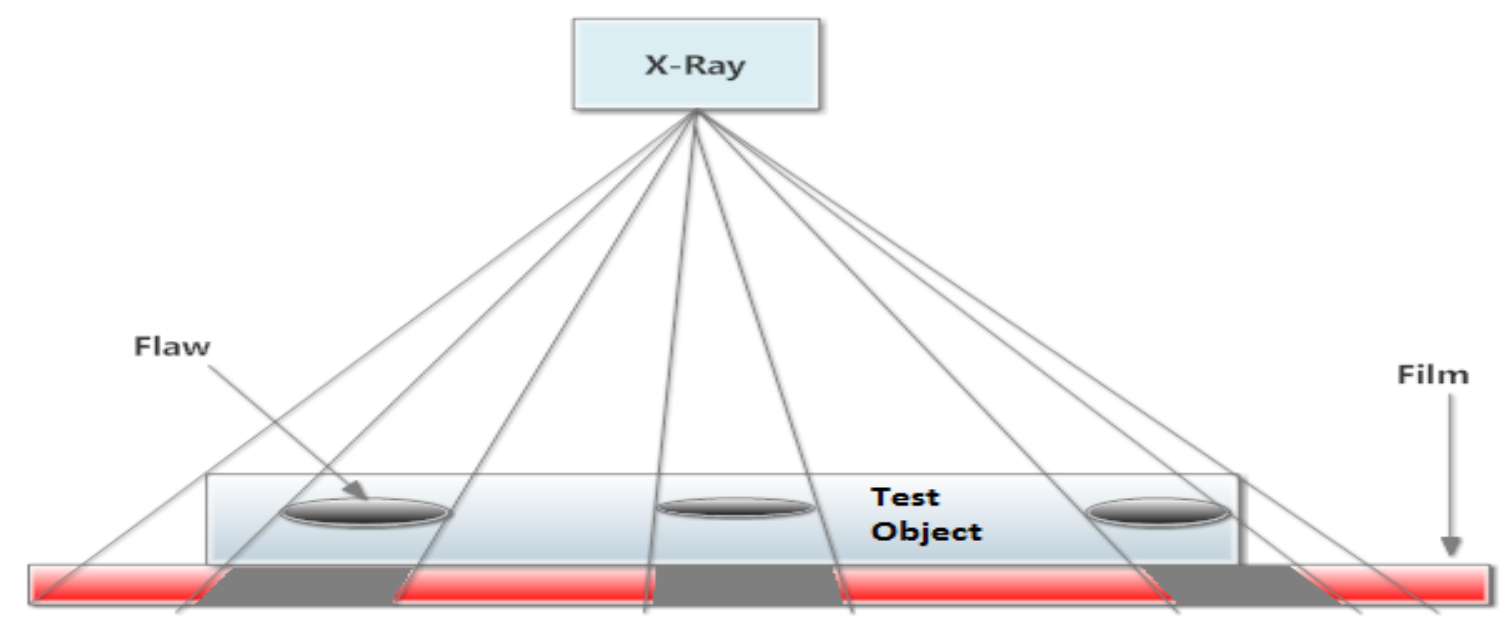

Figure 2. 3 Radiographic Testing Process.

\subsubsection{Electromagnetic Testing Method}

Electromagnetic NDT is the process of inducing electric currents or magnetic fields or both inside a test object and observing the electromagnetic response. Electromagnetic NDT has techniques which are used by many industries to characterize materials based on their electric and magnetic properties (conductivity and permeability). These techniques have many 
applications, such as stream generator tube testing, artificial heart valve implant and magnetic resonance imaging (MRI). ET is one of the most commonly used electromagnetic techniques. It employs a time-varying electromagnetic field as a source of excitation to evaluate the properties of the test material, detect discontinuities, measure variations in the geometry, and estimate dimensions of the test materials. ET methods are discussed in detail in section $2.3[9,10]$.

\subsection{ET Methods}

ET is used to test steam generators in nuclear power plant. The most common ET methods used are multi-frequency, remote field, swept frequency and pulsed ET.

\subsubsection{Eddy Current Theory}

Eddy currents are created through a process called electromagnetic induction. When alternating current is applied to a conductor, such as copper wire, a magnetic field develops in and around the conductor. This magnetic field expands as the alternating current rises to maximum and collapses as the current is reduced to zero. If another electrical conductor is brought into the close proximity to this changing magnetic field, a new current will be induced in this second conductor, this induced current is called eddy current. Eddy currents are closed loops of induced current circulation in the planes perpendicular to the magnetic flux. They get their name from "eddies" that are formed when a liquid or gas flows in a circular path around obstacles when conditions are right. Figure 2.4 shows eddy current magnetic field interactions [11].

As shown in Figure 2.4, an alternating electrical current is passed through a coil producing a magnetic field. When the coil is placed near a conductive test material, the changing 
magnetic field induces current flow in the test material. Eddy current is generated in closed loops and produces its own magnetic field that can be measured and used to find flaws and characterize conductivity, permeability, and dimensional features.

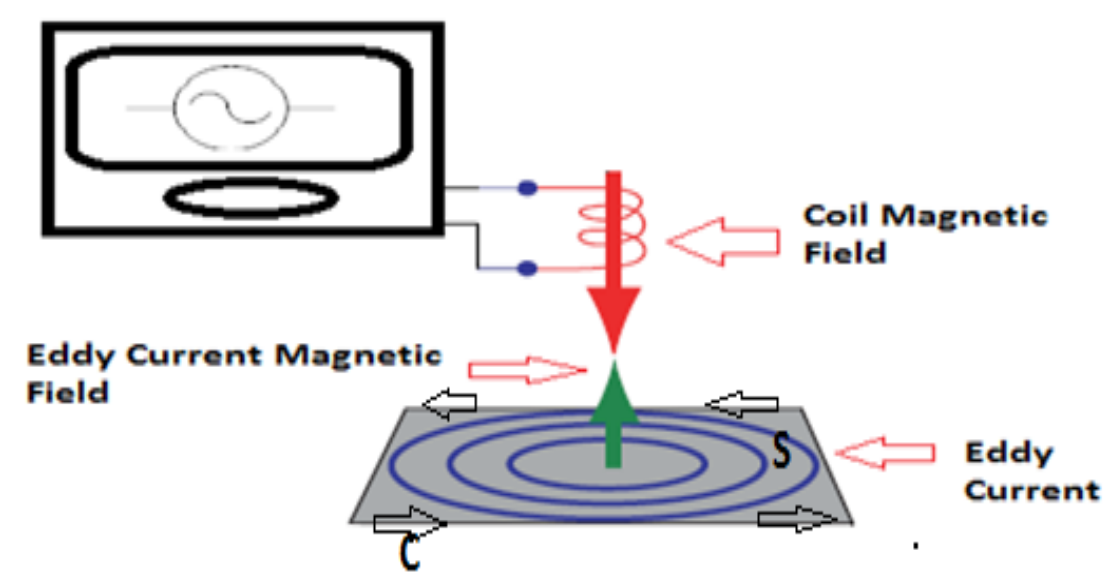

Figure 2. 4 Eddy Current Magnetic Fields Interactions.

Eddy current theory is based on the principles of electricity and magnetism, particularly the inductive properties of alternating current. Consider a simple coil excited by an alternating current. Since the coil is carrying an alternating current, an alternating magnetic field is set up in accordance with Maxwell-Ampere Law, where $\nabla$ is the curl operator, $\mathrm{H}$ is the magnetic field strength and $\mathrm{J}$ is the current density [11]

$$
\nabla \times H=J
$$

in the differential form and

$$
\oint_{C} H \cdot d l=\iint_{S} J \cdot d S
$$

in the integral form, where $\oint_{C}$ is the closed line integral around the closed curve of the conductive material $\mathrm{C}, d l$ is the differential line element around contour of the conductive 
material $C$, and $d S$ is the differential area element of the surface of the conductive material $S$, as shown in Figure 2.4, where the displacement currents have been neglected. Faraday's law states that if a time varying flux $\Phi$ is applied to a closed circuit, an electromotive force (emf) is induced. Emf $\varepsilon$ is the voltage developed by any source of electrical energy

$$
\varepsilon=-\frac{\partial \Phi}{\partial \mathrm{t}}
$$

If the coil is taken close to a conductive test specimen, an applied time varying flux causes an electromotive force to be induced in the specimen in accordance with MaxwellFaraday Law, where $E$ is the electrical field and $B$ is the magnetic field:

$$
\nabla \times \mathrm{E}=-\frac{\partial B}{\partial t}
$$

in the differential form and

$$
\oint_{C} E \cdot d l=-\frac{\partial}{\partial t} \iint_{S} B \cdot d S,
$$

in the integral form. The emf causes currents to flow in the specimen. These currents are called eddy-currents. The induced current, in turn, generates a field (induced or secondary field), whose direction is opposite to that of the primary field established by the coil, according to the Lenz's Law, as shown in Figure 2.4. This causes the net flux linking the coil to decrease.

The inductance of a coil is defined as the net flux linkages per Ampere, i.e,

$$
L=\frac{N \Phi}{I},
$$


where $L$ is the inductance, $N$ is the number of turns of the coil, $\Phi$ is the flux going through the area encircled by the coil, and $I$ is the source current. So the effective inductance of the coil decreases.

The inductance of the coil is affected by the distance between the coil and the test material, the variations of distance between the coil and the test material is called lift-off. Liftoff is the impedance change that occurs when there is variation in the distance between the inspection coil probe and the test piece. The lift-off variations can be caused by varying coating thicknesses, irregular sample surfaces or the operator's movements [13].

When a test coil is remote from any conductive material, impedance is at position of high inductive reactance and low resistance. The high value for the air point on the inductive reactance $(\mathrm{L})$ scale occurs because there is no secondary flux available to reduce primary flux and the only resistance $(\mathrm{R})$ detected is that of the coil wire, as shown in figure 2.5.

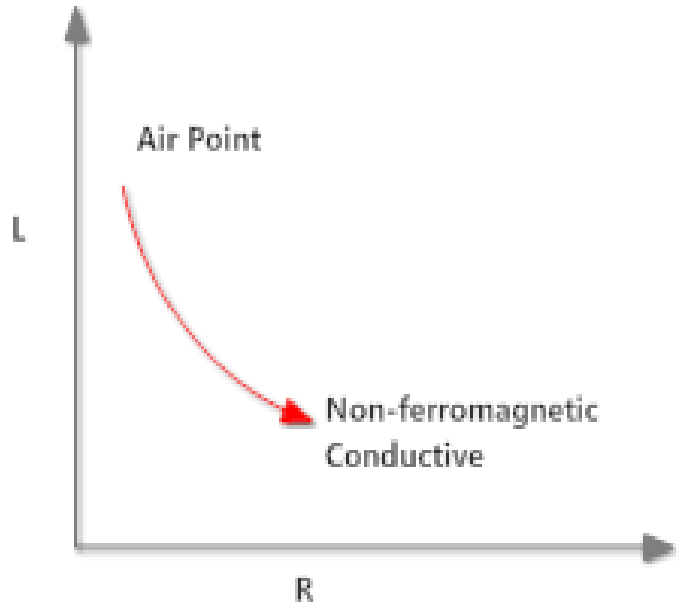

(a)

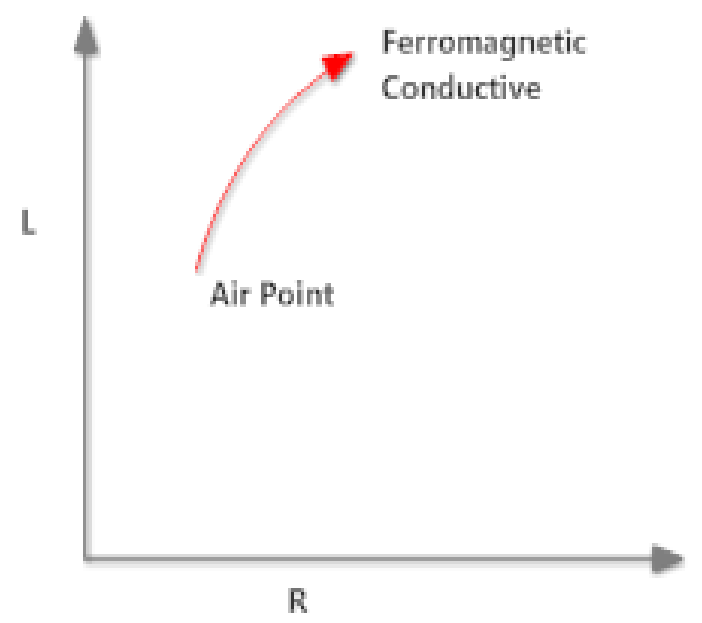

(b)

Figure 2. 5 Lift Off Curves a) Nonferromagnetic conductive material b) Ferromagnetic conductive material. 
As the coil approaches a nonferromagnetic conductive specimen ${ }^{7}$, the secondary flux cancels a portion of the primary flux, resulting in a decrease in the coil's inductive reactance. Simultaneously, the specimen acts as a resistive load on the coil and the impedance point advances along the resistance scale, as shown in Figure 2.5a. However, if the coil approaches a specimen that is both ferromagnetic and conductive, the specimen's flux is added to the coil's flux and the impedance point moves up both the inductive reactance and resistance scale, as shown in Figure 2.5b [13].

The eddy-currents induced in a test material decay rapidly as the depth of the test material increases. This is called the skin depth phenomenon. The current density is a maximum at the material surface and decreases exponentially with depth. Thus, in the case of thick specimens with high conductivity, eddy-currents are largely confined to the outer skin of the test material and test sensitivity to subsurface defects decreases rapidly with depth. The term skin depth $(\delta)$, also called standard depth of penetration, as shown in Figure 2.6, is defined as the depth at which eddy-current density has decreased to 1/e (e is Euler's number) of its surface value. The skin depth for the case where a current sheet ${ }^{8}$ is present at the surface of a half plane is given by [13]:

$$
\delta=\frac{1}{\sqrt{\pi \mu f \sigma}}
$$

where $f$ is the excitation frequency of the circuit, $\mu$ is the magnetic permeability of the target material, and $\sigma$ is the electrical conductivity of the target material. The skin depth is often used as a guideline to select the excitation frequency for a given test specimen, Figure 2.6 shows the skin depth phenomenon [6].

\footnotetext{
${ }^{7}$ Specimen is a test material

${ }^{8}$ Current sheet is an electric current that is confined to a surface, rather than being spread through a volume of space
} 


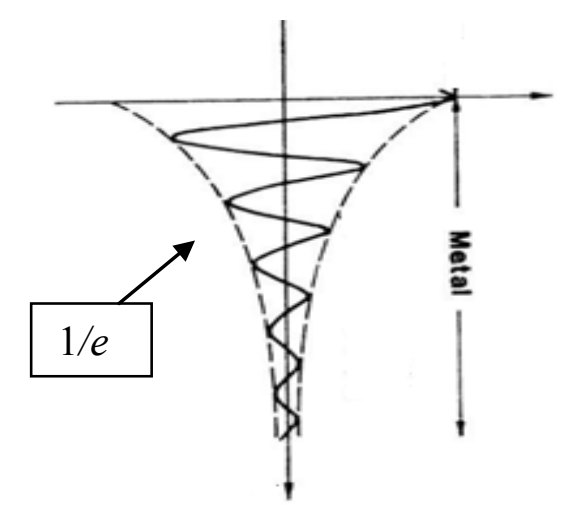

Figure 2. 6 Skin depth phenomenon.

\subsubsection{Multi-Frequency ET}

Multi-frequency techniques are widely used in non-destructive ET and overcome the single frequency limitation. Multi-frequency techniques expand the capabilities of singlefrequency testing and save time since they allow simultaneous tests. This provides data that are analyzed using multi-frequency mixing or multi-parameter techniques which allows the effect of extraneous discontinuities such as support plates, to be suppressed [6].

Each testing frequency is sensitive to a certain type of discontinuity. Low frequencies have a large skin depth and hence give clear signals from support structures that are located away from the coil and are used to determine the location of the probe along the tube. At high frequencies, eddy-currents have a much smaller skin depth and for defects on the outside of the tube, the depth can be estimated from the phase of the eddy-current signal. Because of the different skin depths at different frequencies, the relationship between signals from defects and support features changes with frequency. Consequently, it is possible to combine the signals from two different frequencies so as to subtract out a support feature but leave the signal from defect relatively intact. In effect, this means that multi-frequency response signals contain more 
information that can be analyzed to extract relevant features. In summary, multi-frequency techniques have the following advantages [6]:

- Collects data at several test frequencies simultaneously (this decreases the in-service inspection time and human exposure to radiation),

- Allows separation of discontinuities that give similar signals at one frequency,

- Improves sensitivity to different types of discontinuities,

- Improves the detection, interpretation and sizing of defects even in the presence of artefacts that complicate the analysis procedure.

\subsubsection{Remote Field Eddy Current Method}

The remote field eddy-current (RFEC) techniques are considered highly sensitive to material discontinuities at large depths (inner or outer diameter in the tube wall). In detecting residual stress in infrastructures, which is becoming more and more of concern, the RFEC techniques are more useful than conventional ET techniques.

The RFEC techniques were first introduced for pipe and tube inspection. A coil excited by an alternating current is placed in a pipe; the energy diffuses along two different paths: direct path and indirect path. It has been shown that the energy diffusing via the direct path attenuates very rapidly, because it is restricted by eddy-current in the pipe wall. When a pickup coil is located at a certain distance away from the excitation coil, the received signal is primarily due to the energy diffusing via the indirect path. This portion of the energy passes the pipe wall twice before arriving at the pickup coil. So the received signal is closely related to the thickness, conductivity, permeability, wall conditions. Its phase is linearly proportional to the wall 
thickness. Recently, the RFEC technique has been successfully applied to detect compressive residual stress ${ }^{9}$ in carbon steel specimens of flat geometries [12].

\subsubsection{Swept Frequency Eddy Current Method}

The swept frequency eddy current method involves collecting eddy current data at a broad range of frequencies. The testing process involves using an impedance analyzer, which can be configured to automatically make measurements over a range of frequencies. The swept frequency method is used to measure the thickness of conductive coatings on conductive base metal, distinguishing between flaws in surface coatings and flaws in the base metal. An example application would be the lap splice ${ }^{10}$ of a commercial aircraft.

The advantages of swept frequency testing is that depth information can be obtained since eddy current depth of penetration varies as a function of frequency and makes it possible to tell if cracking was occurring on the outer skin, the inner skin or a double layer. On the other hand, the swept-frequency technique is difficult to implement and time-consuming [13].

\subsubsection{Pulsed Eddy Current Method}

The pulsed eddy current (PEC) technique uses a step function voltage to excite the probe $^{11}$. The improvement over the conventional eddy current method is that the pulsed eddy current technique uses a step function voltage that contains a range of frequencies. As a result, the eddy current measurements at several different frequencies can be done with just a single step. In addition, material depth information can be obtained at once at different frequencies, since the depth of penetration is dependent on the frequency of excitation. The measured

\footnotetext{
${ }^{9}$ Compressive residual are stresses that remain in a solid material after the original cause of the stresses has been removed.

${ }^{10}$ Lap splice is a method of joining two structural members end to end.

${ }^{11}$ There are three common probes used in testing which are bobbin, pancake and X-probe.
} 
response of a PEC inspection is a waveform, similar to an ultrasonic scan, from which features can be extracted to characterize flaws or perform thickness measurements. A reference signal is usually collected, to which all other signals are compared in order to detect flaws, conductivity, and dimensional changes [14].

The pulsed eddy current inspections can be carried out through any material. In addition, it has very useful characteristic that enables high temperature testing or offshore well inspections.

\subsection{ET Apparatus}

Eddy current instruments can be designed in a large variety of configurations. Instruments are commonly classified by the type of display used to present the data. The common display types are analog meter, digital readout, impedance plane and signal amplitude versus time. Some instruments are capable of presenting data in several display formats [15].

The most basic ET instrument consists of an alternating current source, a coil of wire connected to this source, and a voltmeter to measure the voltage change across the coil. Figure 2.7 presents a block diagram of a basic eddy current system apparatus. It includes a single frequency oscillator, amplifiers, quadrature amplitude demodulator, and impedance adaptation circuit. The oscillator is used to tune to the testing frequency. The impedance adaptation is a bridge circuit which is used to balance the measured voltage and to provide a reference signal. Any change in the condition of the coil will result in an unbalanced bridge which generates a defect signal. The defect signal is demodulated using the quadrature amplitude demodulator which extracts the defect signal and outputs the signals on $\mathrm{X}$-axis and $\mathrm{Y}$-axis. Each component represents the real and imaginary parts of the impedance respectively [11].

Factors that influence the eddy current field, and coil impedance, are: 
- The separation between the coil and specimen surface, called lift-off

- The electrical conductivity of the specimen

- The magnetic permeability of the specimen

- The frequency of the alternating current inducing the eddy current field

- The design of the eddy current probe

- Geometric factors

- Discontinuities, such as cracks, corrosion and pitting

Improving the detection and characterization of flaws requires a sophisticated design of DSP procedures to compensate for these effects. The elimination of undesired responses and extraction and interpretation of relevant information forms the basis of considerable research activity in eddy current inspection.

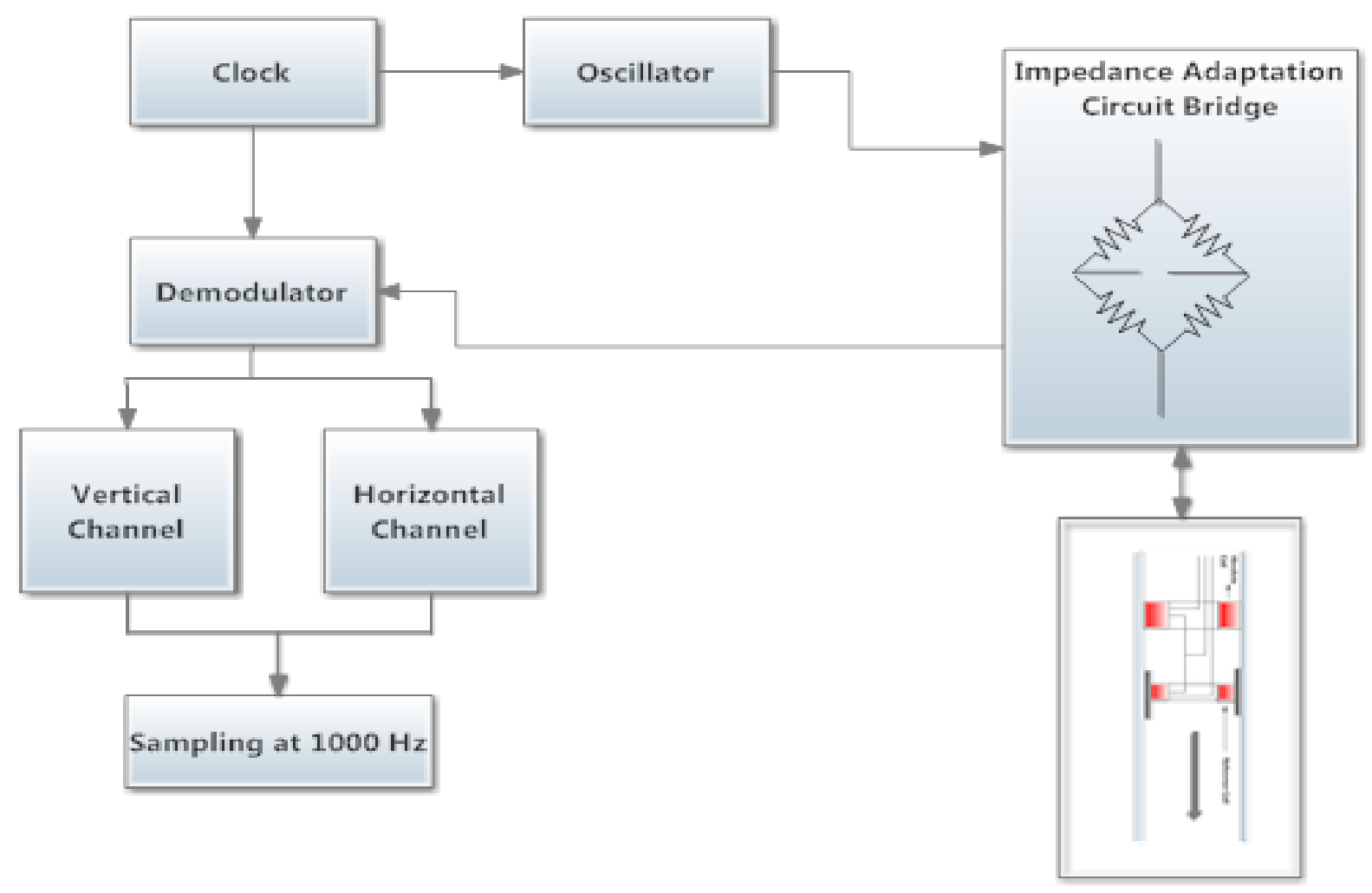

Figure 2. 7 Eddy Current Basic Testing Apparatus. 


\subsection{Bobbin Probe}

Three major types of multi-frequency eddy current probes are used in practice for SG tube inspection. The most popular probe type is the bobbin coil probe, which consists of two identical coils connected in a differential mode ${ }^{12}$ and excited at multiple frequencies. The advantage of this probe is that its signal is insensitive to various anomalous effects, such as temperature variations and gradual variations in the inspected tube's electrical conductivity and diameter. This probe is very sensitive to abrupt anomalies, such as pitting, corrosion and fretting ${ }^{13}$ wear. Although the bobbin coil probe is the most widely used probe, it has limitations in its ability to detect degradation in all regions of the tube, the ability to accurately size and characterize degradation and the probe's insensitivity to circumferentially oriented flaws. As a result of these limitations, the bobbin coil probe is mainly used for the initial detection of possible degradation to quickly determine those areas of the tube requiring additional inspection with other types of probes that have improved ability to size and characterize degradation, such as rotating $\operatorname{probes}^{14}[6]$.

\subsection{Data Collection}

The data records used in this thesis were collected by inspectors of SG tubes in nuclear power plants. The eddy current measurements were collected using an absolute bobbin probe with conventional eddy current system, as shown in Figure 2.7.

The eddy current data records are demodulated using a quadrature amplitude demodulator with center frequency equal to the testing frequency. The output of the eddy current

\footnotetext{
${ }^{12}$ Differential mode is a method of transmitting information electrically with two complementary signals sent on paired wires.

${ }^{13}$ Fretting wear is the repeated cyclical rubbing between two surfaces.

${ }^{14}$ A rotating probe consists of three different types of coils rotating inside the tube at high speeds and traveling in the axial direction.
} 
instrument has two components the real and imaginary parts which are calibrated and sampled at $1000 \mathrm{~Hz}$. Figure 2.8 shows a differential bobbin probe scanning the tube.

Commonly, the lowest test frequency is $30 \mathrm{kHz}$ which has low sensitivity to flaw signals, but high sensitivity to signals from support plates. The higher frequencies used for tube inspection are 120, 240 and $480 \mathrm{kHz}$. At these frequencies, the flaw signals are more pronounced relative to the tube support plate signals than at $30 \mathrm{kHz}$. A data record from TUBE 1 showing the horizontal (real) and vertical (imaginary) channels at $240 \mathrm{kHz}$ are shown in Figure 2.9. Both horizontal and vertical channels convey the same information in terms of support plates and peak locations. We notice that the data record is very noisy and exhibits high peaks at the beginning and ending, this is due to the probe entering and exiting the tube.

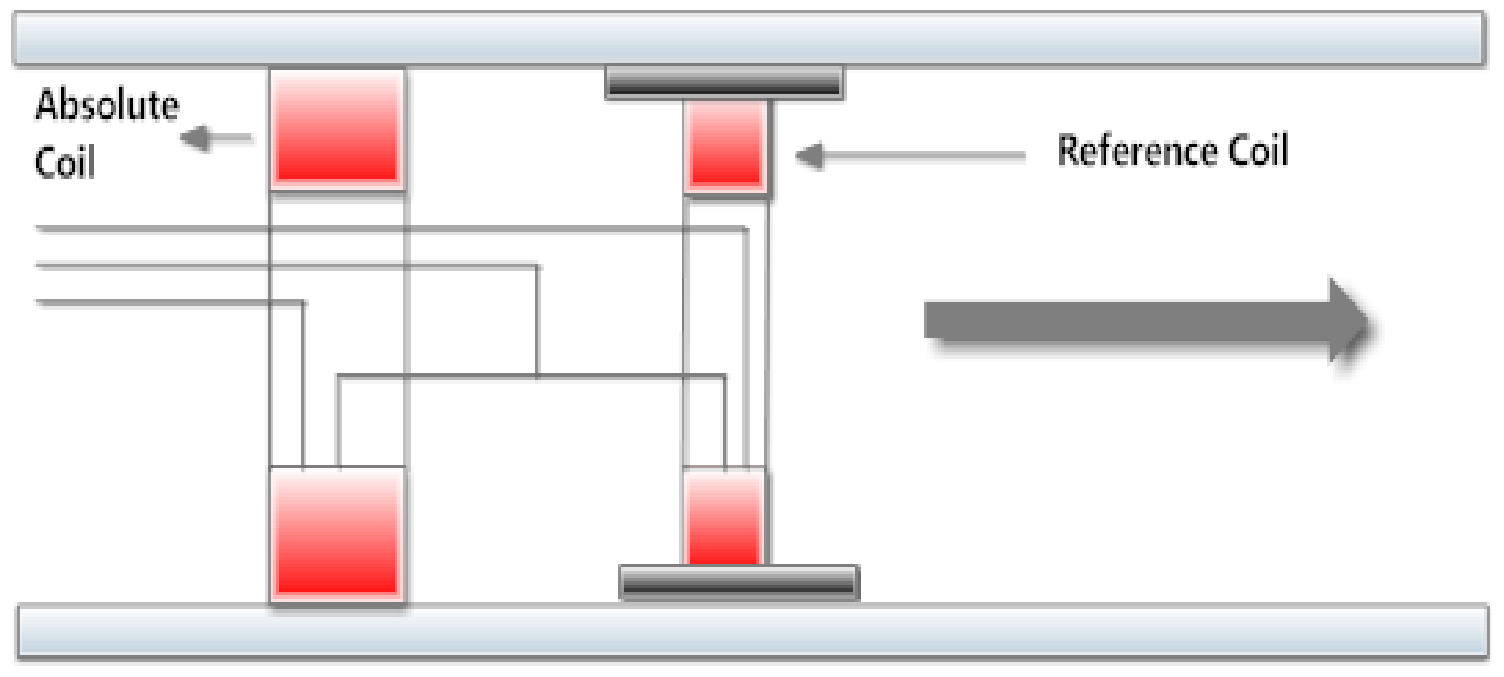

Figure 2. 8 Differential Bobbin Probe Scanning a Heat Exchanger Tube.

The data record is corrupted by many sources of distortions. These sources are a combination of deterministic and random noise. The flaw indication signal in the data record exhibits a peak. These peaks are used to determine what kind of flaw and the location of the 
flaw in the SG. Flaw signals from TUBE 1 at different frequencies are shown in Figure 2.10. We notice that the flaw signal has its maximum peak at $240 \mathrm{kHz}$.
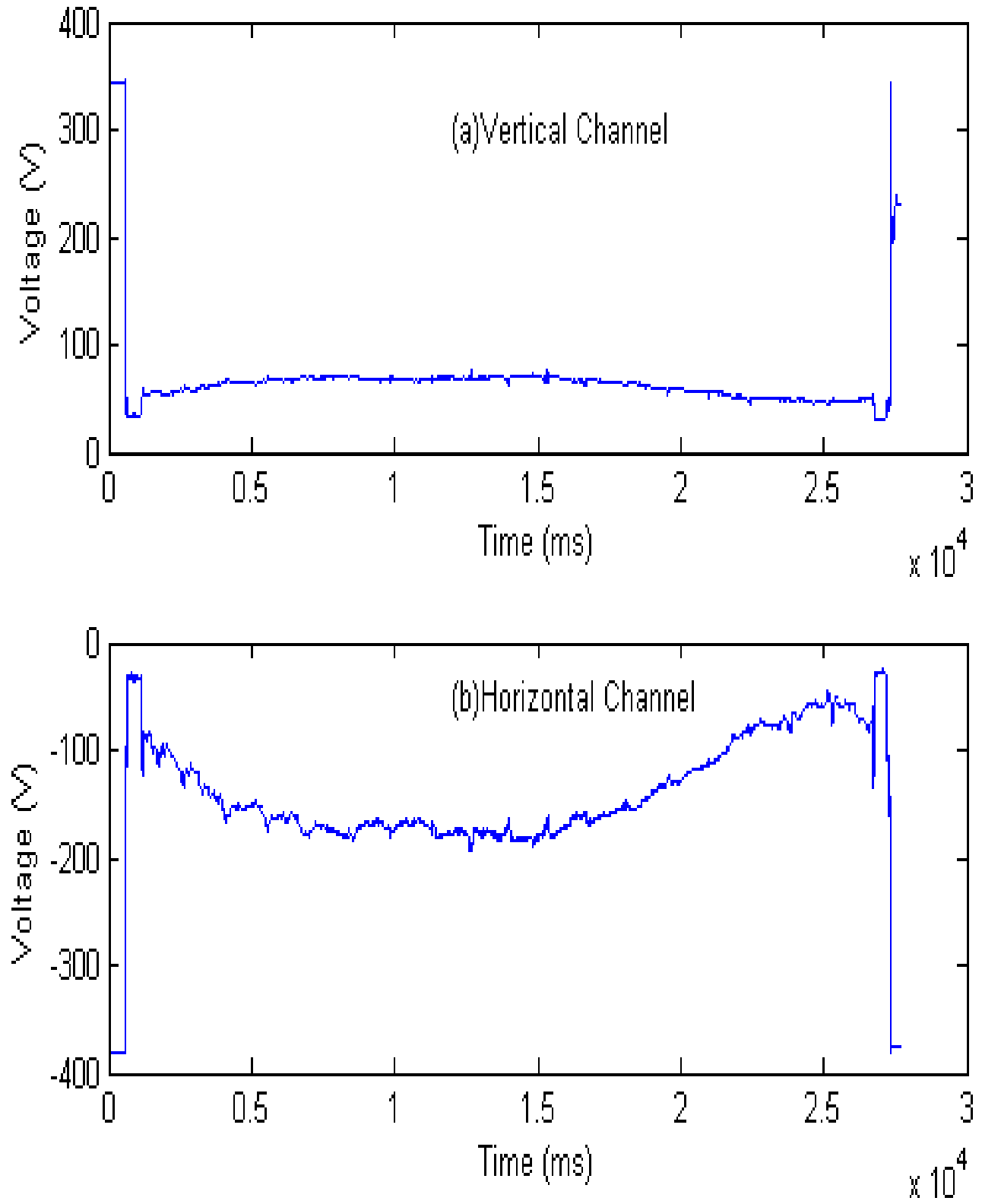

Figure 2. 9 Typical Eddy Current Data Record for a) Vertical Channel and b) Horizontal Channel for $240 \mathrm{kHz}$ (TUBE 1). 


\subsection{Sources of Signal Distortion}

For inspection applications, signal essentially refers to the probe response to any consequential indication associated with various forms of degradations and volumetric crack-like signals. For SG tubing, this definition covers a wide range of electronic and physical sources that may be located either internal or external to the tube. These sources can be classified as deterministic or random distortion [15].

\subsubsection{Deterministic Distortion}

The sources of deterministic distortions include:
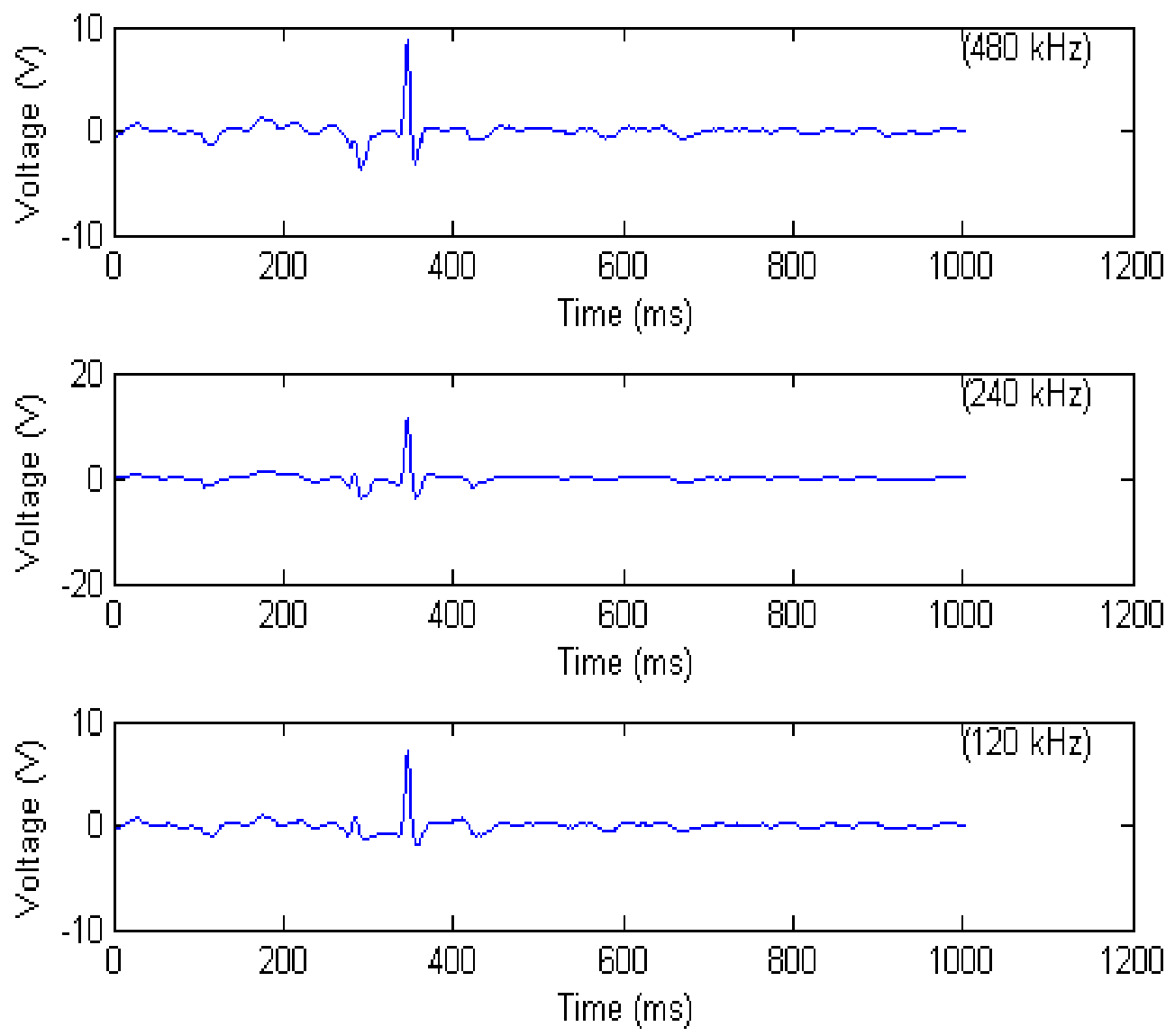

Figure 2. 10 Flaw Signals in Eddy Current Record at 480, 240 And 120 kHz (TUBE 1). 
- Support structures have mixed designs and are made of materials that produce different characteristic responses. For instance, the eddy current probe response from drilled support structures are easier to analyze than that from broached and lattice supports. This is due to the difference in uniformity in the geometry of the support structure itself. Support plate responses from TUBE one at different frequencies is shown in Figure 2.11.

- Corrosion products, which can gather on the outer or inner surface of the tube and can vary widely in terms of conductivity and permeability of the material.

- Variations in tube dimension typically cause the most evident distortion of ET signals. Such changes include reduction of the tube diameter due to denting and ovalization of the tube at small radius U-bends.

- An increase in probe lift-off produces a drop in the flaw response and is directly connected with the degree of coupling between the probe and the tube.

- Probe wobble demonstrates itself as a low-frequency periodic response that is due to a change in the alignment of the probe with respect to the tube axis, uneven wearing of the centering device, probe guide, coil housing or tube pliger ${ }^{15}$. Probe wobble response from TUBE one is shown in Figure2.12.

\subsubsection{Random Distortion}

The sources of random distortions include [15]:

- Random noise can be generated by spatial variability of the electrical conductivity and magnetic permeability of the tubing. Depending on the type of variability, both the

\footnotetext{
${ }^{15}$ Tube pliger is variation in tube inside diameter caused by manufacturing process
} 
resistive and reactive components of the probe impedance could be affected. The harsh environment of the SG is the main source of this type of noise.

- Electronic or system noise can originate either from the eddy current test equipment or by interference from external sources. Electronic noise can exhibit itself either as a gradual signal drift caused by temperature fluctuation in the circuit components and probe. In addition, electromagnetic or electrical interference can be caused by power lines, cable connections, and nearby electrical equipment [15].
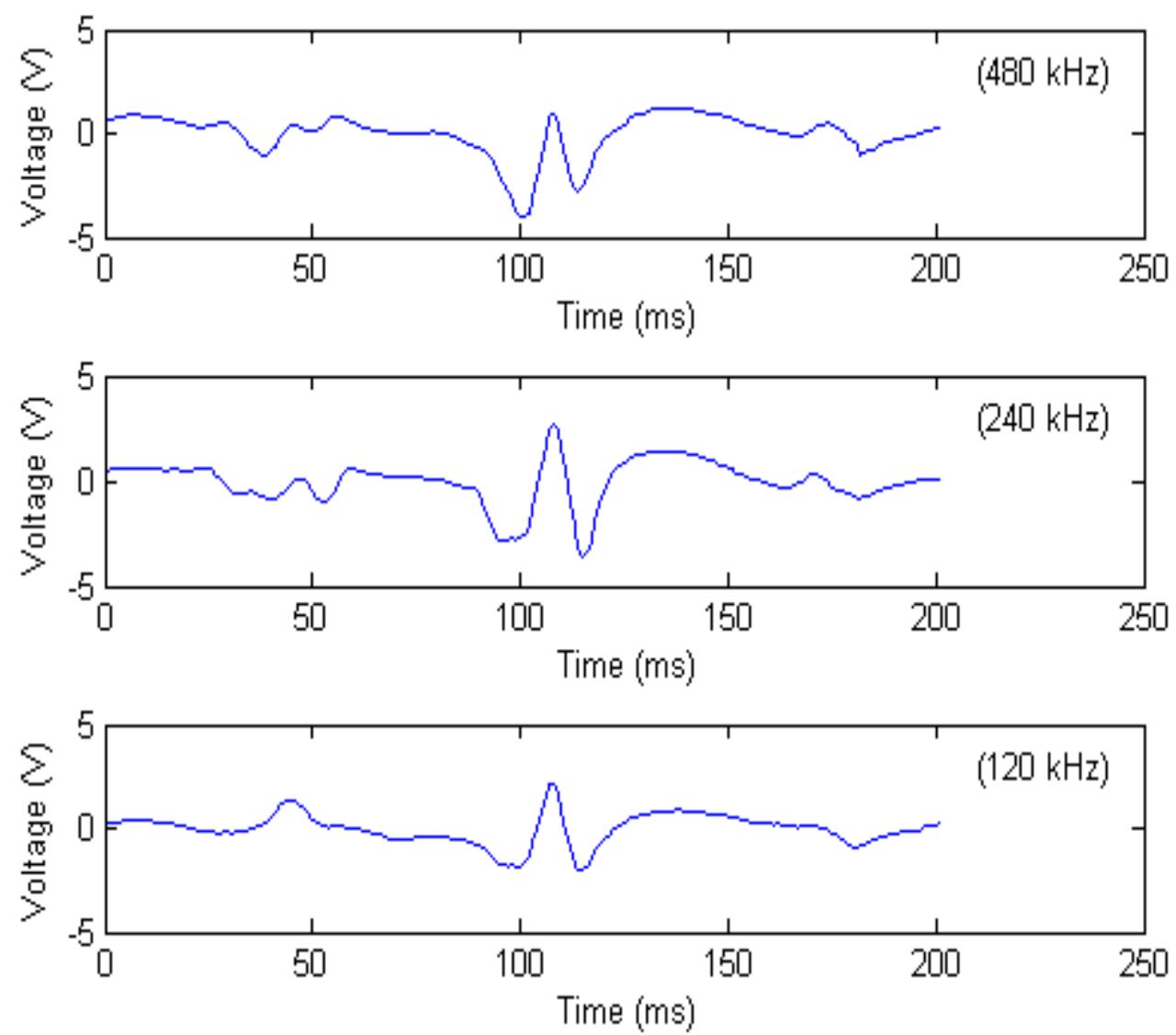

Figure 2. 11 Support Plate Responses at 480, 240 and $120 \mathrm{KHz}$ (TUBE 1). 


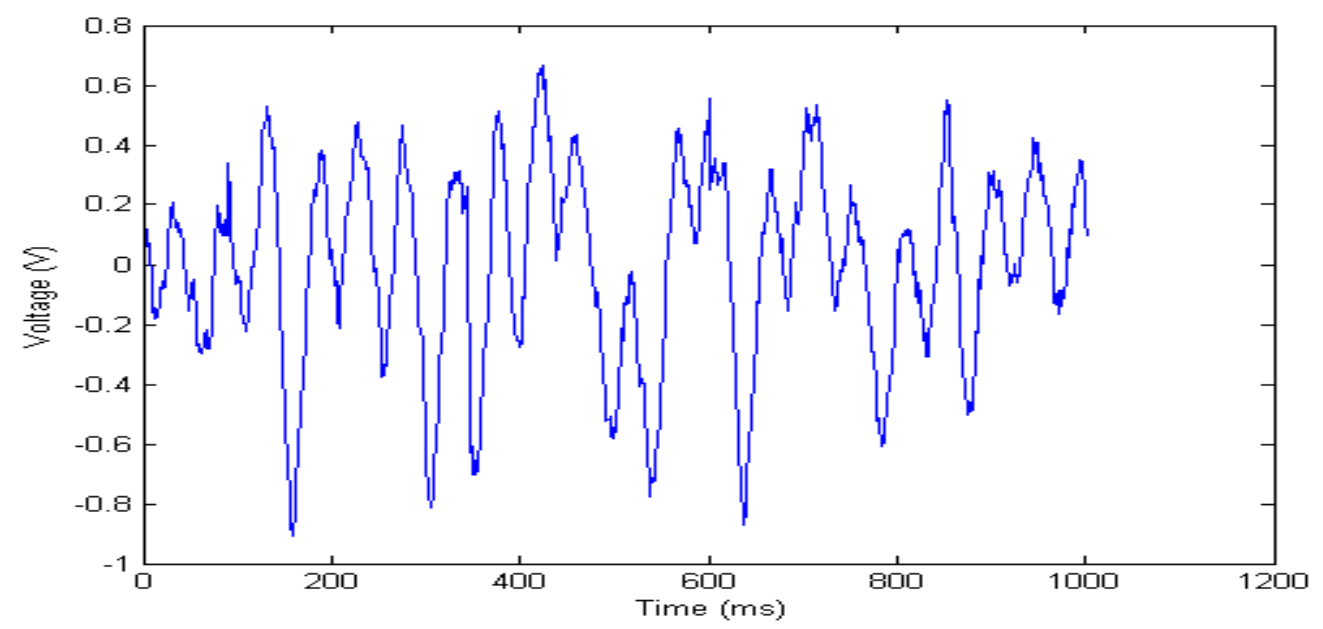

Figure 2. 12 Probe Wobble Response (TUBE 1)

\subsection{Research Objective}

Although it is relatively easy to understand the basic eddy current probe-flaw interaction, real world analysis of SG tube eddy current data is difficult due to noise and many unwanted indications that cause significant distortion in the flaw signal. Automatic flaw detection systems for bobbin coil eddy current data have been well studied by many researchers. These studies mainly focus on the relationship between flaw characteristics and the shape and orientation of the corresponding Lissajous pattern of the eddy current signal and attempt to mimic the decision process of a human expert.

Any undesirable probe response from any extraneous source that complicates the detection and interpretation of flaw signals would be considered as noise. These unwanted signals can be classified broadly as either random or deterministic. Deterministic noise exhibits a predictable spatial (i.e., as a function of position) and frequency distribution. Random noise, on the other hand exhibits incoherent spatial and frequency distribution [15]. 
Low-frequency cyclic sinusoidal noise originates from periodic variations in the material properties or dimensions caused by manufacturing processes or the inspection system such as probe wobble which can be caused by changes in the alignment of the probe with respect to the tube axis, problem with the probe positioning mechanism, uneven wearing of the centering device, probe guide or coil housing.

The objective of this thesis is to estimate and remove the low frequency sinusoidal responses by employing DSP algorithms. The process uses a multi-step method to accomplish this task. The first step involves preprocessing stages to remove trends and biases, compensate for the magnitude change, clean up the artefacts and segment the data record. The second step involves estimating the low-frequency sinusoidal parameters and removing the sinusoidal interference. Figure 2.13 shows a block diagram for the whole process.

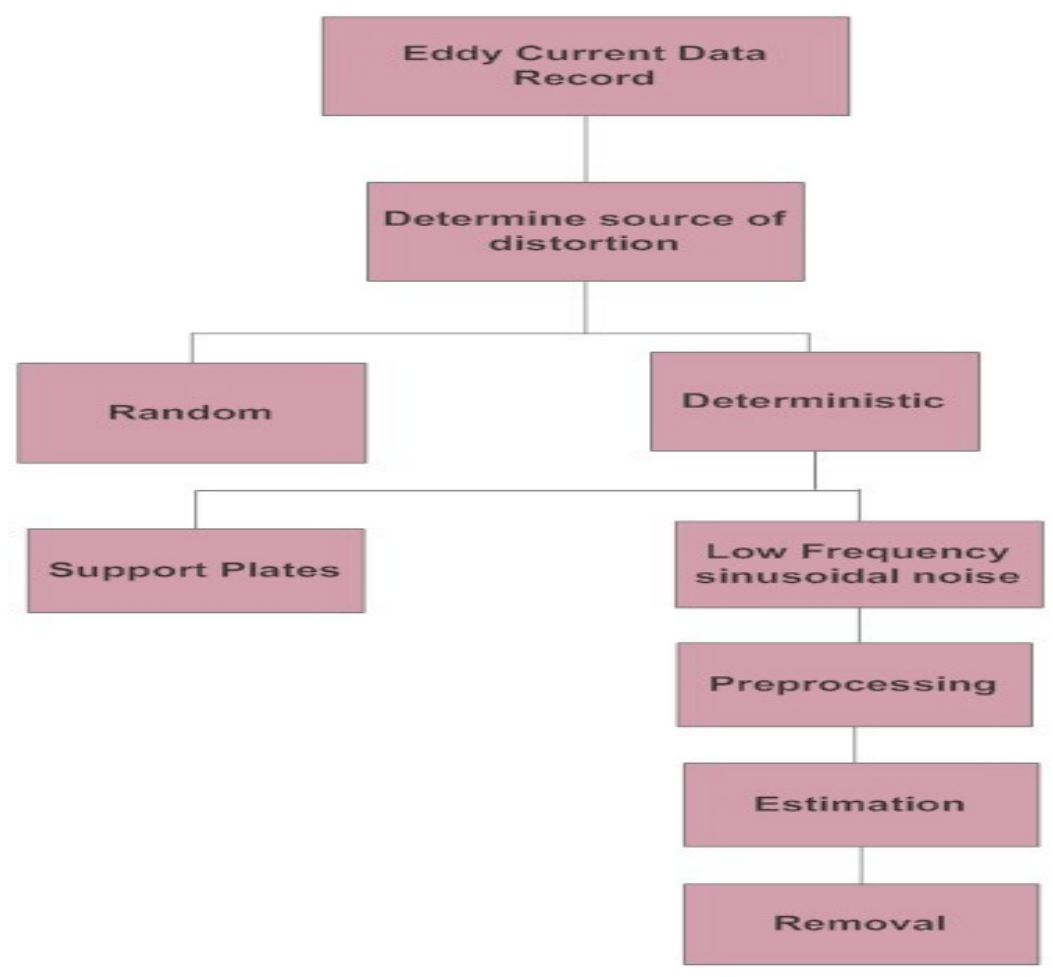

Figure 2. 13 Block Diagram of the Noise Removal Process 


\section{CHAPTER 3 LITERATURE REVIEW}

\subsection{Introduction}

The analysis of eddy current data collected from nuclear SGs is very important and very labor intensive. To aid the analysis team many researchers have been working on developing automatic data analysis systems to detect and characterize the flaw signals. A Dynamic thresholding algorithm based on Neyman-Pearson (N-P) hypothesis testing was proposed for use in an automatic data analysis system [16]. This technique reduces the number of data points that need to be further analyzed and detects almost all flaw signals. In addition, an adaptive Wiener filtering (AWF) technique is proposed to de-noise the data record and significantly enhance the defect signals [18].

\subsection{Dynamic Thresholding for Automated Analysis of}

\section{Bobbin Probe Eddy Current Data}

The automatic procedure for analyzing multi-frequency eddy current data records employs dynamic thresholding. Dynamic thresholding is based on the N-P detector which computes a dynamic or adaptive threshold value that separates data points into one of two classes, noise and potential defect indications. The data must be preprocessed before applying the N-P detector. The preprocessing step filters the data to remove any biases and trends that may be present using a zero-phase high-pass filter. Zero-phase filtering is used to ensure that no 
shifts in the signal are introduced and the cut-off frequency for the HP filter was chosen to be around $50 \mathrm{~Hz}^{16}[16]$.

\subsubsection{N-P Detector Theory}

An N-P detector maximizes the probability of detecting a signal (in presence of noise) for a given probability of false alarm (PFA). This objective can be accomplished by using a hypothesis testing approach as indicated in $[16,17]$. The hypotheses are given by

$H_{0}: X(n)=W(n)$

$H_{1}: X(n)=S(n)+W(n), \quad n=1,2, \ldots \ldots, N$

where, $X(n)$ is the measured signal, $S(n)$ is the defect signal, $\mathrm{W}(\mathrm{n})$ is the additive noise and $\mathrm{N}$ is length of the measured signal. The $N-P$ detector uses the log likelihood ratio to compute a test statistic that is used for threshold computation. The log-likelihood ratio is specified as

$T(X)=\log \left[\frac{P\left[X / H_{1}\right]}{P\left[X / H_{0}\right]}\right]$

where, $P\left[X \mid H_{1}\right]$ is the probability density function (PDF) of $X$ given $H_{1}$ and $P\left[X \mid H_{0}\right]$ is the PDF of $X$ given $H_{0}$. Assuming that the noise is additive white Gaussian, with zero mean and variance $\sigma_{\omega}^{2}$, and the signal is distributed as a multivariate Gaussian, with covariance $\mathrm{NxN}$ matrix $C_{s}$ and mean $1 \mathrm{xN}$ vector $\mu_{s}$ :

$$
\begin{aligned}
& W \sim \mathrm{N}\left(0, \sigma_{\omega}^{2}\right) \\
& S \sim \mathrm{N}\left(\mu_{\mathrm{s}}, \mathrm{C}_{\mathrm{s}}\right)
\end{aligned}
$$

The test statistic is scalar value which can then be formulated using equations (2), (3a) and (3b):

$T(X)=X^{T}\left(C_{s}+\sigma_{\omega}^{2} I\right)^{-1} \mu_{s}+\frac{1}{2 \sigma_{\omega}^{2}} X^{T}\left[C_{s}\left(C_{s}+\sigma_{\omega}^{2} I\right)^{-1}\right] X$

\footnotetext{
${ }^{16} 50 \mathrm{~Hz}$ low frequency noise assumed by the author
} 
where I is the $\mathrm{NxN}$ identity matrix

This test statistic is then compared to a threshold $\lambda$ to determine which of the two hypotheses is true:

If $T(X) \leq \lambda, H_{0}$ is true

If $T(X)>\lambda, H_{1}$ is true

The threshold $\lambda$ is obtained using a constraint on the probability of false alarm (PFA)

$P F A=\int_{\lambda}^{\infty} P\left[T(X) / H_{0}\right] d T$

Equation (6) uses $P\left[T(X) / H_{0}\right]$ which is the PDF of $\mathrm{T}(\mathrm{X})$ given $H_{0}$, to compute the threshold $\lambda$ for a given value of PFA. Based on the assumption that the signal and noise are both zero-mean and white, then

$T(X)=\frac{\sigma_{s}^{2}}{\sigma_{\omega}^{2}\left(\sigma_{s}^{2}+\sigma_{\omega}^{2}\right)} X^{T} X$

All data points whose test statistics values are greater than $\lambda$ are marked as potential defect signal points while all other data points are discarded as noise points.

\subsection{An Adaptive Wiener Filtering Based Technique for an Automated Detection System}

Conventional noise reduction techniques usually do not work well for signals with short duration because they require statistical information on both the signal and the noise. AWF is used to reduce the noise in the data and enhance the defect signals. The de-noising process has two major steps. First step is removing unwanted low frequency noise components; using a 
simple zero-phase (HP) filter, with a cut-off frequency set (approximately) at the $3 \mathrm{~dB}$ bandwidth of the low-frequency noise component [18].

The second step is the removal of random noise which is not an easy task, because the spectrum of noise overlaps that of the defect signals. Lee Adaptive Weiner Filter (LAWF) is essentially an AWF that utilizes a known noise power and an estimate of the local variance of the signal at each data point. LAWF suffers from two major drawbacks; first, it requires prior knowledge of the noise power, which is rather undesirable, and second, its performance deteriorates when the signal-to-noise ratio (SNR) is low and the noise power is inaccurately known.

In order to overcome the above drawbacks of LAWF, an improved AWF that incorporates two modifications is introduced. First, the de-noising performance of the filter is improved by introducing a non-rectangular window to process weighted data samples, and second, a scheme for online estimation ${ }^{17}$ of noise power is incorporated.

Weighted adaptive wiener filtering (WAWF) is an improved version of LAWF which uses weighted data (with greater weight attached to the current data point than its neighbors), and more suitable windows like Gaussian, Blackman, Hamming, or Kaiser. The WAWF filtering shows better performance over the LAWF as shown in Fig 3.1 [18].

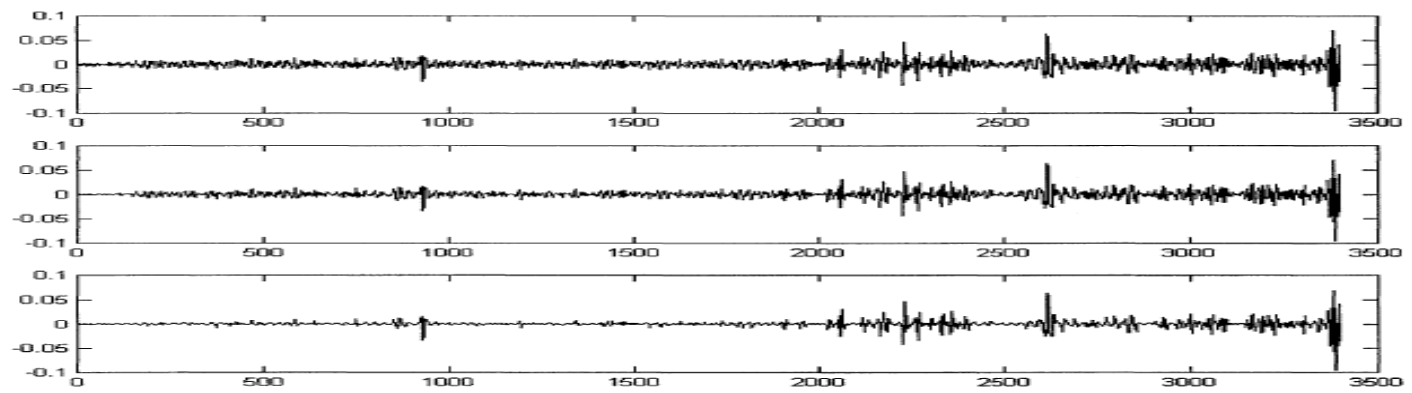

Figure 3. 1 (a): Original eddy current signal after high pass filtering (at the top), (b) Signal enhanced by LAWF(middle) (c) Signal enhanced by WAWF (end) [18].

${ }^{17}$ Online estimation of power is done using simple histogram to estimate $\sigma_{n}{ }^{2}$ 


\subsection{Proposed Improvements}

Although a number of automated analysis systems have been proposed over the years, their performance degrades due to high false alarm rates, poor detection and characterization. Removing sinusoidal interference using Cyclic MUISC algorithm and random noise using WAWF with help of dynamic thresholding to detect flaw signals can be a promising first step towards more reliable automated system.

Although the dynamic thresholding automated system significantly reduces the number of points that need to be further analyzed, low amplitude defect signals under the threshold $\lambda$ can be missed. Also, the preprocessing step considers only one source of low-frequency at $50 \mathrm{~Hz}$. Similarly, [18] considers only one source of low frequency noise with cut-off frequency at the $3 \mathrm{~dB}$ bandwidth of the low-frequency noise component.

The removal of unwanted low frequency noise components in both [16] and [18] require more attention since it assumes that there is only one source of low frequency noise and does not consider that this noise could be time varying in frequency and phase. Also, an additional preprocessing step is required to dissect the sources of distortion into deterministic (sinusoidal interference) and random noises using spectral analysis. The low-frequency sinusoidal interference parameters can be estimated and removed using well known DSP algorithms. 


\section{CHAPTER 4 SIGNAL PREPROCESSING}

\section{AND SINUSOIDAL PARAMETERS}

\section{ESTIMATION ALGORITHMS}

\subsection{Introduction}

The estimation of the parameters of a sinusoidal signal is an old problem and has been extensively studied in the literature. Many algorithms have been devised to estimate signal parameters for sinusoids in additive white noise. The success of those algorithms is typically demonstrated by means of simulated numerical examples.

One of the estimation methods used is the relaxed algorithm which is based on maximum likelihood estimation and nonlinear least square fitting [19]. The noise model considered in the Relaxed algorithm is zero mean white Gaussian noise. Using any different kind of noise model, the performance of the algorithm will degrade resulting in wrong estimations [20].

On the other hand, the proposed algorithm Cyclic-MUSIC has two main components. The first component acquires the initial estimation by using many known algorithms (MUSIC, linear regression with least squares estimates). The second component applies the initial estimates to a modified version of the relaxed algorithm and uses an FFT to obtain the improved estimates. 
The objective of signal preprocessing in this research is to extract important information from the data record and get the data ready for estimation. The original data record is passed through a series of preprocessing steps to get it ready for processing. Figure 4.1 shows a schematic diagram of the overall preprocessing module.

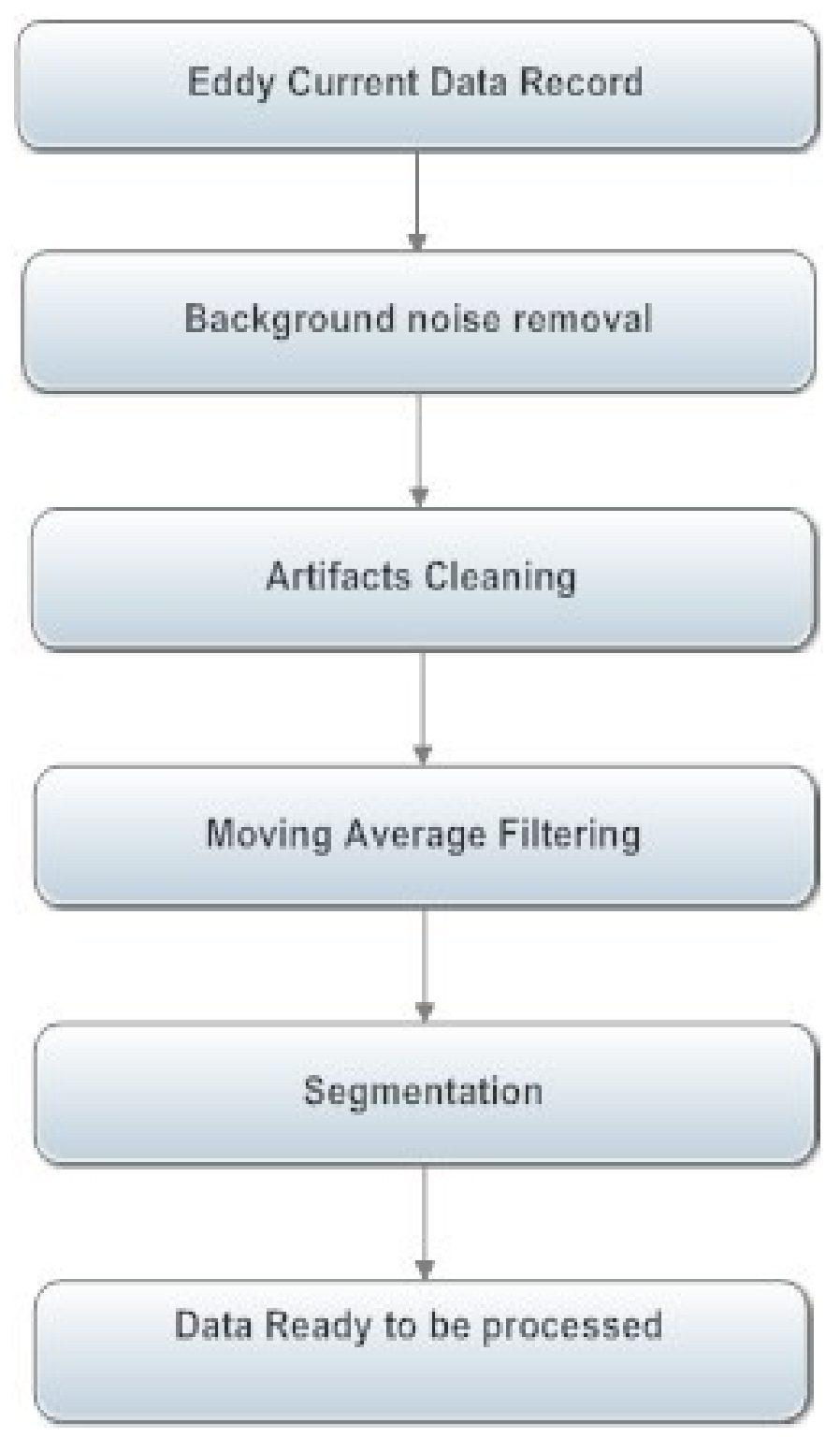

Figure 4. 1 Schematic Diagram of Signal Preprocessing. 


\subsection{Background Noise Removal}

The first step in the signal preprocessing process is to remove the background noise ${ }^{18}$ effect in the data record. The raw data has considerable variations in amplitude of a long duration compared to a typical duration of a flaw signal. The background amplitude variations are removed by using the strategy shown in Figure 4.2. Using a 256 moving non overlapping window at a time, a second order polynomial is fit to the data by least squares techniques. The fitted polynomial is then subtracted from the data record leaving a residual data stream free of background effect. A typical data record before and after background removal is shown in Figure 4.3.

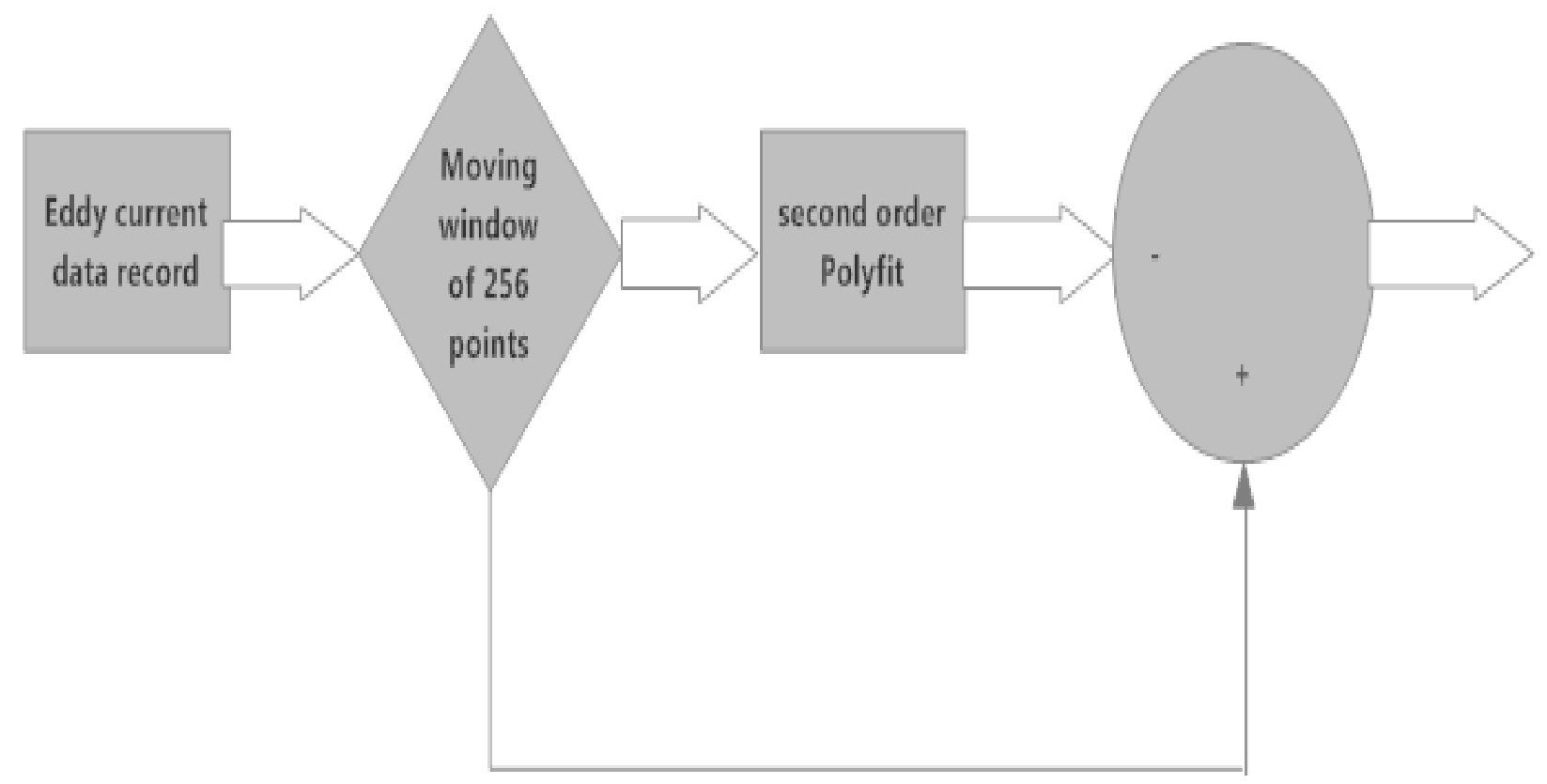

Figure 4. 2 Background Removal Block Diagram.

\footnotetext{
${ }^{18}$ Background noise is the trends and basis and the drift in the data record.
} 


\subsection{Artefacts Removal}

The objective of this step is to remove the $\operatorname{artefacts}^{19}$ from the data record. The artifacts exhibit high amplitude resulting from probe entering and exiting the tube. The removal process will reduce the range of data points that need to be analyzed and those that need to be discarded (for instance, data points at the beginning 'air to tube entry point' and end of the tube - 'tube exit point to air'). Since the support plate locations are known, the first support plate location is taken as a starting reference point of the data record and the location of the last support plate is taken as the ending reference point of the data record. Figure 4.3 and 4.4 show the original data record and the truncated version of the data record after the removal of the artefacts.
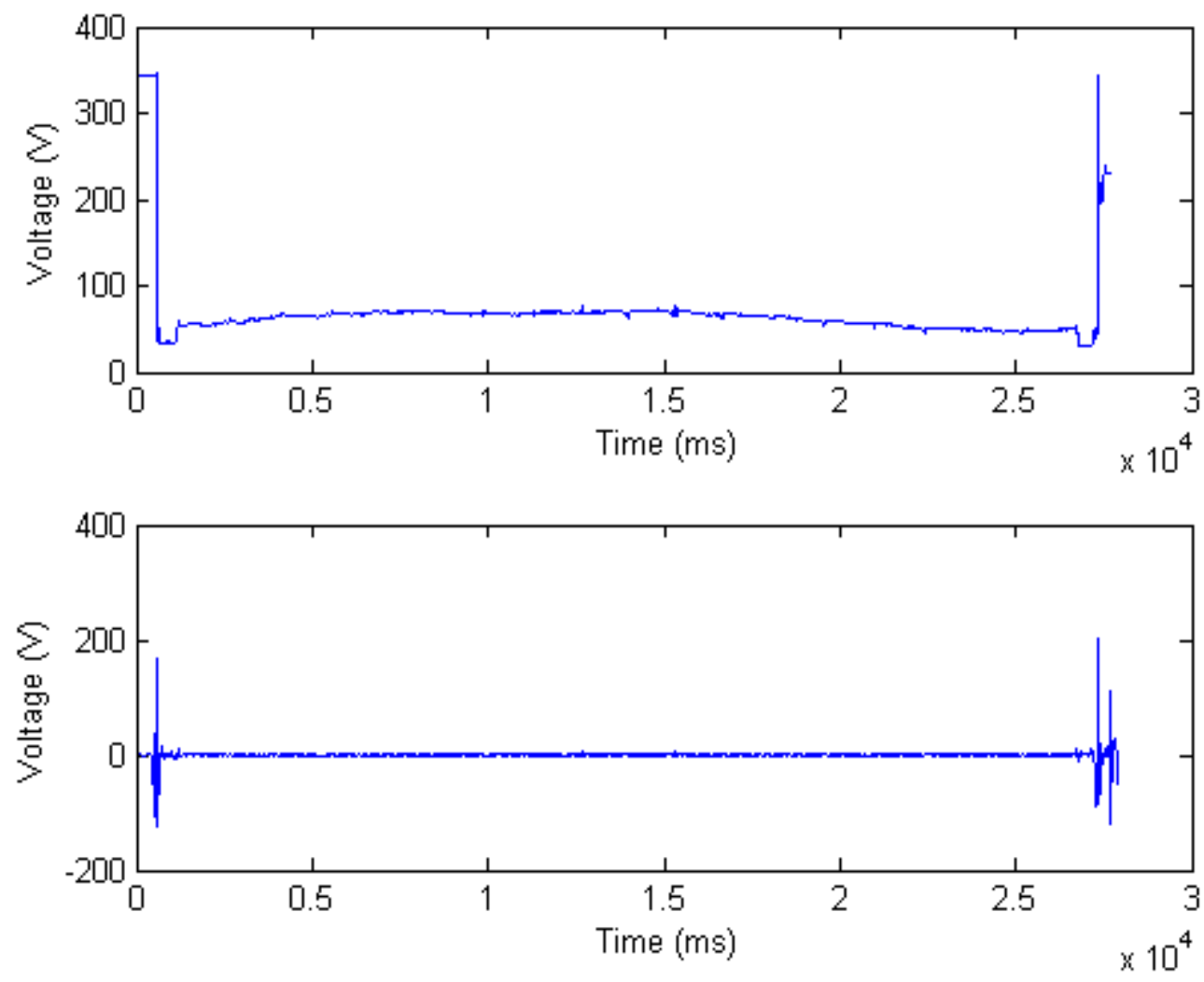

Figure 4. 3 Data Record Before and After Background Removal.

\footnotetext{
${ }^{19}$ Artefacts are the long amplitudes at the beginning and ending of data record generated by probe entering and exiting the tube.
} 


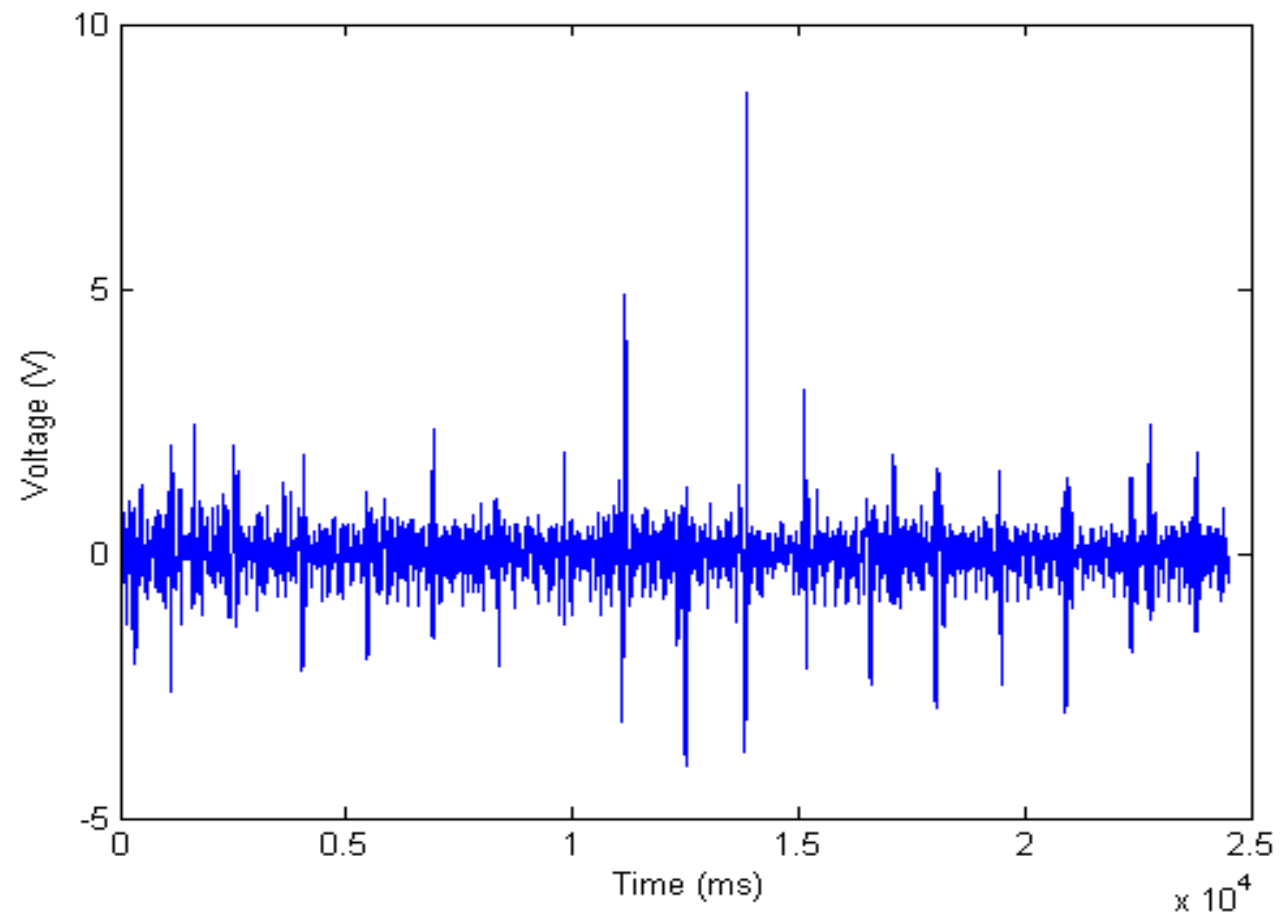

Figure 4. 4 Data Record after Artefacts Removal.

\subsection{Moving Average Filtering}

The moving average filter is used to remove the high-frequency ${ }^{20}$ noise riding on the potential defect signals in the vertical channel. Thus, this stage essentially acts as a low-pass filter.

The filter uses a 3-point window for smoothening purposes. This window length is short compared to the flaw signal duration to avoid any distortion to flaw signal. If $\mathrm{X}(\mathrm{k})$ represents a vertical channel data point in the original data, and $\mathrm{Y}(\mathrm{k})$ represents the corresponding data point in the smoothed signal, then we have equation (8):

$$
\mathrm{Y}(\mathrm{k})=[\mathrm{X}(\mathrm{k}-1)+\mathrm{X}(\mathrm{k})+\mathrm{X}(\mathrm{k}+1)] / 3
$$

\footnotetext{
${ }^{20}$ High frequency maybe caused by probe wobbling very fast or by the sampling equipment
} 
This moving average filter has a frequency response $1+\cos \omega$ where $\omega=\frac{2 \pi F}{F_{s}}$ and $F_{s}$ is the sampling frequency. The $3 \mathrm{~dB}$ cut-off is around $\frac{\pi}{3}$ which is equivalent to $\frac{F_{s}}{6}$. Figures 4.5 and 4.6 show the frequency response of the 3 point moving average and the effect of the moving average filter on one of the data segments.

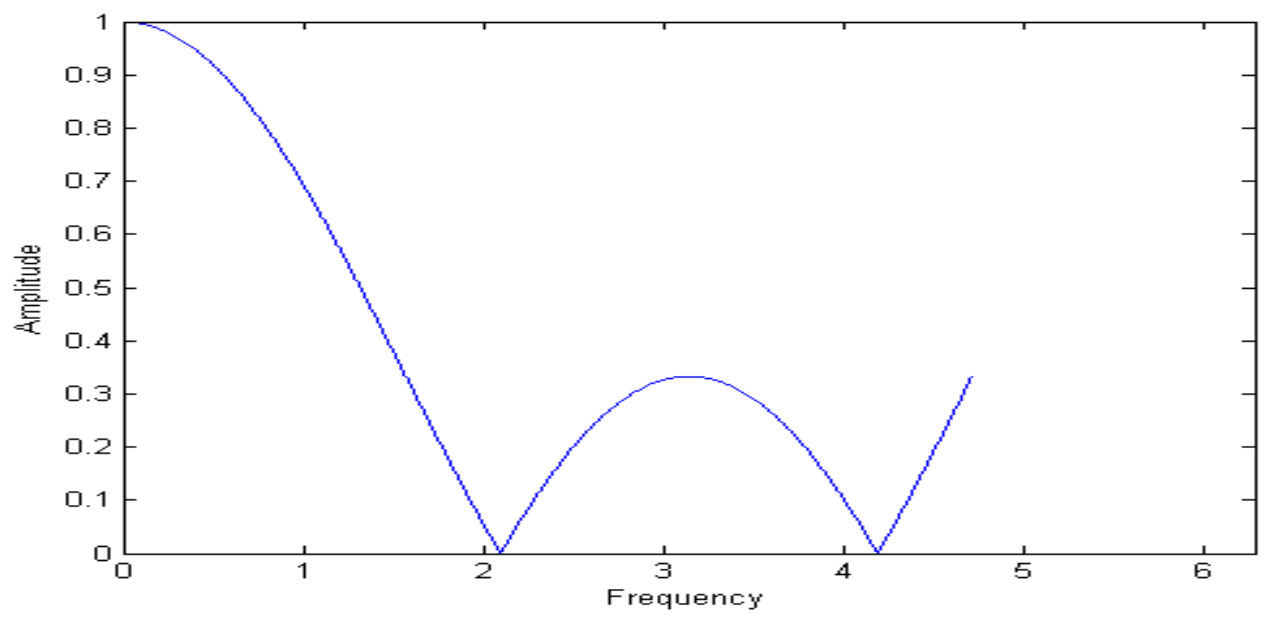

Figure 4. 5 Frequency Response of 3-Points Moving Average Filter.
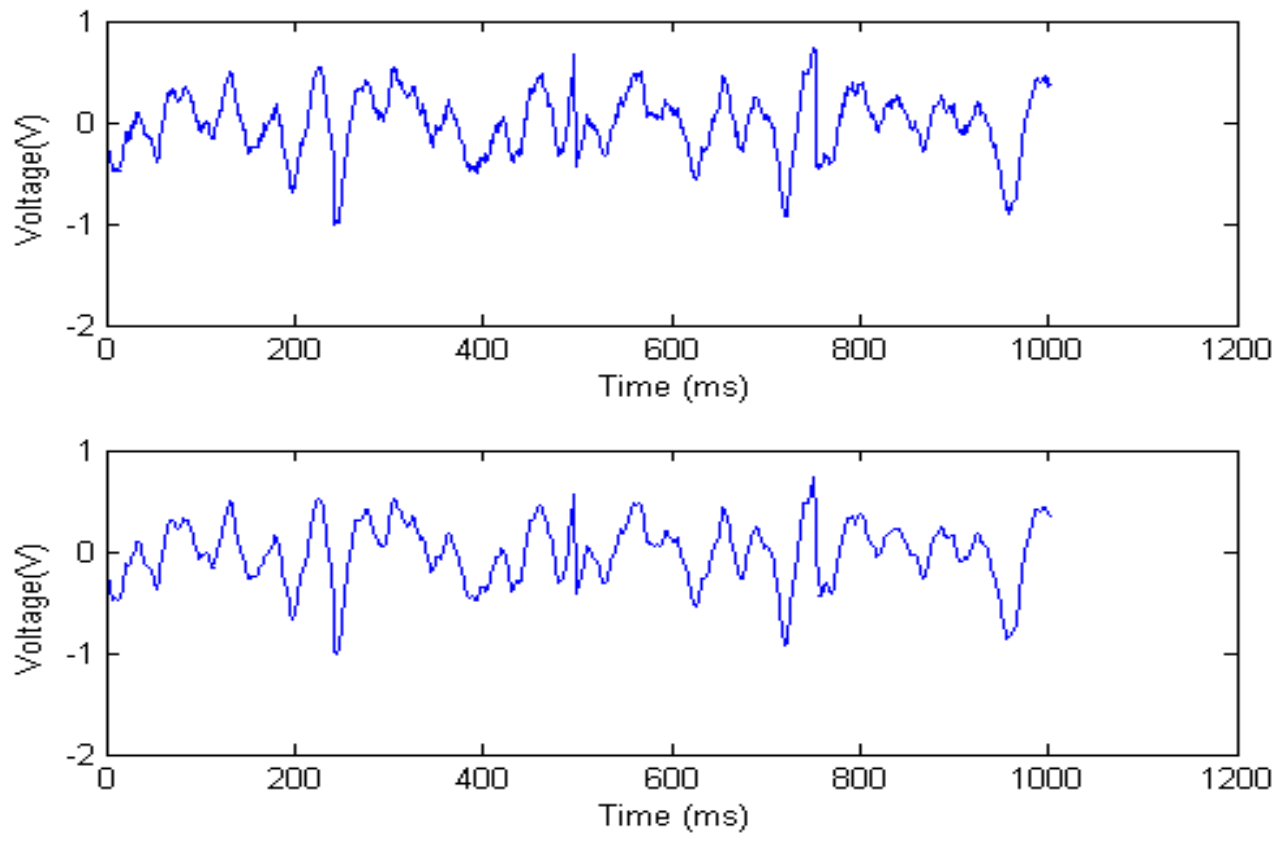

Figure 4. 6 Data Record Before and After Moving Average Filtering. 


\subsection{Segmentation}

The last step in the preprocessing stage is to segment the data record according to the number of support plates in the tube. The segmentation process eliminates the effect of the support plates' responses. The support plate locations are known and the duration of the support plate response is around 100 points. The distance between two support plates is about 1300 points, so we take each segment to be 1000 data points between two successive support plates, while preserving the support plate response. For each segment, we will estimate the sinusoidal noise parameters (frequency, amplitude and phase) and remove it from the data record. A typical data segment and its spectrum are shown in Figures 4.7 and 4.8. The amplitude spectrum shows that the two dominant frequencies are 21 and $8 \mathrm{~Hz}$.

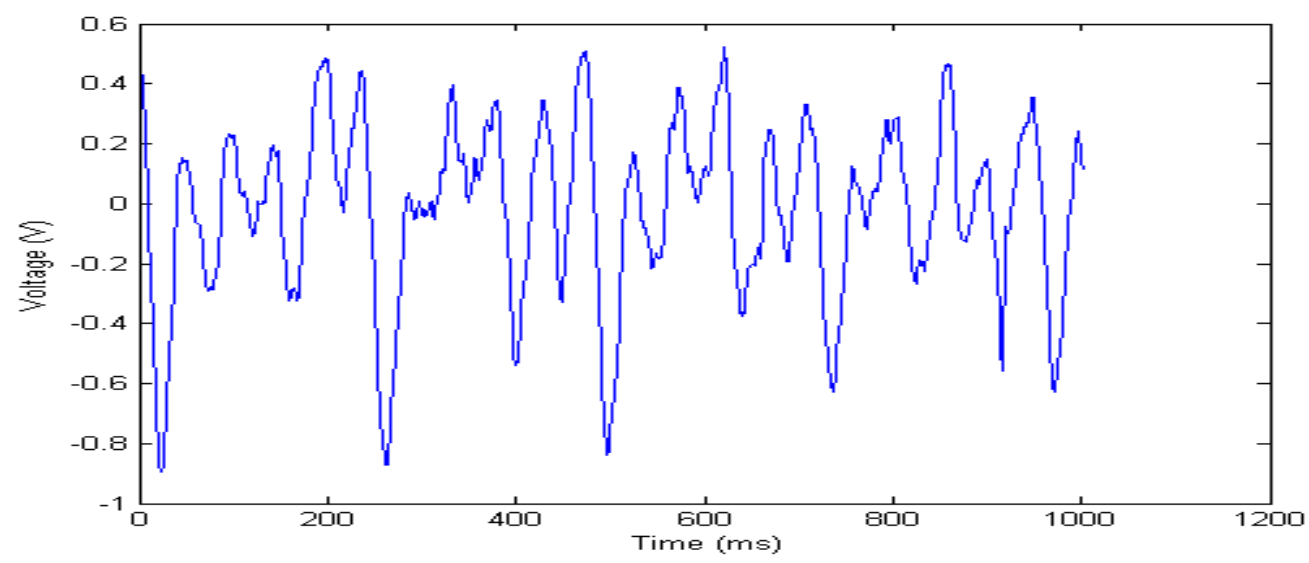

Figure 4. 7 Typical Segment Between Two Support Plates.

Figure 4.9 shows a detailed structure of a SG. The straight-legs of the tubes are supported by metal plates (H01-H07 and C01-C07) and the U-bend is supported by CU1, TOP, and HU1 [2]. As mentioned above, the data is segmented into 1000 points region between two support plates; those regions are indicated as 1-18 on Figure 4.9. Segments 1-7 make-up the cold-leg freespan; and segments 12 to 18 make-up the hot-leg freespan. The U-bend freespan consist of segments 
8-11, and for this study the tube-sheet ${ }^{21}$ (CTE to CTS and HTE to HTS) segments were not considered. Table 4.1 shows the assigned segment for each region.

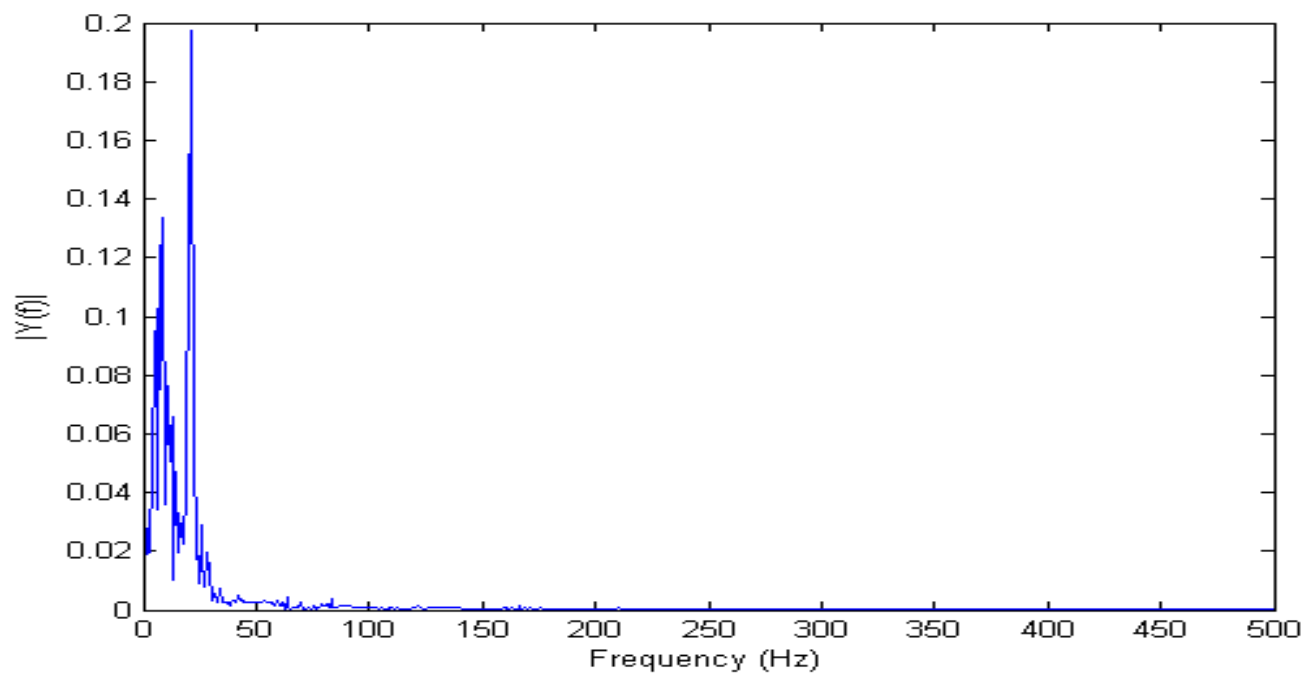

Figure 4. 8 Segment Amplitude Spectrum.

\begin{tabular}{|c|c|}
\hline Segment \# & Assigned Region \\
\hline Tube sheet & CTE to CTS \\
\hline 1 & CTS to C01 \\
\hline 2 & C01 to C02 \\
\hline 3 & C02 to C03 \\
\hline 4 & C03 to C04 \\
\hline 5 & C04 to C05 \\
\hline 6 & C05 to C06 \\
\hline 7 & C06 to C07 \\
\hline 8 & C07 to CU1 \\
\hline 9 & CU1 to TOP \\
\hline 10 & TOP to HU1 \\
\hline 11 & HU1 to H07 \\
\hline 12 & H07 to H06 \\
\hline 13 & H06 to H05 \\
\hline 14 & H05 to H04 \\
\hline 15 & H04 to H03 \\
\hline 16 & H03 to H02 \\
\hline 17 & H02 to H01 \\
\hline 18 & H01 to HTS \\
\hline Tube sheet & HTSto HTE \\
\hline
\end{tabular}

Table 4. 1 Assigned Segments for Each Region.

${ }^{21}$ A tube sheet is a plate, which is perforated with a pattern of holes designed to accept pipes or tubes. 


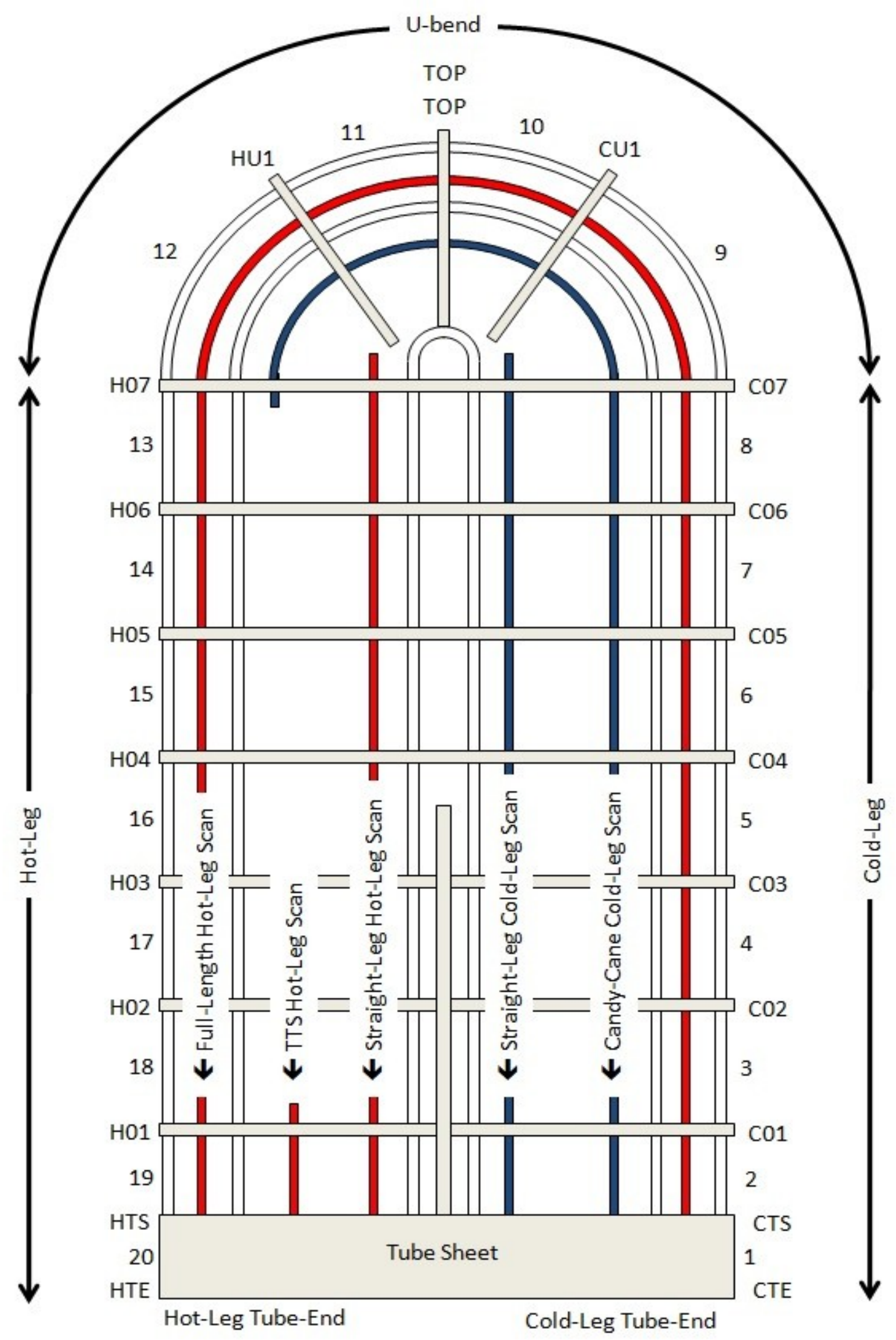

Figure 4. 9 Detailed Structure of a SG. Reproduced with permission Richard Lakhan [2]. 


\subsection{Problem Modeling}

Consider a signal consisting of a known number of complex sinusoids $\mathrm{K}$, so the sampled signal is expressed as [19]:

$$
y_{n}=\sum_{k=1}^{K} \alpha_{k} e^{j \theta_{k} n}+e_{n}
$$

where

$n=0,1 \ldots \ldots \ldots . . . N-1$, with $\mathrm{N}$ is the number of data samples

$\alpha_{k}=\left|\alpha_{k}\right| e^{j \phi_{k}}$ where $\left|\alpha_{k}\right|$ is the real valued amplitude and $\phi_{k}$ is the initial phase.

$\theta_{k}$ is the frequency parameter.

$e=\left[e_{0} e_{1} \ldots . . e_{N-1}\right]$

$y=\left[y_{0} y_{1} \ldots \ldots \ldots y_{N-1}\right]$,

$a\left(\theta_{k}\right)=\left[1 e^{j \theta_{k}} \ldots \ldots e^{j \theta_{k}(N-1)}\right]$

Then (1) can be expressed as:

$$
y=\sum_{k=1}^{K} \alpha_{k} a\left(\theta_{k}\right)+e
$$

where $\mathrm{y}$ is $1 \mathrm{xN}$ vector and $e$ is $1 \mathrm{xN}$ vector.

\subsection{Relaxed Algorithm}

Many notations are used in this algorithm, $(.)^{T}$ means the transpose, $(.)^{H}$ means conjugate transpose and $\|$.$\| means the Euclidean norm.$ 
Based on the assumption that noise is zero mean white Gaussian, The maximum likelihood estimator requires solving the following nonlinear least-squares fitting problem [19]:

$$
\{\hat{\alpha}, \hat{\theta}\}_{k=1}^{K}=\arg \min _{\left\{\alpha_{k}, \theta_{k}\right\}_{k=1}^{K}}\left|y-\sum_{k=1}^{K} \alpha_{k} e^{j \theta_{k} n}\right|^{2}
$$

The parameters $\left\{\alpha_{i}, \theta_{i}\right\}$ are estimated by minimizing the least squares criterion:

$$
\{\hat{\alpha}, \hat{\theta}\}_{k=1}^{K}=\arg \min _{\left\{\alpha_{k}, \theta_{k}\right\}_{k=1}^{K}}\left\|\hat{y}_{i}-\alpha_{i} a\left(\theta_{i}\right)\right\|^{2}
$$

where $\hat{y}_{i}=y-\sum_{k=1}^{K}{ }_{k \neq i} \hat{\alpha}_{k} a\left(\hat{\theta}_{k}\right)$

Minimizing the right side of (12) with respect to $\alpha_{i}$ yields:

$$
\hat{\alpha}_{i}=\frac{a^{H}\left(\theta_{i}\right) \hat{y}_{i}}{\left\|a\left(\theta_{i}\right)\right\|^{2}}
$$

Substituting the estimate of $\hat{\alpha}_{i}$ in (12) yields:

$$
\hat{\theta}_{i}=\arg \min _{\theta_{i}}\left\|P_{a\left(\theta_{i}\right)}^{\perp} \hat{y}_{i}\right\|^{2}=\arg \min _{\theta_{i}} \frac{a^{H}\left(\theta_{i}\right) \hat{y}_{i}}{\left\|a\left(\theta_{i}\right)\right\|^{2}}=\arg \max _{\theta_{i}} \frac{\left\|a^{H}\left(\theta_{i}\right) \hat{y}_{i}\right\|^{2}}{\left\|a\left(\theta_{i}\right)\right\|^{2}}
$$

$\hat{\alpha}_{i}$ is the magnitude and phase estimates

$\hat{\theta}_{i}$ is the frequency estimate

The algorithm uses maximum likelihood estimates in (13) and (14) and the estimated $\hat{y}_{i}[19,20]$ : 


$$
\hat{y}_{i}=y-\sum_{k=1}^{K}{ }_{k \neq i} \hat{\alpha}_{k} a\left(\hat{\theta}_{k}\right)
$$

The Relaxed algorithm postpones the estimation of the new parameters $\left\{\hat{\alpha}_{i}, \hat{\theta}_{i}\right\}$ until the initial estimated parameters $\left\{\hat{\alpha}_{k}, \hat{\theta}_{k}\right\}_{k=1}^{i-1}$ are good enough, and thus provides better initial estimates for the final cyclic optimization. The Relaxed algorithm is summarized in these steps:

- Step (1): Obtain $\left\{\hat{\alpha}_{1}, \hat{\theta}_{1}\right\}$ using a periodogram of y

- Step (2): Assume $\boldsymbol{K}=2$, compute $\hat{y}_{2}$ using equation (15) and $\left\{\hat{\alpha}_{1}, \hat{\theta}_{1}\right\}$ obtained in Step (1). Obtain $\left\{\hat{\alpha}_{2}, \hat{\theta}_{2}\right\}$ from $\hat{y}_{2}$ by using equation (13) and equation (14). Next, compute $\hat{y}_{1}$ using equation (15) and $\left\{\hat{\alpha}_{2}, \hat{\theta}_{2}\right\}$, and re-determine $\left\{\hat{\alpha}_{1}, \hat{\theta}_{1}\right\}$ from $\hat{y}_{1}$ as in equations (13) and (14) above. Iterate the previous two sub steps until convergence is achieved.

- $\quad$ Step (3): Assume $K=3$. Compute $\hat{y}_{3}$ using equation (15) and $\left\{\hat{\alpha}_{i}, \hat{\theta}_{i}\right\}_{i=1}^{2}$ obtained in Step (2). Obtain $\left\{\hat{\alpha}_{3}, \hat{\theta}_{3}\right\} 3$ from $\hat{y}_{3}$. Next, compute $\hat{y}_{1}$ using equation (15) and $\left\{\hat{\alpha}_{i}, \hat{\theta}_{i}\right\}_{i=2}^{3}$, and re-determine $\left\{\hat{\alpha}_{1}, \hat{\theta}_{1}\right\}$ from $\hat{y}_{1}$. Then, compute $\hat{y}_{2}$ using equation (15) and $\left\{\hat{\alpha}_{i}, \hat{\theta}_{i}\right\}_{i=1,3}$ and re-determine $\left\{\hat{\alpha}_{2}, \hat{\theta}_{2}\right\}$ from $\hat{y}_{2}$. Iterate the previous three sub steps until convergence.

The practical convergence can be determined by comparing the difference between two consecutive iterations. If the difference $\mathrm{t}$ is less than or equal to 0.001 , terminate the iterations.

- Remaining Steps: Continue similarly until $K$ is equal to the desired number of sinusoids $[20,21$, and 22]. 
The pseudo-code for Relaxed algorithm is shown below:

$* * * * * * * * * * * * * * * * * * * * * * * * * * * * * * * * * * * * * * * * * * * * * * *$

initialize

$\hat{\alpha}_{k}=0, k=1,2, \ldots, K$

$\left(\hat{\theta}_{k}\right.$ values are not required)

for $i=1,2 \ldots K$

$\hat{y}_{i}=y-\sum_{k=1}^{K} \hat{\alpha}_{k} a\left(\hat{\theta}_{k}\right)$

$\hat{\theta}_{i}=\arg \max _{\theta} \frac{\left\|a^{H}(\theta) \hat{y}_{i}\right\|^{2}}{\|a(\theta)\|^{2}}$

$\hat{\alpha}_{i}=\frac{a^{H}\left(\hat{\theta}_{i}\right) \hat{y}_{i}}{\left\|a\left(\hat{\theta}_{i}\right)\right\|^{2}}$

repeat

for $j=1,2 \ldots i$

$\hat{y}_{j}=y-\sum_{m=1}^{i} \hat{\alpha}_{m} a\left(\hat{\theta}_{m}\right)$

$\hat{\theta}_{j}=\arg \max _{\theta} \frac{\left\|a^{H}(\theta) \hat{y}_{j}\right\|^{2}}{\|a(\theta)\|^{2}}$

$\hat{\alpha}_{j}=\frac{a^{H}\left(\hat{\theta}_{j}\right) \hat{y}_{j}}{\left\|a\left(\hat{\theta}_{j}\right)\right\|^{2}}$

end for

until convergence

end for

Flowchart for the Relaxed Algorithm is shown in Figure 4.10. 


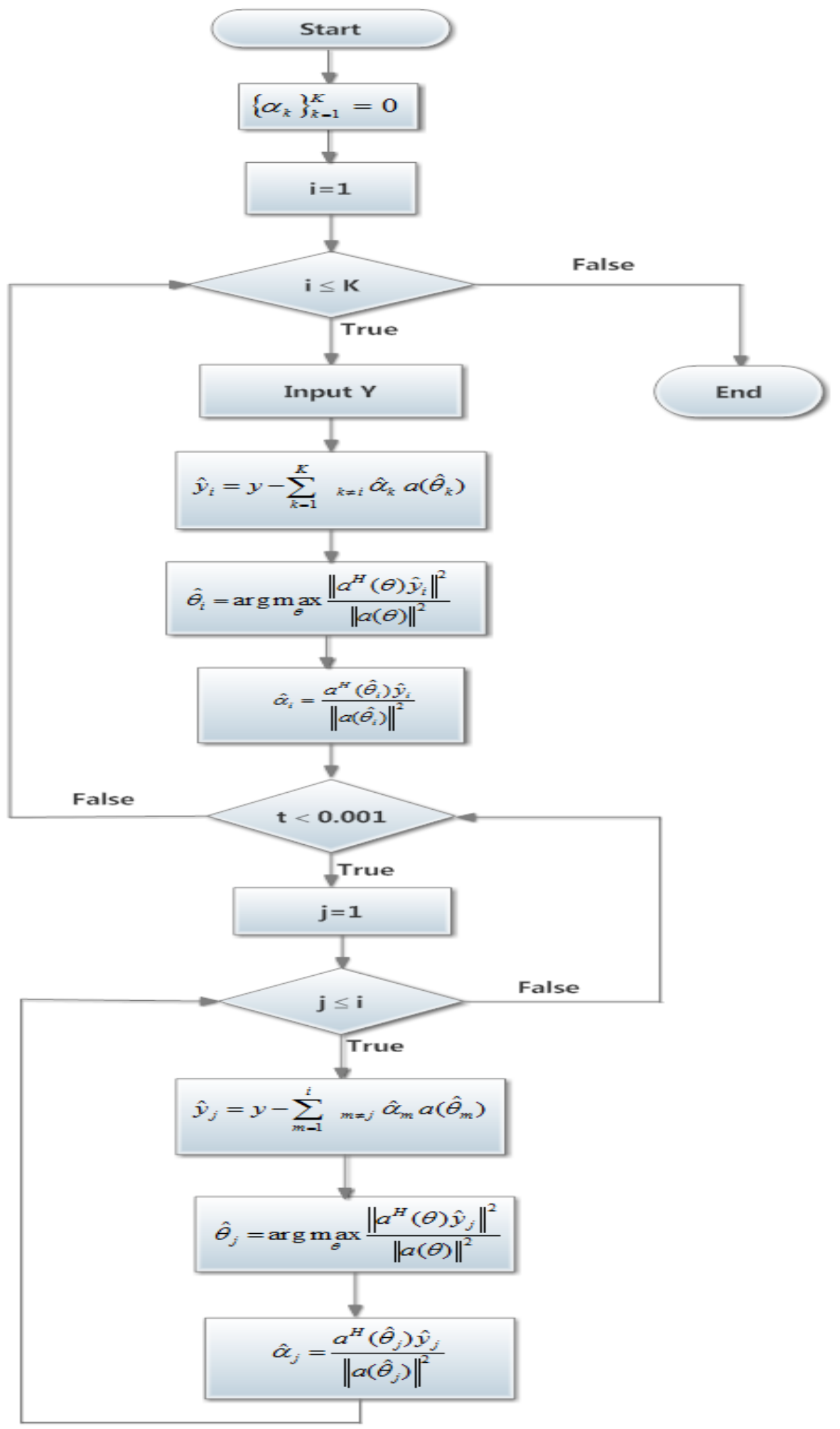

Figure 4.10 Flowchart for Relaxed Algorithm. 


\subsection{Initial Estimates and Cyclic-MUSIC Algorithm}

Although, the Relaxed algorithm relies on the maximum likelihood estimator in estimating the sinusoidal parameters, Cyclic-MUSIC tackles the problem in a slightly different manner using MUSIC and a modified version of Relaxed algorithms.

The cyclic-Music algorithm has two main components. The first component determines

the initial estimates (frequency, magnitude and phase) $\left\{\hat{\theta}_{i}, \hat{\alpha}_{i}\right\}_{i=1}^{K}$ for each sinusoidal signal in the data by using Multiple Signal Classification (MUSIC) algorithm, linear regression and least square estimates. The second component uses the initial estimates and applies it to a modified version of the Relaxed Algorithm with Fast Fourier Transform (FFT) to obtain improved estimates.

\subsubsection{Initial Estimates Computation}

The initial estimates determination process consists of three main steps:

1. Auto correlation matrix

2. Frequency estimation

3. Magnitude and phase estimation

\subsubsection{Auto-Correlation Matrix}

The first step in this process is determining the correlation matrix for the data. The correlation matrix $\mathrm{X}_{\text {mod }}$ is based on a modified covariance rectangular Toeplitz matrix. It generates an autocorrelation estimate for the data of length $\mathrm{N}$, which uses the forward and backward prediction error estimates based on an $m^{\text {th }}$ order prediction error model. The forward backward prediction model is explained in Appendix A. 
$\mathrm{X}_{\text {mod }}$ is $2(\mathrm{~N}-\mathrm{m})$ by $(\mathrm{N}+1)$ matrix, where $\mathrm{n}$ is the length of the data and the default value for $\mathrm{m}$ is N/4. A sample correlation matrix (16) is shown below [24].

$X_{\mathrm{mod}}=\left[\begin{array}{ccc}x(N+1) & \ldots & x(1) \\ \vdots & \ddots & \vdots \\ x(N-m) & \ldots & x(m+1) \\ \vdots & \ddots & \vdots \\ x(N) & \ldots & x(N-m) \\ x *(1) & \ldots & x *(m+1) \\ \vdots & \ddots & \vdots \\ x *(m+1) & \ldots & x *(N-m) \\ \vdots & \ddots & \vdots \\ x *(N-m) & \ldots & x *(N)\end{array}\right]$

\subsubsection{Frequency Estimation}

The multiple signal classification (MUSIC) spectral estimation algorithm is used to determine the frequencies of the sinusoids. The MUSIC algorithm performs eigendecomposition on the correlation matrix $X_{\text {mod }}$ to estimate the frequencies of sinusoids observed in additive white noise. The MUSIC algorithm is described in Appendix A.

The MUSIC spectrum estimate is defined in equation (17) as [24, 25]:

$$
P_{X X}^{M U S I C}(f)=\frac{1}{\sum_{k=p+1}^{N}\left|S^{H}(f) v_{k}\right|^{2}}=\frac{1}{s^{H}(f) V(f) V^{H}(f) s(f)}
$$


where $s(f)$ is the complex sinusoidal vector, and $V$ is the matrix of the noise subspace. $P_{X X}^{M U S I C}$ is sharply peaked at the frequencies of the sinusoidal components of the signal, and hence the frequency of its peaks are taken as the MUSIC frequency estimates.

\subsubsection{Determination of the Magnitude and Phase}

Using the estimated frequency for each sinusoid in the data, the magnitude and phase are estimated using linear regression and least square estimates.

Form a design matrix A equation (18) with first column is the DC term in the data (either one or mean value). For each sinusoid, we need two columns (one sine and one cosine), matrix A is shown below:

$$
A=\left[\begin{array}{cccc}
1 & \cos \left(2 \pi f_{1}\right) & \sin \left(2 \pi f_{1}\right) & \ldots \\
\vdots & \vdots & \vdots & \vdots \\
\vdots & \vdots & \vdots & \vdots \\
1 & \cos \left(2 \pi f_{1} n\right) & \sin \left(2 \pi f_{1} n\right) & \ldots
\end{array}\right]
$$

Find the least square estimates using the $\backslash$ matrix operation, we have:

$$
\chi=A \backslash y
$$

where $\mathrm{y}$ is the data vector.

$$
\chi=\left[\begin{array}{c}
x(1) \\
x(2) \\
x(3) \\
\vdots \\
\vdots
\end{array}\right]
$$

The magnitude and phase are determined in the following two steps: 
Amplitude vector $V_{\text {mag }}=\left[\begin{array}{c}\sqrt{x(2)^{2}+x(3)^{2}} \\ \sqrt{x(4)^{2}+x(5)^{2}} \\ \vdots \\ \vdots\end{array}\right]$

Phase Vector $P_{\text {phase }}=\left[\begin{array}{c}a \tan (-x(3), x(2)) \\ a \tan (-x(5), x(4)) \\ \vdots \\ \vdots\end{array}\right]$

$V_{\text {mag }}$ and $P_{\text {phase }}$ equations (21) and (22) are the estimated magnitudes and phases for the each sinusoid in the data. A flowchart of the initial conditions estimation process is shown Figure 4.11 .

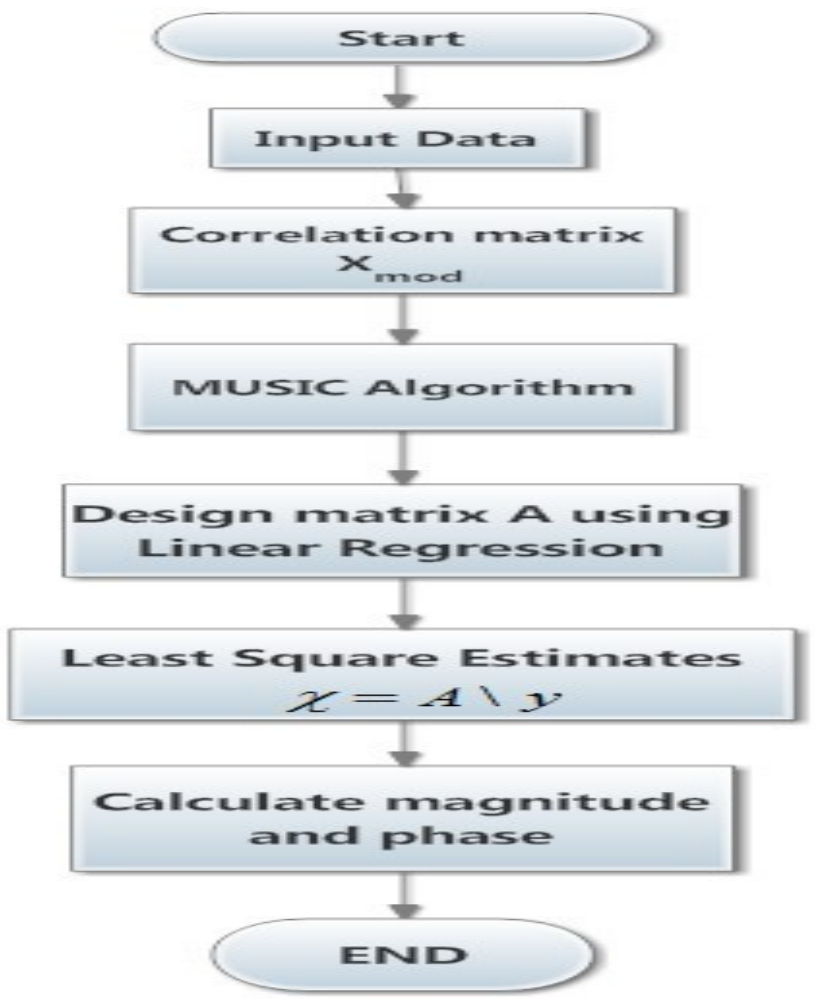

Figure 4. 11 Flowchart of the Initial Conditions Estimation Process. 


\subsubsection{Cyclic-MUSIC Algorithm}

The Cyclic-Music algorithm relies on the initial estimates obtained using the flowchart of Figure 4.11. These estimates are inputted to a modified version of the Relaxed algorithm. The modification of the Relaxed algorithm incorporates the FFT which leads to improved frequency, amplitude and phase estimates. A pseudo-code for the Cyclic-MUSIC algorithm is shown below and Figure 4.12 shows the flowchart of the Cyclic-MUSIC algorithm.

The pseudo-code for the Cyclic-MUSIC:

- Initial estimates

$K=1,2, \ldots K$ number of $\sin$ usoids

$\hat{\theta}_{k}$ initial estimated frequency

$\hat{\alpha}_{k}=\left|\hat{\alpha}_{k}\right| e^{j \phi}$ initial estimated magnitude and phase

for $k=1,2 \ldots \ldots K$

$\hat{y}_{k}=y-\sum_{k=1}^{K} \hat{\alpha}_{k} a\left(\hat{\theta}_{k}\right)$

$f f t\left(\hat{y}_{k}\right)$

get the max peak

extract magnitude and phase

end for 


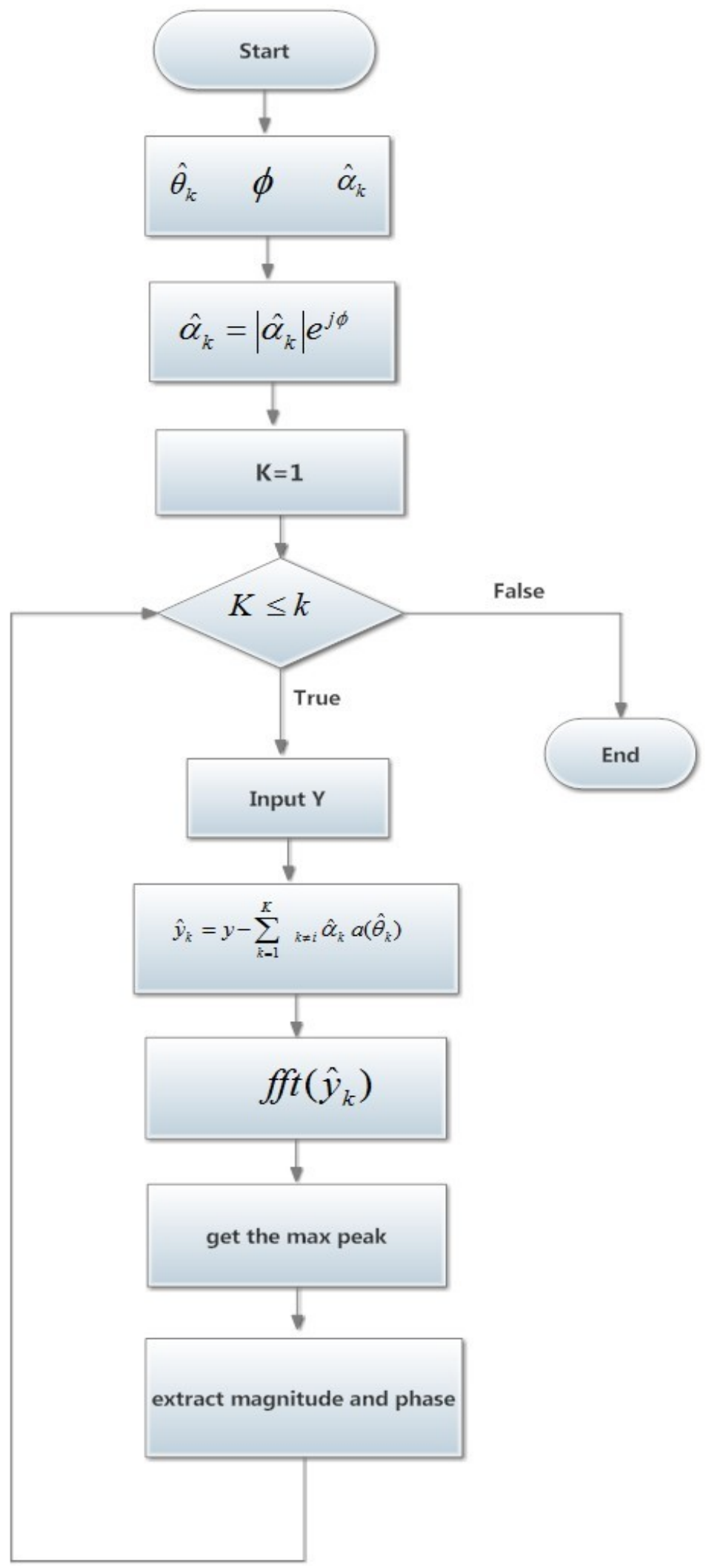

Figure 4. 12 Flowchart of Cyclic-MUSIC Algorithm. 


\subsection{CRLB for the MUSIC Algorithm}

The Cramer-Rao Lower Bound (CRLB) sets a lower bound on the variance of any unbiased estimator. It is useful as follows [30]:

1. If we find an estimator that achieves the CRLB, then we know that we have found a Minimum Variance Unbiased Estimator (MVUE).

2. An estimator that (asymptotically) achieves the CRLB is (asymptotically) efficient. The purpose of this section is to show that the MUSIC, root-MUSIC and linear least squares achieve CRLB. In addition, this section uses the local notation in the equations shown below.

The MUSIC algorithm performs an eigen-decomposition on the correlation matrix to estimate the frequencies of sinusoids. The first step in acquiring the CRLB for the music algorithm is to determine the CRLB for the deterministic model. The CRLB for deterministic model [26, 27, and 28]:

$C R L B_{\mathrm{det}}(\varphi)=\frac{\sigma^{2}}{2 N}\left[\operatorname{Re}\left\{\left(D^{H}(\varphi) P_{A}^{\perp}(\varphi) D(\varphi)\right) \circ \hat{R}^{T}{ }_{S S}\right)\right]^{-1}$

Where $\varphi$ is the unbiased estimator of the bounded frequency.

$a\left(\varphi_{k}\right)=\left[1 e^{j \varphi_{k}} \ldots \ldots e^{j \varphi_{k}(N-1)}\right]$

$A(\varphi)=\left[a\left(\varphi_{1}\right), a\left(\varphi_{2}\right), \ldots \ldots, a\left(\varphi_{k}\right)\right]$

$N$ is the length of the data

The projection on the null-space of $\mathrm{A}(\varphi)$ :

$P_{A}^{\perp}=I-A(\varphi) A^{+}(\varphi)$ 
The signal sample correlation matrix:

$\hat{R}_{S S}=\frac{1}{N} \sum_{n=1}^{N} s(n) s^{H}(n)=\frac{1}{N} S S^{H}$

And the matrix of partial derivatives with respect to $\varphi$ :

$D(\varphi)=\left[\frac{\partial a(\varphi)}{\partial \varphi_{1}}, \frac{\partial a(\varphi)}{\partial \varphi_{2}}, \frac{\partial a(\varphi)}{\partial \varphi_{3}}, \ldots \ldots, \frac{\partial a(\varphi)}{\partial \varphi_{n}}\right]$

\subsubsection{Asymptotic Covariance Matrix for Root-MUSIC}

Assuming $\mathrm{N}$ is large, the asymptotic covariance matrix for the Root- MUSIC algorithm is [27, 28, and 29]:

$C_{\text {Root-music }}=\frac{\sigma^{2}}{2 N}\left(\Re_{A}(\varphi) \circ I\right)^{-1} \cdot \operatorname{Re}\left\{\Re_{A}(\varphi) \circ\left(R_{S S}{ }^{-1}+\sigma^{2} R_{S S}^{-1}\left(A^{H}(\varphi) A(\varphi)\right)^{-1} R_{S S}^{-1}\right)^{T}\right\}\left(\Re_{A}(\varphi) \circ I\right)^{-1}$

Where $\mathfrak{R}_{A}=D^{H}(\varphi) P_{A}^{T}(\varphi) D(\varphi)$

The covariance matrix of the Root-MUSIC estimates $C_{\text {Root-music }}$ is bounded from below by $C R L B_{\text {det }}(\varphi)$. If the sinusoidal signals in the noisy data are not correlated, Rss is diagonal and for $N \rightarrow \infty$ we get: $\left.\left.\left(C_{\text {music }}(\varphi)\right)\right)_{i, i}=\left(C R L B_{\text {det }}(\varphi)\right)\right)_{i, i}$

\subsubsection{CRLB for Linear Least Squares Estimates}

The data model can be written in matrix notation as [30]:

$X=H \theta+w$ 
where $\mathrm{w}$ is the additive white Gaussian noise and $\mathrm{H}$ is a full rank matrix with orthogonal rows and columns.

So $p(x ; \theta)=\frac{1}{\left(2 \pi \sigma^{2}\right)^{N / 2}} \exp \frac{(X-H \theta)^{T}(X-H \theta)}{2 \sigma^{2}}$

Using the CRLB equation to obtain the MVU (minimum variance unbiased) for $\theta$, the MVU estimator, $\hat{\theta}=g(x)$ will satisfy:

$\frac{\partial \ln p(x ; \theta)}{\partial \theta}=I(\theta)(g(x)-\theta)$

Where $\mathrm{I}(\theta)$ is the Fisher information matrix whose elements are

$[I]_{i j}=-E\left[\frac{\partial^{2} \ln p(x ; \theta)}{\partial \theta_{i} \partial \theta_{j}}\right]=\frac{1}{\sigma^{2}} H^{T} H$

Taking the first derivative with respect to $\theta$

$$
\frac{\partial \ln p(x ; \theta)}{\partial \theta}=\underbrace{\frac{1}{\sigma^{2}} H^{T} H}_{I(\theta)}[\underbrace{\left(H^{T} H\right)^{-1} H^{T} X}_{g(X)}-\theta]
$$

So we have $\hat{\theta}=g(X)=\left(H^{T} H\right)^{-1} H^{T} X$

And the covariance matrix of $\hat{\theta}$ now becomes:

$$
C_{\hat{\theta}}=I^{-1}(\theta)=\sigma^{2}\left(H^{T} H\right)^{-1}
$$

The frequency estimation using MUSIC algorithm achieves the CRLB asymptotically and the estimation of magnitude and phase achieves CRLB and they are the MVUE. 


\section{CHAPTER 5 IMPLEMENTATION AND}

\section{RESULTS}

\subsection{Cyclic-MUSIC Algorithm Implementation}

The algorithms used in this research were implemented in MATLAB. The analysis is a multistep process which consists of preprocessing, noise estimation and noise removal steps.

The original data record must be first preprocessed before applying the cyclic-MUSIC algorithm. The preprocessing steps, as discussed in 4.2 to 4.5 , extract the meaningful information and reduce the number of data points to be analyzed. The first preprocessing step is to curve fit the data with 256 points moving window and subtract it from the original data to remove the noise in the background and to compensate for the drift in the data record. The second preprocessing step is to remove the artefacts from the data record caused by the probe entering and exiting the tube. The third step is applying a moving average filter to the data record in order to remove the high frequency riding on top of the data points. Finally, segmenting the data record according to the support plates' locations, so every 1000 point segment is between two consecutive support plates. The preprocessing strategy is outlined in the flowchart shown in Figure 4.1.

The first stage in the cyclic-MUSIC algorithm determines the initial estimates of the sinusoidal parameters (frequency, magnitude and phase). This process initially determines the 
modified covariance matrix equation (16) from the data record and applies it to the MUSIC algorithm to estimate the frequencies. Linear regression and least square estimates are used to compute the magnitude and phase. Finally, the initial estimates are applied to the CyclicMUSIC algorithm to obtain improved estimated parameters. The initial estimates computation process and Cyclic-MUSIC algorithm are outlined in flowcharts in Figures 4.11 and 4.12.

The removal process generates the sinusoidal waveforms using the estimated parameters from the Cyclic-Music algorithm and subtracts them from the original data segments.

The SG data analysis revealed that we might have multiple sources that are generating low frequency sinusoidal noise; we have to consider estimating more than one sinusoid. We are considering estimating:

- One sinusoid

- Two sinusoids

- Four sinusoids

Three methods for removing the low-frequency sinusoidal noise have been examined in this research:

- Using all the estimated parameters for each segment.

- Using the tube average frequency for each segment

- Using the tube average frequency and average magnitude for the whole tube data record

The performance assessment of the cyclic-Music algorithm is based on power and correlation:

1- Power analysis which measures the reduction of power after the removal of the estimated sinusoidal interference for one, two and four sinusoids. 
2- Correlation analysis which measures the change in the flaw signal after the removal of one, two and four sinusoids.

\subsection{Estimation Analysis}

The analysis was done on the data provided for us by AECL; the analysis of the results for TUBE 1 and TUBE 2 are presented. Estimation Tables for TUBE 3 to TUBE 7 are listed in Appendix C.

The results obtained by estimating one, two and four sinusoids for TUBE 1 and TUBE 2 are shown in tables 5.1 to 5.6. While investigating the estimation of one sinusoid for TUBE 1 and TUBE 2, we notice two repeated frequencies $21 \mathrm{~Hz}$ and $8 \mathrm{~Hz}$ in TUBE 1 and $19 \mathrm{~Hz}$ and $25 \mathrm{~Hz}$ in TUBE 2. Similarly, two and four sinusoids estimation show that the segments have two common frequencies except segment 6,17 and 18 in TUBE 1 . Also, segment 5 in TUBE 2 has different frequencies. These observations support the fact that the TUBE contains more than one low-frequency sinusoidal interference and they could be generated from two different sources.

Figures 5.1 and 5.2 show the bar plot for the frequency and magnitude for the one sinusoid estimation (TUBE1). The fluctuation of frequency and magnitude as shown in Figure5.1 and 5.2 can be caused by many factors such as geometric variations from segment to segment inside the tube, mechanical noise in some sections of the tube, probe wobble and interference from support plates.

In addition, after investigating the estimation of four sinusoids, we notice that estimating four sinusoids from the data is basically estimating the whole data as sum of sinusoids; this reduces the power and removes all the features of the flaw signals. More attention is paid to one and two sinusoids. 


\section{Frequency F1(Hz)}

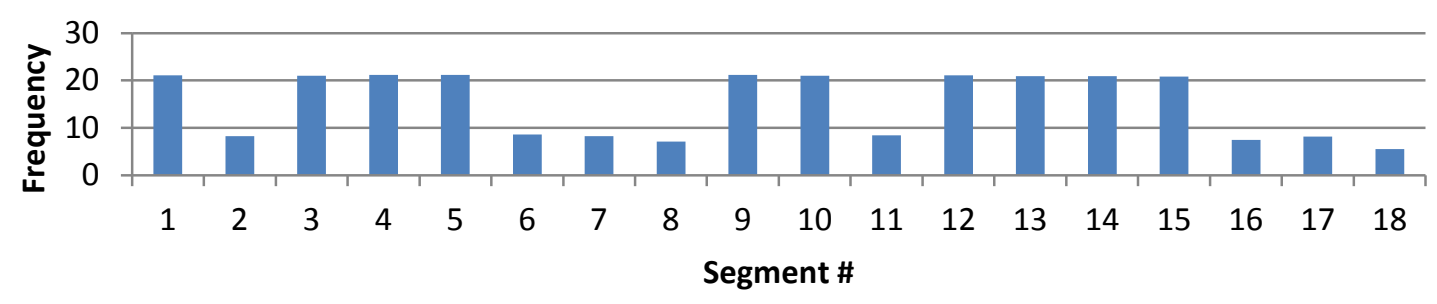

Figure 5. 1 Bar Plot for Frequency F1 (TUBE 1).

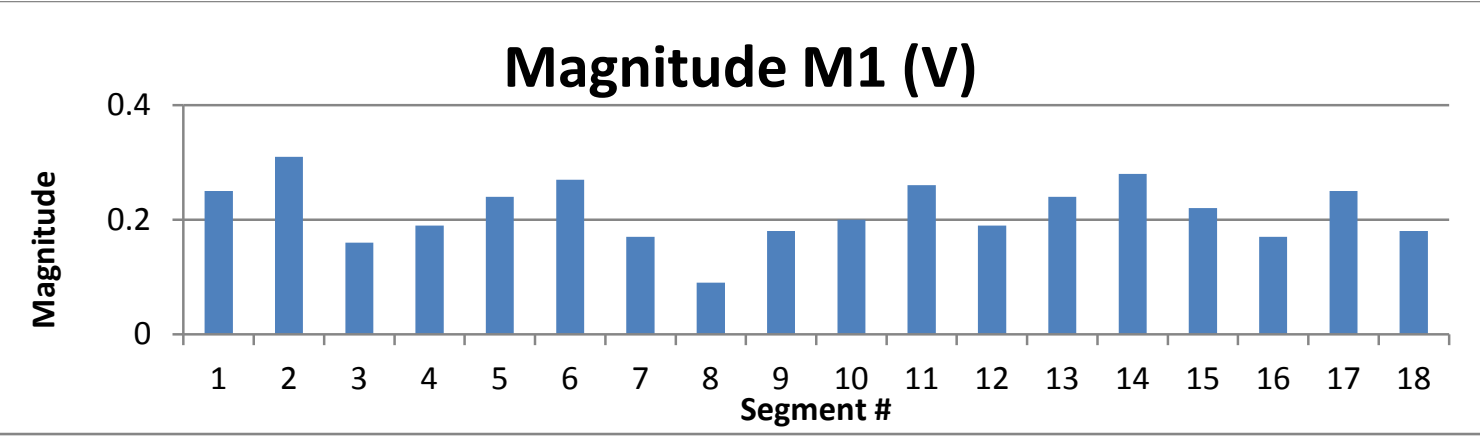

Figure 5. 2 Bar Plot for Magnitude M1 (TUBE 1).

\begin{tabular}{|c|c|c|c|}
\hline Segment \# & $\begin{array}{c}\text { Frequency } \\
\text { F1(Hz) }\end{array}$ & $\begin{array}{c}\text { Magnitude } \\
\text { M1(V) }\end{array}$ & $\begin{array}{c}\text { Phase } \\
\text { Ph1(Radians) }\end{array}$ \\
\hline 1 & 21.1 & 0.25 & 2.91 \\
\hline 2 & 8.3 & 0.31 & 2.44 \\
\hline 3 & 21 & 0.16 & -1.48 \\
\hline 4 & 21.2 & 0.19 & 2.21 \\
\hline 5 & 21.2 & 0.24 & 0.54 \\
\hline 6 & 8.6 & 0.27 & 3.08 \\
\hline 7 & 8.3 & 0.17 & 2.43 \\
\hline 8 & 7.1 & 0.09 & -1.61 \\
\hline 9 & 21.2 & 0.18 & -2.75 \\
\hline 10 & 21 & 0.20 & -1.04 \\
\hline 11 & 8.4 & 0.26 & -2.39 \\
\hline 12 & 21.1 & 0.19 & 3.08 \\
\hline 13 & 20.9 & 0.24 & 1.54 \\
\hline 14 & 20.9 & 0.28 & -0.38 \\
\hline 15 & 20.8 & 0.22 & -3.11 \\
\hline 16 & 7.5 & 0.17 & -2.08 \\
\hline 17 & 8.2 & 0.25 & 2.52 \\
\hline 18 & 5.5 & 0.18 & -2.15 \\
\hline
\end{tabular}

Table 5. 1 Frequencies, Magnitudes and Phases for One Sinusoid Estimation (TUBE 1). 


\begin{tabular}{|c|c|c|c|c|c|c|}
\hline $\begin{array}{c}\text { Segment } \\
\#\end{array}$ & $\begin{array}{c}\text { Frequency } \\
\text { F1(Hz) }\end{array}$ & $\begin{array}{c}\text { Frequency } \\
\text { F2(Hz) }\end{array}$ & $\begin{array}{c}\text { Magnitude } \\
\text { M1(V) }\end{array}$ & $\begin{array}{c}\text { Magnitude } \\
\text { M2(V) }\end{array}$ & $\begin{array}{c}\text { Phase } \\
\text { Ph1(Radians) }\end{array}$ & $\begin{array}{c}\text { Phase } \\
\text { Ph2(Radians) }\end{array}$ \\
\hline 1 & 21.1 & 7.8 & 0.25 & 0.11 & 2.94 & -0.86 \\
\hline 2 & 21.2 & 8.2 & 0.21 & 0.31 & 0.24 & 2.41 \\
\hline 3 & 21.1 & 8.3 & 0.16 & 0.14 & -1.35 & 2.15 \\
\hline 4 & 21.2 & 8.3 & 0.20 & 0.15 & 2.41 & 0.01 \\
\hline 5 & 21.2 & 13.0 & 0.24 & 0.10 & 0.57 & -2.62 \\
\hline 6 & 9.2 & 5.6 & 0.17 & 0.12 & 1.51 & -1.19 \\
\hline 7 & 21.1 & 8.5 & 0.17 & 0.14 & 2.68 & -2.33 \\
\hline 8 & 21.2 & 8.4 & 0.13 & 0.11 & 0.26 & -2.15 \\
\hline 9 & 21.3 & 7.4 & 0.18 & 0.09 & -2.73 & 2.64 \\
\hline 10 & 21.3 & 7.0 & 0.20 & 0.09 & -1.15 & 0.30 \\
\hline 11 & 21.2 & 8.1 & 0.20 & 0.25 & -3.11 & -2.16 \\
\hline 12 & 21.1 & 5.0 & 0.19 & 0.08 & 3.11 & 2.18 \\
\hline 13 & 20.9 & 7.4 & 0.24 & 0.03 & 1.54 & 2.20 \\
\hline 14 & 20.9 & 6.2 & 0.28 & 0.10 & -0.41 & 2.61 \\
\hline 15 & 20.8 & 6.9 & 0.22 & 0.14 & -3.04 & -1.09 \\
\hline 16 & 20.9 & 8.0 & 0.17 & 0.18 & 1.09 & -2.38 \\
\hline 17 & 14.8 & 8.2 & 0.09 & 0.25 & -1.00 & 2.51 \\
\hline 18 & 8.2 & 4.8 & 0.30 & 0.36 & -0.80 & -0.36 \\
\hline
\end{tabular}

Table 5. 2 Frequencies, Magnitudes and Phases for Two Sinusoids Estimation (TUBE 1).

\begin{tabular}{|c|c|c|c|c|c|c|c|c|c|c|c|c|}
\hline $\begin{array}{c}\text { Seg } \\
\#\end{array}$ & $\begin{array}{c}\text { Frequency } \\
\text { F1(Hz) }\end{array}$ & $\begin{array}{c}\text { Frequency } \\
\text { F2(Hz) }\end{array}$ & $\begin{array}{c}\text { Frequency } \\
\text { F3(Hz) }\end{array}$ & $\begin{array}{c}\text { Frequency } \\
\text { F4(Hz) }\end{array}$ & $\begin{array}{c}\text { Magnitude } \\
\text { M1(V) }\end{array}$ & $\begin{array}{c}\text { Magnitude } \\
\text { M2(V) }\end{array}$ & $\begin{array}{c}\text { Magnitude } \\
\text { M3(V) }\end{array}$ & $\begin{array}{c}\text { Magnitude } \\
\text { M4(V) }\end{array}$ & $\begin{array}{c}\text { Phase } \\
\text { Ph1(Rad) }\end{array}$ & $\begin{array}{c}\text { Phase } \\
\text { Ph2(Rad) }\end{array}$ & $\begin{array}{c}\text { Phase } \\
\text { Ph3(Rad) }\end{array}$ & $\begin{array}{c}\text { Phase } \\
\text { Ph4(Rad) }\end{array}$ \\
\hline 1 & 21.09 & 12.05 & 8.53 & 5.47 & 0.25 & 0.15 & 0.12 & 0.06 & 2.98 & 2.65 & 1.56 & -0.19 \\
\hline 2 & 25.45 & 21.5 & 8.9 & 7.37 & 0.16 & 0.23 & 0.09 & 0.15 & -0.73 & 0.57 & 1.46 & -1.78 \\
\hline 3 & 21.06 & 12.18 & 8.31 & 4.46 & 0.16 & 0.14 & 0.08 & 0.05 & -1.31 & 1.95 & -0.18 & 2.33 \\
\hline 4 & 21.1 & 12.11 & 8.28 & 4.62 & 0.2 & 0.16 & 0.12 & 0.11 & 2.53 & 0.14 & 0.37 & 0.87 \\
\hline 5 & 21.18 & 12.03 & 8.34 & 4.65 & 0.24 & 0.17 & 0.09 & 0.09 & 0.59 & -0.56 & -0.51 & -0.64 \\
\hline 6 & 20.93 & 11.19 & 8.26 & 4.6 & 0.2 & 0.22 & 0.28 & 0.21 & 0.1 & -1.22 & -1.93 & 2.56 \\
\hline 7 & 21.06 & 12.29 & 8.23 & 3.93 & 0.16 & 0.17 & 0.11 & 0.17 & -1.39 & 2.8 & 2.76 & 1.37 \\
\hline 8 & 21.17 & 11.87 & 7.75 & 4.25 & 0.13 & 0.08 & 0.08 & 0.05 & 0.35 & 0.43 & -1.49 & -2.76 \\
\hline 9 & 21.26 & 11.86 & 8.13 & 5.06 & 0.18 & 0.11 & 0.06 & 0.09 & -2.72 & 0.43 & 2.67 & 3.1 \\
\hline 10 & 25.59 & 21.26 & 8.31 & 6.14 & 0.21 & 0.14 & 0.11 & 0.11 & -0.96 & 1.89 & 3.02 & 2.05 \\
\hline 11 & 25.72 & 21.09 & 8.16 & 4.49 & 0.19 & 0.27 & 0.12 & 0.07 & -2.87 & -2.33 & 2.36 & 2.88 \\
\hline 12 & 21.1 & 12.14 & 8.53 & 4.55 & 0.19 & 0.08 & 0.11 & 0.04 & -3.13 & 2.15 & -0.55 & -2.34 \\
\hline 13 & 20.93 & 12.13 & 8.09 & 4.98 & 0.25 & 0.07 & 0.13 & 0.09 & 1.5 & 0.33 & 0.74 & 2.07 \\
\hline 14 & 20.9 & 12.4 & 7.56 & 5.03 & 0.29 & 0.16 & 0.13 & 0.1 & -0.42 & -1.78 & -1.51 & -0.19 \\
\hline 15 & 20.8 & 12.67 & 7.8 & 4.93 & 0.21 & 0.09 & 0.09 & 0.1 & -2.97 & 0.14 & 0.06 & 1.59 \\
\hline 16 & 20.48 & 16.46 & 12.3 & 7.91 & 0.17 & 0.17 & 0.13 & 0.08 & 1.4 & -2.2 & 2.53 & -2.91 \\
\hline 17 & 16.82 & 13.43 & 7.69 & 0.08 & 0.16 & 0.18 & 0.16 & 0.01 & -1.94 & -0.01 & 3.08 & -3.14 \\
\hline 18 & 20.82 & 16.73 & 8.18 & 4.81 & 0.37 & 0.27 & 0.31 & 0.12 & -0.29 & 0.44 & -0.82 & 1.24 \\
\hline
\end{tabular}

Table 5. 3 Frequencies, Magnitudes and Phases for Four Sinusoids Estimation (TUBE 1). 


\begin{tabular}{|c|c|c|c|}
\hline Segment \# & $\begin{array}{c}\text { Frequency } \\
\text { F1(Hz) }\end{array}$ & $\begin{array}{c}\text { Magnitude } \\
\text { M1(V) }\end{array}$ & $\begin{array}{c}\text { Phase } \\
\text { Ph1(Radians) }\end{array}$ \\
\hline 1 & 6.1 & 0.03 & 1.59 \\
\hline 2 & 19.4 & 0.15 & -0.79 \\
\hline 3 & 19.3 & 0.12 & -0.08 \\
\hline 4 & 19.9 & 0.05 & -0.84 \\
\hline 5 & 6.7 & 0.17 & -1.47 \\
\hline 6 & 20.2 & 0.02 & -0.34 \\
\hline 7 & 26.0 & 0.08 & 1.08 \\
\hline 8 & 25.9 & 0.20 & -0.25 \\
\hline 9 & 26.0 & 0.31 & 0.98 \\
\hline 10 & 6.6 & 0.21 & 2.48 \\
\hline 11 & 26.1 & 0.27 & 2.80 \\
\hline 12 & 19.7 & 0.08 & 2.63 \\
\hline 13 & 19.4 & 0.06 & -1.14 \\
\hline 14 & 19.3 & 0.14 & 3.11 \\
\hline 15 & 19.1 & 0.14 & -2.34 \\
\hline 16 & 19.2 & 0.16 & -2.81 \\
\hline 17 & 6.3 & 0.10 & 1.42 \\
\hline 18 & 18.6 & 0.10 & -3.00 \\
\hline
\end{tabular}

Table 5. 4 Frequencies, Magnitudes and Phases for One Sinusoid Estimation (TUBE 2).

\begin{tabular}{|c|c|c|c|c|c|c|}
\hline $\begin{array}{c}\text { Segment } \\
\#\end{array}$ & $\begin{array}{c}\text { Frequency } \\
\text { F1(Hz) }\end{array}$ & $\begin{array}{c}\text { Frequency } \\
\text { F2(Hz) }\end{array}$ & $\begin{array}{c}\text { Magnitude } \\
\text { M1(V) }\end{array}$ & $\begin{array}{c}\text { Magnitude } \\
\text { M2(V) }\end{array}$ & $\begin{array}{c}\text { Phase } \\
\text { Ph1(Radians) }\end{array}$ & $\begin{array}{c}\text { Phase } \\
\text { Ph2(Radians) }\end{array}$ \\
\hline 1 & 19.3 & 6.2 & 0.12 & 0.03 & 2.94 & 1.99 \\
\hline 2 & 19.4 & 10.4 & 0.15 & 0.09 & -0.77 & -0.28 \\
\hline 3 & 19.3 & 8.0 & 0.11 & 0.07 & -0.11 & 1.09 \\
\hline 4 & 19.9 & 8.3 & 0.05 & 0.05 & -0.87 & 0.05 \\
\hline 5 & 8.2 & 6.4 & 0.13 & 0.18 & -0.58 & -1.14 \\
\hline 6 & 20.2 & 8.3 & 0.03 & 0.08 & -0.31 & -2.07 \\
\hline 7 & 25.8 & 20.7 & 0.08 & 0.03 & 1.68 & -1.90 \\
\hline 8 & 25.9 & 8.0 & 0.20 & 0.13 & -0.24 & -2.41 \\
\hline 9 & 26.0 & 5.3 & 0.31 & 0.13 & 0.99 & 2.48 \\
\hline 10 & 26.0 & 6.4 & 0.28 & 0.24 & -2.84 & -2.87 \\
\hline 11 & 26.1 & 7.9 & 0.27 & 0.11 & 2.80 & 0.56 \\
\hline 12 & 19.7 & 7.8 & 0.08 & 0.08 & 2.64 & 0.08 \\
\hline 13 & 19.4 & 8.3 & 0.06 & 0.07 & -1.40 & -2.16 \\
\hline 14 & 19.3 & 9.4 & 0.14 & 0.03 & 3.09 & 2.59 \\
\hline 15 & 19.1 & 7.8 & 0.14 & 0.10 & -2.46 & -2.54 \\
\hline 16 & 19.2 & 7.8 & 0.16 & 0.03 & -2.81 & 1.64 \\
\hline 17 & 19.1 & 6.6 & 0.16 & 0.10 & -1.70 & 1.46 \\
\hline 18 & 18.6 & 9.9 & 0.10 & 0.03 & -2.95 & -2.05 \\
\hline
\end{tabular}

Table 5. 5 Frequencies, Magnitudes and Phases for Two Sinusoids Estimation (TUBE 2). 


\begin{tabular}{|c|c|c|c|c|c|c|c|c|c|c|c|c|}
\hline $\begin{array}{c}\text { Seg } \\
\#\end{array}$ & $\begin{array}{c}\text { Frequency } \\
\text { F1(Hz) }\end{array}$ & $\begin{array}{c}\text { Frequency } \\
\text { F2(Hz) }\end{array}$ & $\begin{array}{c}\text { Frequency } \\
\text { F3(Hz) }\end{array}$ & $\begin{array}{c}\text { Frequency } \\
\text { F4(Hz) }\end{array}$ & $\begin{array}{c}\text { Magnitude } \\
\text { M1(V) }\end{array}$ & $\begin{array}{c}\text { Magnitude } \\
\text { M2(V) }\end{array}$ & $\begin{array}{c}\text { Magnitude } \\
\text { M3(V) }\end{array}$ & $\begin{array}{c}\text { Magnitude } \\
\text { M4(V) }\end{array}$ & $\begin{array}{c}\text { Phase } \\
\text { Ph1(Rad) }\end{array}$ & $\begin{array}{c}\text { Phase } \\
\text { Ph2(Rad) }\end{array}$ & $\begin{array}{c}\text { Phase } \\
\text { Ph3(Rad) }\end{array}$ & $\begin{array}{c}\text { Phase } \\
\text { Ph4(Rad) }\end{array}$ \\
\hline 1 & 19.3 & 11.3 & 7.3 & 4 & 0.12 & 0.13 & 0.09 & 0.06 & 2.84 & 0.06 & 2.32 & 0.49 \\
\hline 2 & 19.4 & 14 & 10.5 & 6.7 & 0.15 & 0.1 & 0.05 & 0.03 & -0.83 & -0.41 & -0.3 & 1.13 \\
\hline 3 & 25.6 & 19.3 & 9.4 & 7.5 & 0.11 & 0.07 & 0.06 & 0.01 & -0.04 & 0.06 & 2.67 & 0.39 \\
\hline 4 & 26 & 19.8 & 10.3 & 7 & 0.06 & 0.07 & 0.02 & 0.03 & -0.56 & -1.93 & -2.99 & -2.63 \\
\hline 5 & 25.7 & 12.9 & 8.8 & 6.5 & 0.16 & 0.08 & 0.1 & 0.06 & -1.24 & -2.84 & -1.16 & -0.97 \\
\hline 6 & 26.3 & 20.2 & 10.8 & 8 & 0.02 & 0.08 & 0.04 & 0.04 & -0.3 & -1.29 & 1.3 & 1.38 \\
\hline 7 & 31.4 & 25.8 & 20.8 & 8.1 & 0.08 & 0.04 & 0.06 & 0.01 & 1.69 & -2.1 & -2.02 & 1.74 \\
\hline 8 & 25.9 & 19.3 & 7.3 & 1.4 & 0.2 & 0.03 & 0.07 & 0.08 & -0.22 & -2.76 & 0.45 & 0.43 \\
\hline 9 & 26 & 21.2 & 10.1 & 5.3 & 0.31 & 0.12 & 0.05 & 0.05 & 1.02 & 2.27 & -3.14 & 0.84 \\
\hline 10 & 26 & 11.9 & 9.7 & 6.2 & 0.28 & 0.24 & 0.11 & 0.04 & -2.84 & -2.38 & -2.17 & -0.07 \\
\hline 11 & 26.1 & 20.5 & 9.6 & 6.1 & 0.27 & 0.05 & 0.01 & 0.05 & 2.8 & -1.59 & -2.43 & -1.41 \\
\hline 12 & 26.1 & 19.7 & 7.5 & 4 & 0.09 & 0.07 & 0.07 & 0.05 & 2.64 & 0.38 & 0.89 & -2.11 \\
\hline 13 & 19.6 & 10 & 9 & 0.001 & 0.06 & 0.01 & 0.06 & 0.02 & -2.36 & -3.14 & 2.72 & 1.21 \\
\hline 14 & 22.7 & 19.3 & 9.3 & 4.5 & 0.14 & 0.05 & 0.02 & 0.05 & -3.11 & -1.2 & 2.67 & -0.02 \\
\hline 15 & 22.7 & 19.2 & 7.8 & 6.6 & 0.13 & 0.1 & 0.05 & 0.05 & -2.58 & -2.69 & 2.28 & -0.14 \\
\hline 16 & 23.4 & 19.2 & 11.1 & 7.1 & 0.16 & 0.06 & 0.03 & 0.02 & -2.81 & -2.99 & -0.44 & 0.66 \\
\hline 17 & 19 & 10.2 & 6.3 & 5 & 0.16 & 0.1 & 0.04 & 0.08 & -1.68 & 1.33 & 2.04 & 2.93 \\
\hline 18 & 18.7 & 12.9 & 9.6 & 6.4 & 0.11 & 0.04 & 0.09 & 0.06 & 3.03 & -2.87 & 1.82 & -1.47 \\
\hline
\end{tabular}

Table 5. 6 Frequencies, Magnitudes and Phases for Four Sinusoids Estimation (TUBE 2).

\subsubsection{Improved Estimation using Rectangular Windowing}

Some of the segments in TUBE 1 contain peaks ${ }^{22}$ which affect the estimation process.

From Table 5.2, we notice that segment 6,17 and 18 have different dominant frequency from other segments. In addition, these segments have peaks as shown in Figure5.3.

The peak to average ratio is defined as follows equation (28):

$$
P A R=\frac{X_{p}}{\sqrt{\frac{\sum_{i=1}^{N}\left(X_{i}-m\right)^{2}}{N}}}
$$

\footnotetext{
${ }^{22}$ These peaks maybe caused by noise impulses, flaw responses and external interference.
} 
where

$X_{p}$ is the magnitude of the peak

$X_{i}$ is the element of the segment from $i=1 \cdots \cdots \cdots N$

$m$ is the mean of the segment

$N$ is the number of data samples

The peak to average ratio PAR determines how extreme the peaks are in segments. By Trial and error method it was found that segments with peak to average ratio greater than $5 \mathrm{~dB}$ could have improved estimates through the use of (window ${ }^{23}$ ) function, segment 6 and 17 in TUBE 1 satisfy the PAR condition.

By truncating the peaks and applying a rectangular window to the segment, we can improve the frequency estimation which results in better sinusoidal interference removal. In this process, we are considering two methods:

1- Excluding the peak from the window segment

2- Replace the truncated peak only with the average of the segment.
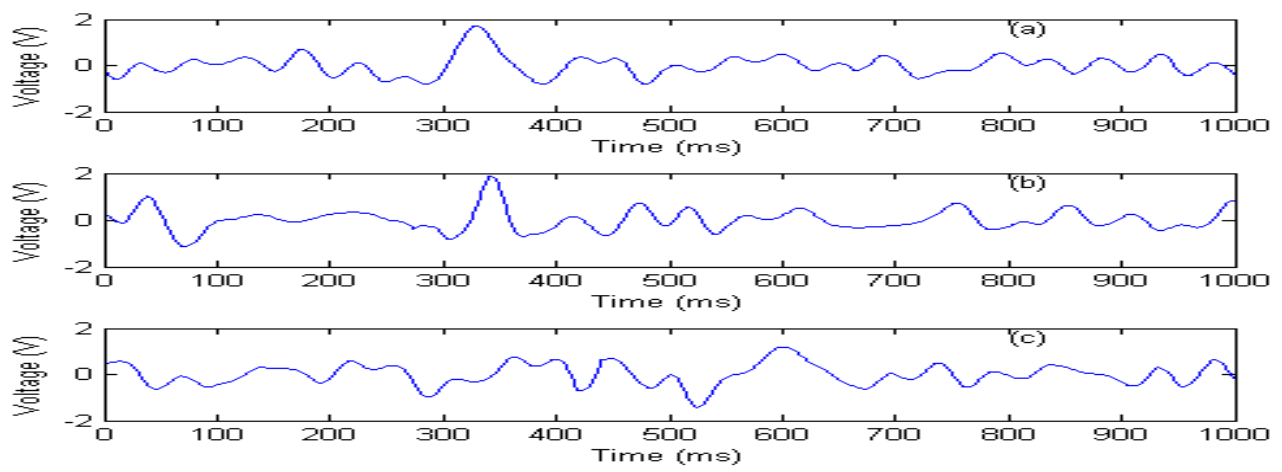

Figure 5.3 a) Segment 6 b) Segment 17 c) Segment 18 (TUBE 1).

${ }^{23}$ This is not the conventional window function. 
Applying the two methods to segment 6, the first method truncates the first part of the segment where the peak is and replaces it by zeros and applies a rectangular window to the second part of the segment starting from point 500 to 1000 . The second method truncates only the peaks and replaces it by the average of the segment and windows the rest of the segment. Figure 5.4 shows the original segment and the two methods.

The estimation of the two methods has showed an improvement compared to the original estimation and this can be shown in Figure5.5. From Figure5.5, we notice that method two (truncate peak and replace by average of the segment) has showed better performance than the first method (excluding peak from window segment). Table 5.7, shows the improved frequency estimations for segment 6 and 17 for the second method.
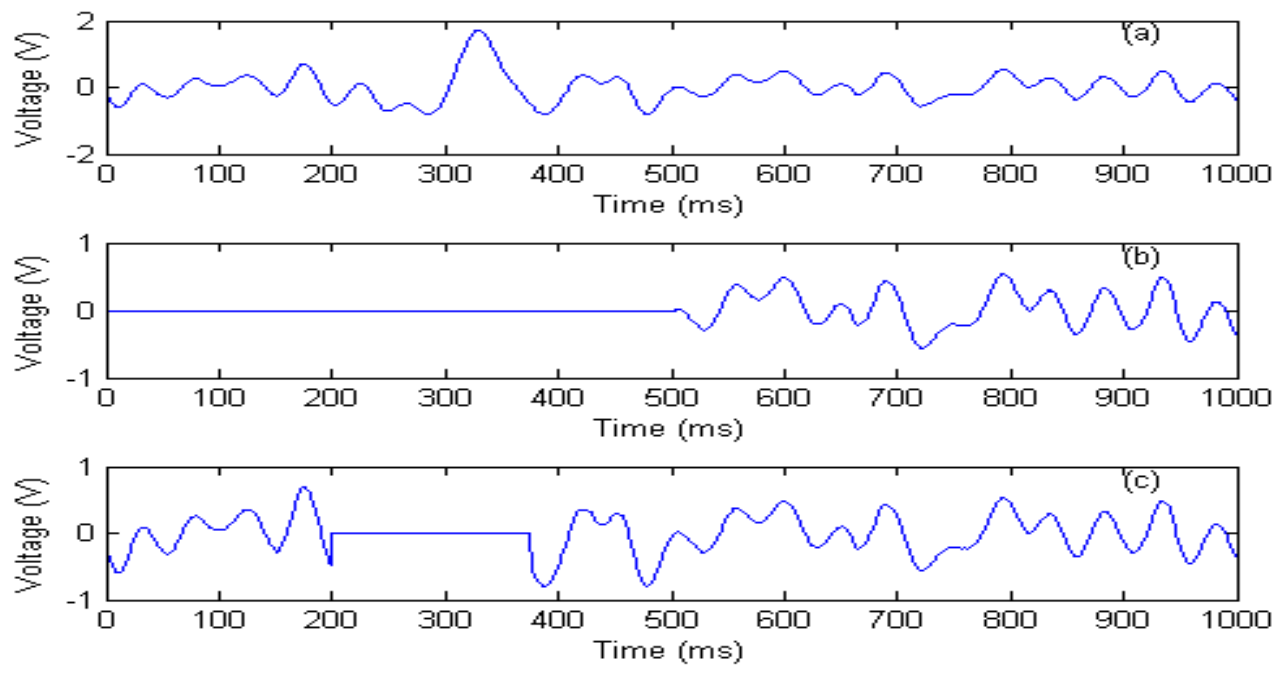

Figure 5. 4 a) Original Segment b) Excluding Peak Method c) Truncating Peak Method (TUBE 1).

\begin{tabular}{|c|c|c|c|c|}
\hline Segment \# & $\begin{array}{c}\text { Original } \\
\text { estimation } \\
\text { F1(Hz) }\end{array}$ & $\begin{array}{c}\text { Improved Frequency } \\
\text { estimation F1(Hz) }\end{array}$ & $\begin{array}{c}\text { Original } \\
\text { estimation } \\
\text { F2(Hz) }\end{array}$ & $\begin{array}{c}\text { Improved Frequency } \\
\text { estimation F2 (Hz) }\end{array}$ \\
\hline 6 & 9.2 & 20.9 & 5.6 & 4.7 \\
\hline 17 & 14.8 & 19.7 & 8.2 & 8.4 \\
\hline
\end{tabular}

Table 5. 7 Improved Frequency Estimation for Two Sinusoids. 

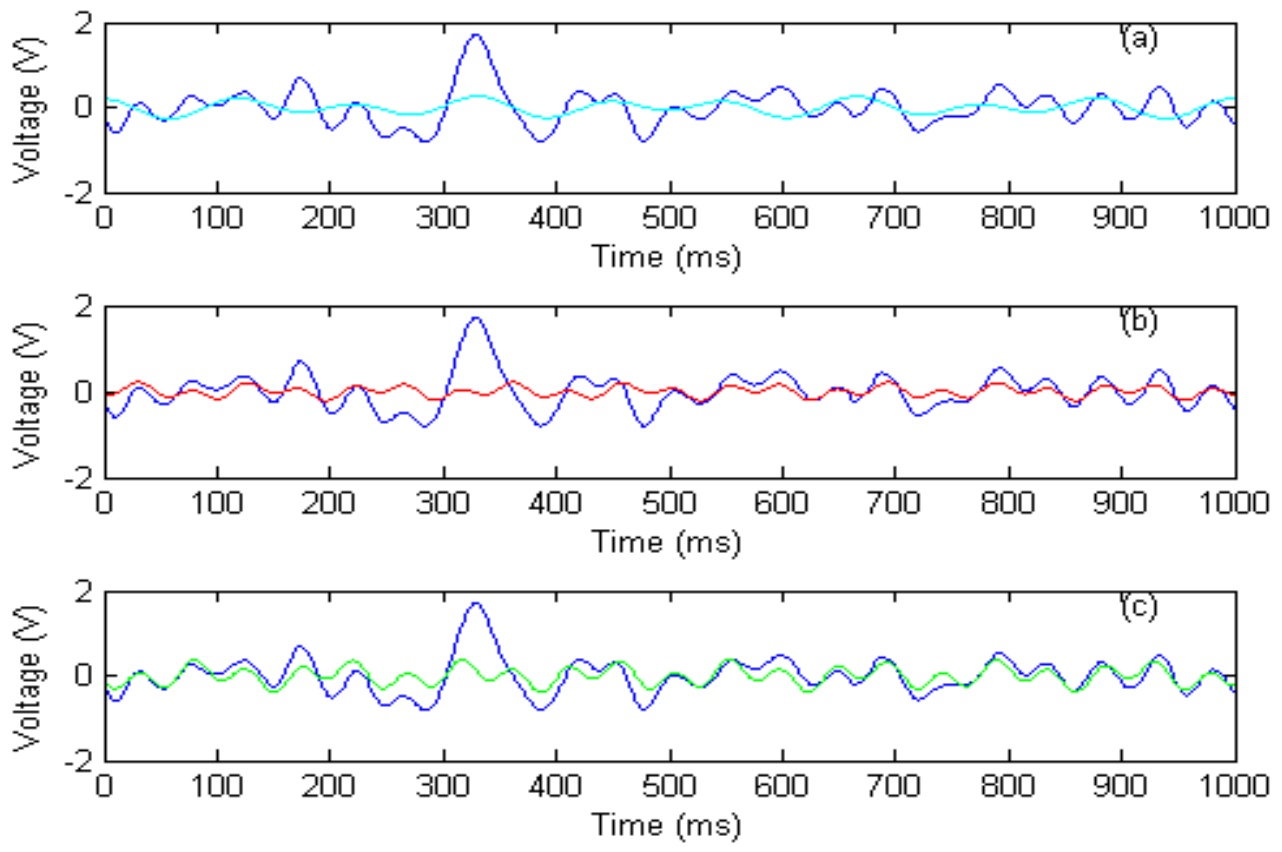

Figure 5. 5 Original Segment and Estimated Waveform for a) Original Estimation b) Excluding Peak Method c) Truncating Peak Method (TUBE 1).

\subsection{Sinusoidal Interference Removal}

The low frequency sinusoidal interference removal process consists of two steps. First, generate the sinusoidal waveforms using the estimated parameters and subtract the waveforms from the original segment.

Three removal methods are considered, using all estimated parameters for each segment, use tube average frequency for each segment and use tube average frequency and average magnitude for the whole tube data record.

\subsubsection{Using all Estimated Sinusoidal Parameters}

Using the estimated frequency, magnitude and phase for each segment to generate the sinusoidal waveform and subtract it from the original segment. Figures 5.6 to 5.11 show removal process of one, 
two and four sinusoids for segment 15 from TUBE 1 data record and segment 14 from TUBE 2 data record. The $\mathrm{X}$-axis is time and the $\mathrm{Y}$-axis is amplitude.
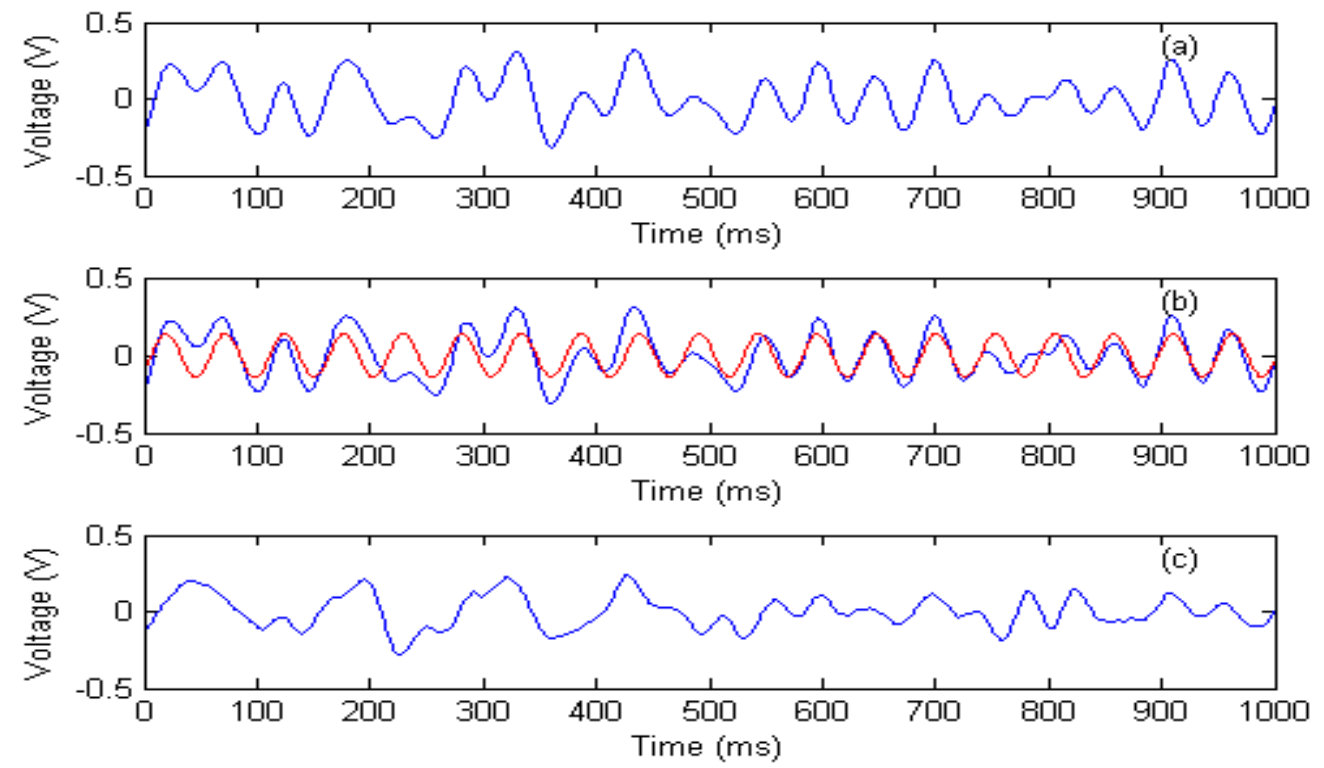

Figure 5. 6 Removal Process For One Sinusoid a) Original Segment b) Original Segment and Estimated Waveform, c) Subtraction Result (TUBE 1).
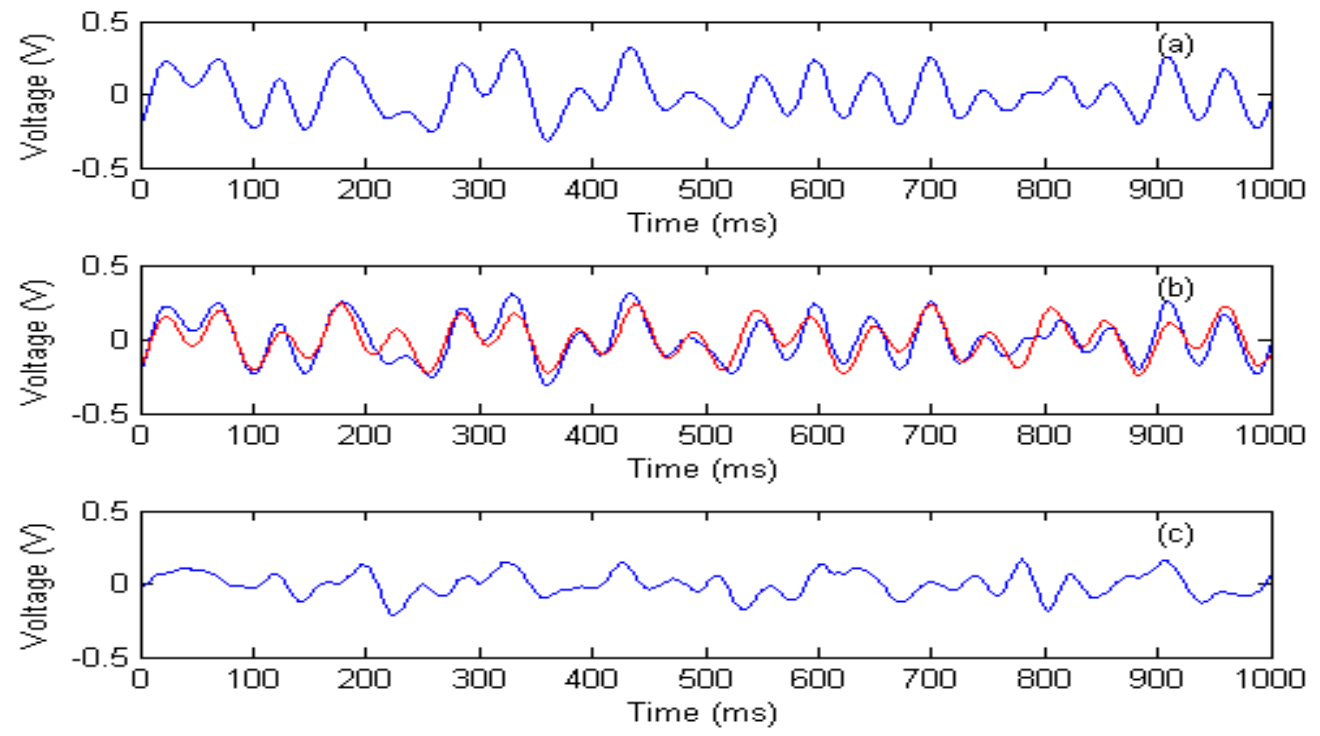

Figure 5. 7 Removal Process for Two Sinusoids a) Original Segment b) Original Segment and Estimated Waveform, c) Subtraction Result (TUBE 1). 

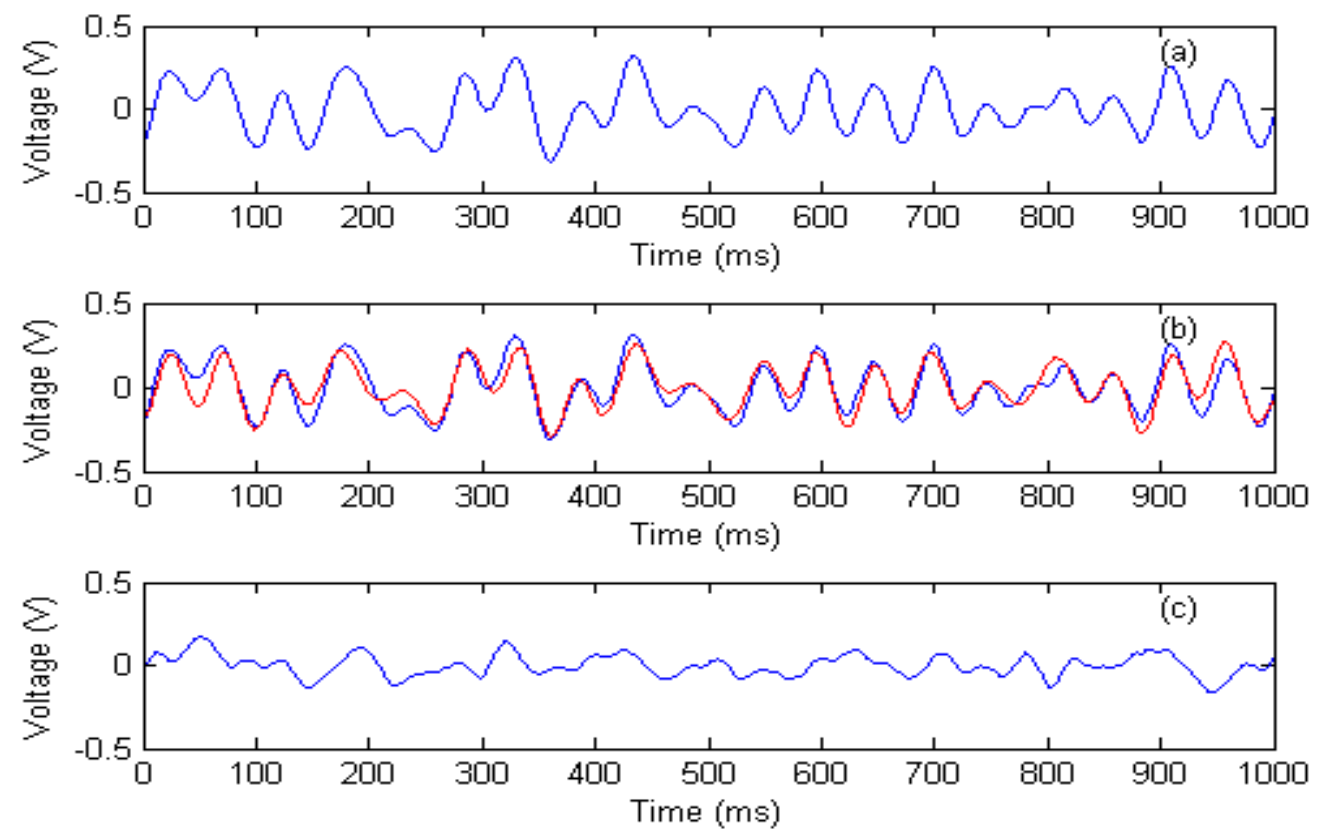

Figure 5. 8 Removal Process for Four Sinusoids a) Original Segment b) Original Segment and Estimated Waveform, c) Subtraction Result (TUBE 1).
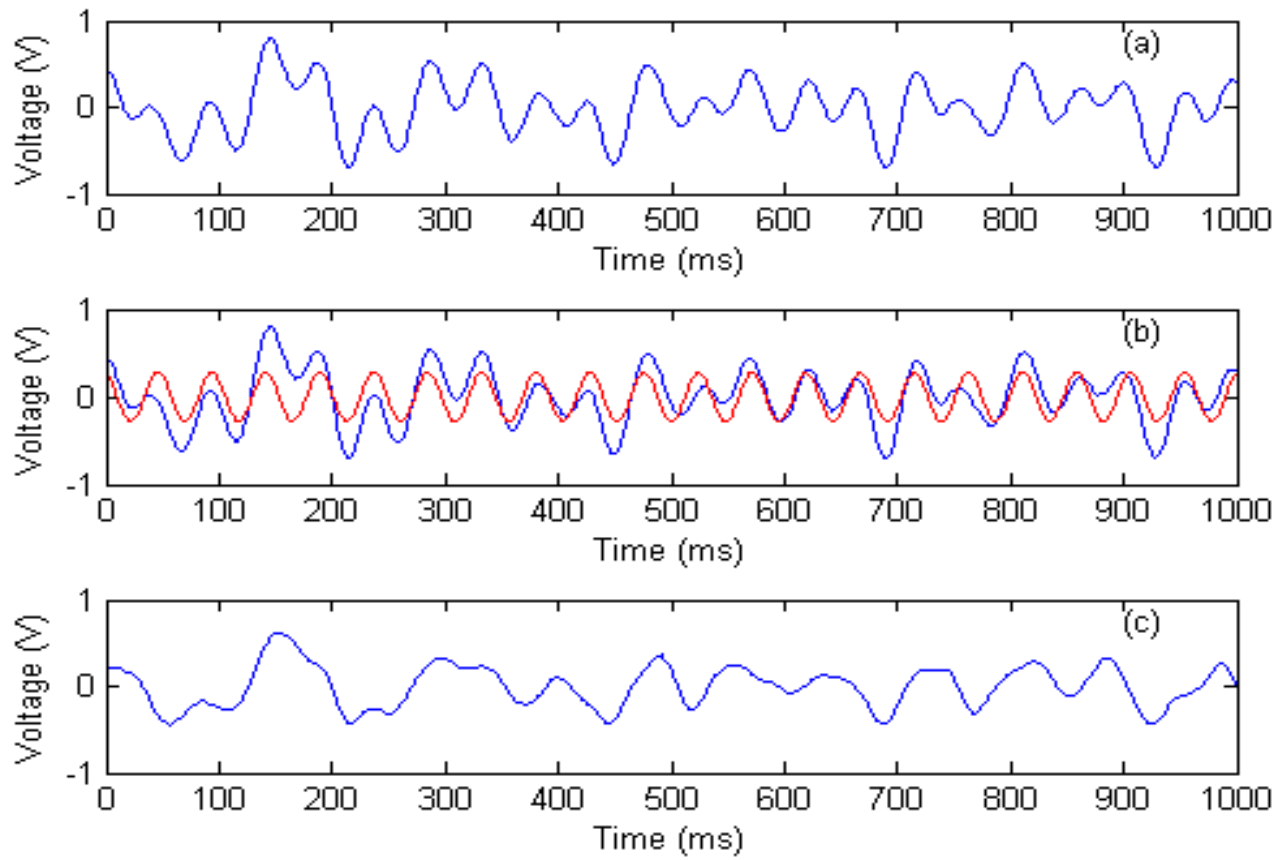

Figure 5. 9 Removal Process for One Sinusoid a) Original Segment b) Original Segment and Estimated Waveform, c) Subtraction Result (TUBE 2). 

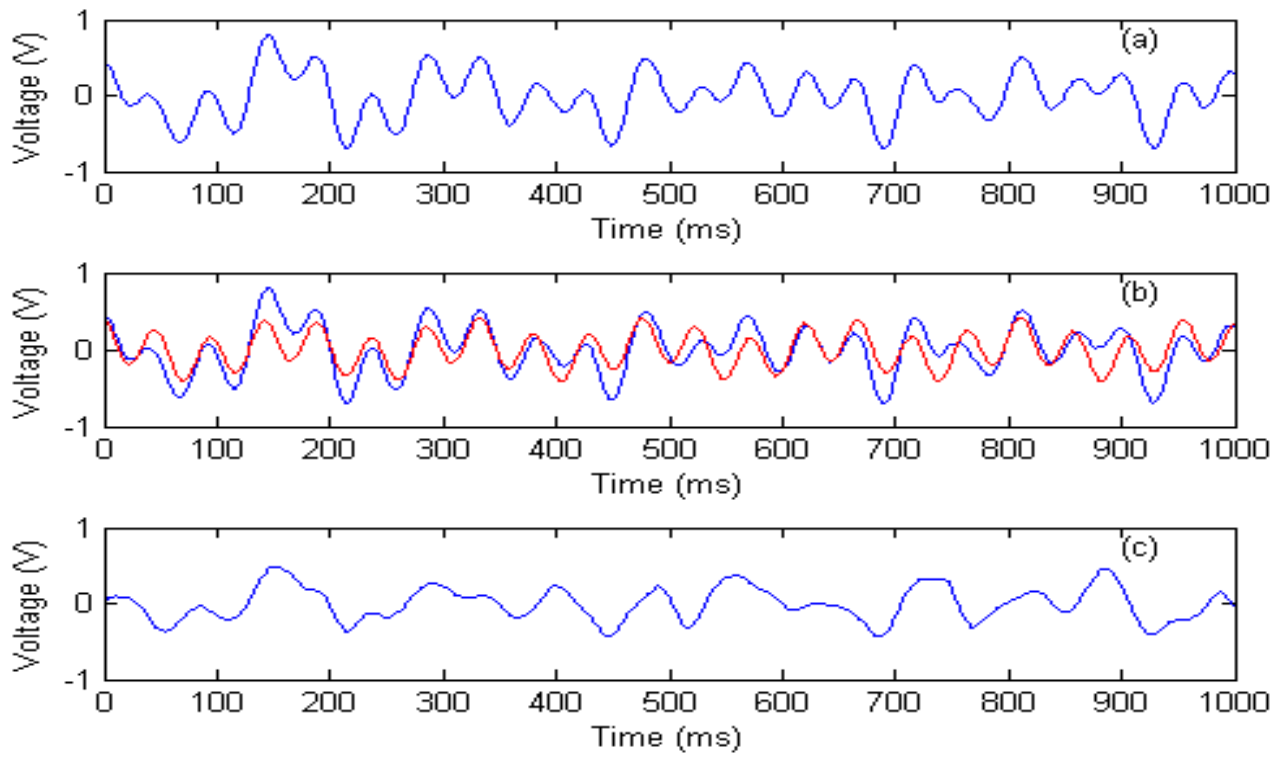

Figure 5. 10 Removal Process for Two Sinusoids a) Original Segment b) Original Segment and Estimated Waveform, c) Subtraction Result (TUBE 2).
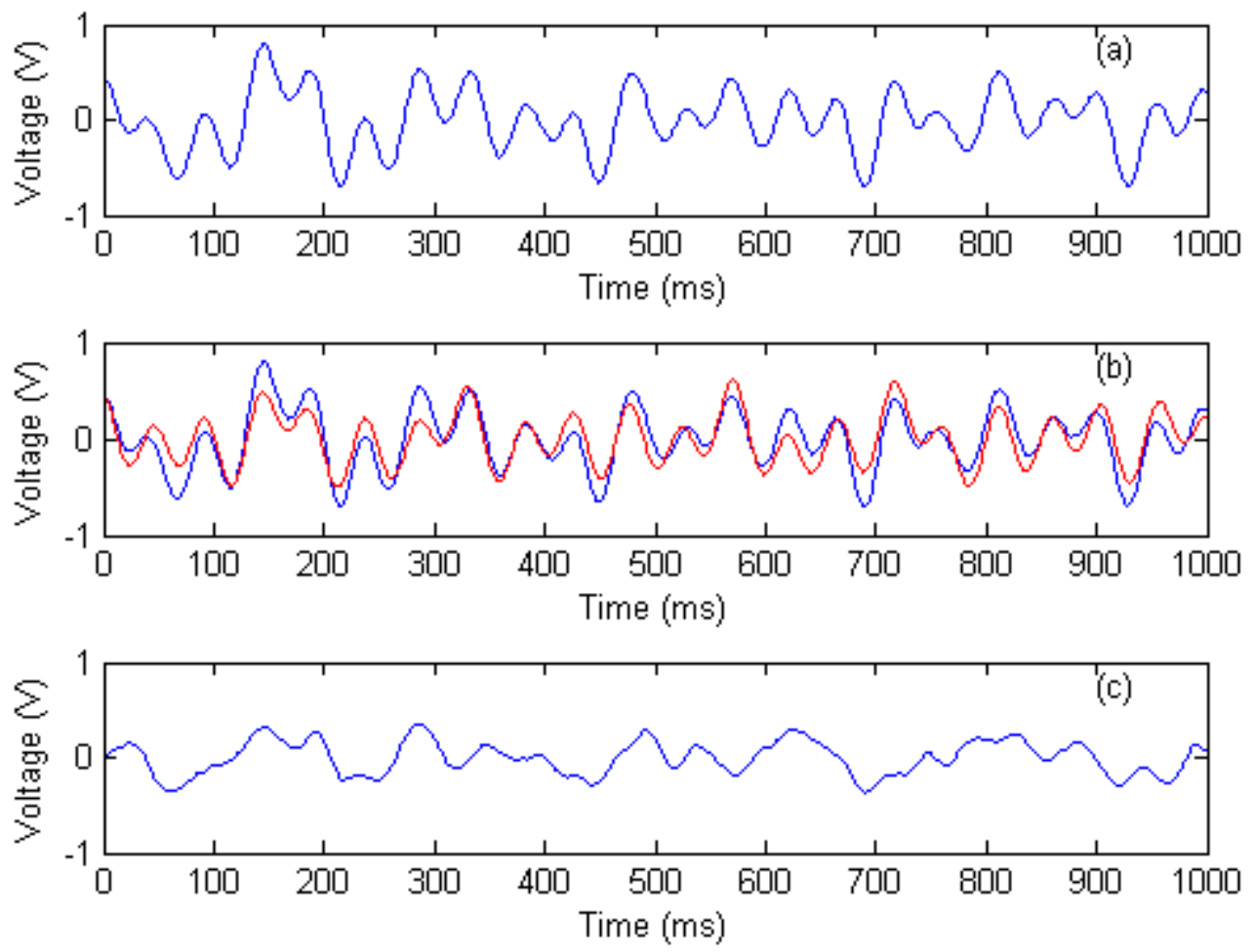

Figure 5. 11 Removal Process for Four Sinusoids a) Original Segment b) Original Segment and Estimated Waveform, c) Subtraction Result (TUBE 2). 
As shown in figures 5.6 and 5.9, we observe that removing one sinusoid from the data segments does not eliminate all the sinusoidal interference; some sinusoidal patterns are still shown in the results. On the other hand, removing two sinusoids from the data segments as shown in figures 5.7 and 5.10 preserved some features in the data segments while capturing most of the sinusoidal interference. In addition, removing four sinusoids from the data segments as shown in figures 5.8 and 5.11 eliminated all the features in the data segments. Power and correlation analysis are discussed in section 5.3 and 5.4.

\subsubsection{Using the Tube Average Frequency for Each Segment}

This method deals with averaging the estimated frequencies (two sinusoids estimation) for all segments in the tube and applying it to each segment while using the estimated magnitude and phase for each segment. Some segments might have interference from other sources in the tube, for instance mechanical noise or vibration which causes fluctuation in frequencies, as shown in figures 5.1 and 5.2. This case is shown in Figure5.12. As a result, the average frequency method has added more sinusoidal noise to the data segments.
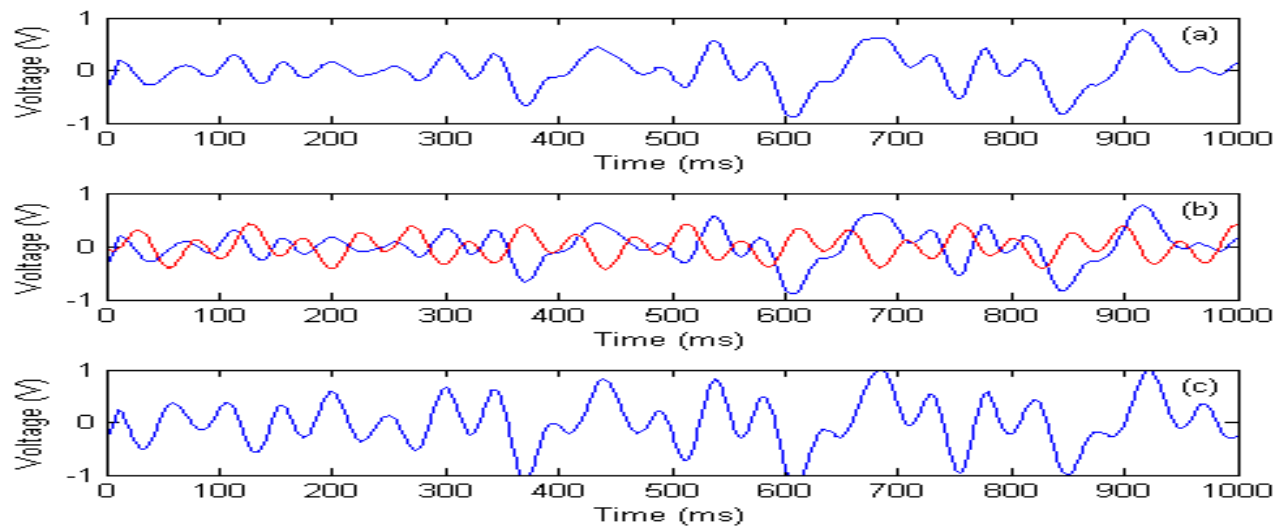

Figure 5. 12 Tube Average Frequency Technique a) Original Segment b) Original Segment and Estimated Waveform, c) Subtraction Result (TUBE 1). 


\subsubsection{Using Tube Average Frequency and Magnitude for the Whole}

\section{Data Record}

The third method deals with averaging the frequencies and magnitudes for all segments in the tube and applying it for the tube data record. This approach is useful when we know that the tube is suffering from steady noise with steady amplitude. In our case, the frequency and magnitude fluctuates from segment to segment. Figures 5.13 and 5.14 show the fluctuation of magnitudes for all segments for the two sinusoidal noise estimations.

In addition, support plates responses in the data record affects the removal process. Figure 5.15 shows the first 2500 points of the data record and the averaged frequency and magnitude waveform; we notice a phase shift in the waveform at location 1300 where the support plates are. As a result, more sinusoidal noise is added to the whole data record.

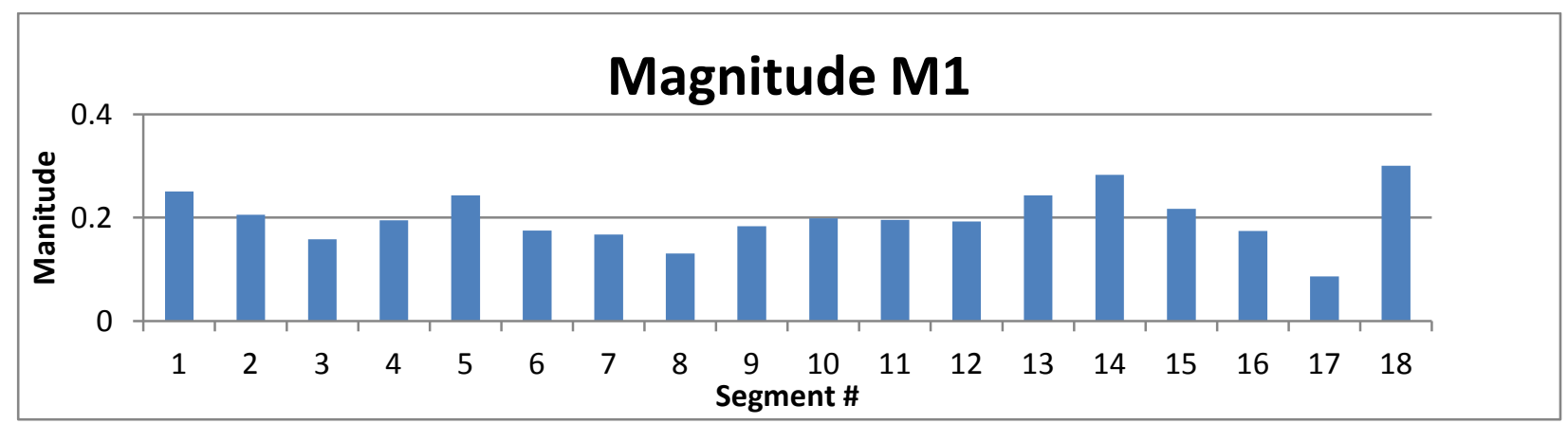

Figure 5. 13 Bar Plot for The Magnitude of First Frequency.

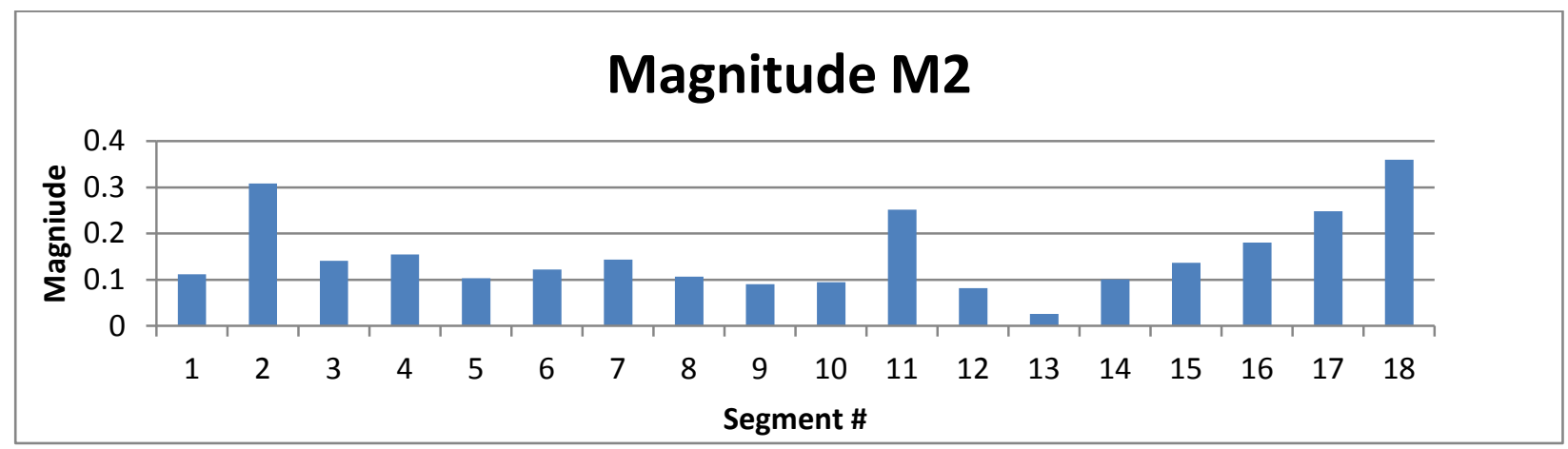

Figure 5. 14 Bar Plot for The Magnitude of Second Frequency. 

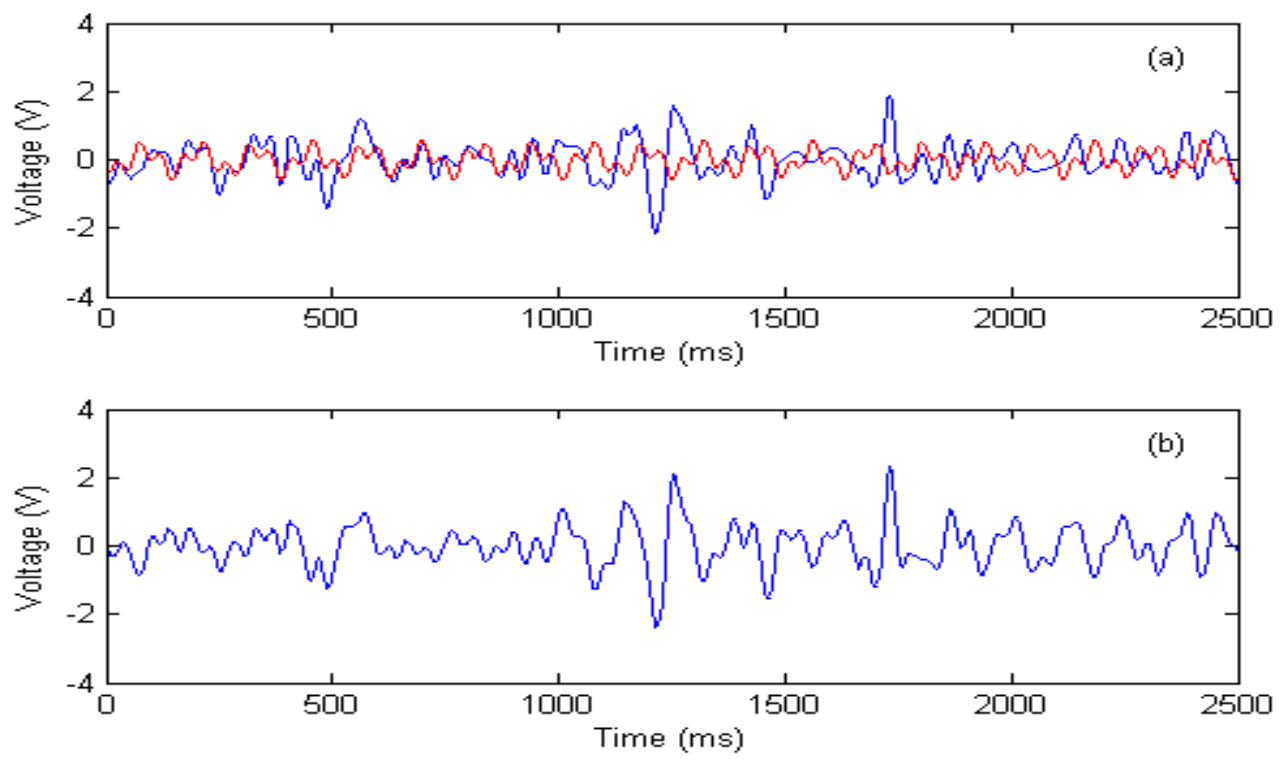

Figure 5. 15 Tube Average Frequency and Magnitude a) Original Data Record and Estimated Waveform, c) Subtraction Result (TUBE 1).

\subsection{Mean Square Analysis}

The mean square method measures the change in power of the signal before and after the removal of the low frequency sinusoidal noise from the data segments. First step, calculate the mean square (power) for original data segments. For all sinusoidal estimation, generate the waveforms from the estimated sinusoidal parameters and subtract it from the original data segments, then taking the mean square of the resultant. Table 5.8 shows the mean square of the original data segments and the mean square after the removal of one, two and four sinusoids and the percentage of change in power using all the estimated sinusoidal parameters. Similarly, Table 5.9 shows the mean square after the removal of two sinusoids using tube average frequency for each segment and power change percentage. Also table 5.10 shows the improved power for segments 6 and17. 


\begin{tabular}{|c|c|c|c|c|c|c|c|}
\hline $\begin{array}{c}\text { Segment } \\
\#\end{array}$ & $\begin{array}{c}\text { Original } \\
\text { Power of each } \\
\text { segment }\end{array}$ & $\begin{array}{c}\text { Power after 1 } \\
\text { sinusoid } \\
\text { removal }\end{array}$ & $\begin{array}{c}\% \text { change } \\
\text { Reduction }\end{array}$ & $\begin{array}{c}\text { Power after 2 } \\
\text { sinusoid } \\
\text { removal }\end{array}$ & $\begin{array}{c}\% \text { change } \\
\text { Reduction }\end{array}$ & $\begin{array}{c}\text { Power } \\
\text { after 4 } \\
\text { sinusoid } \\
\text { removal }\end{array}$ & $\begin{array}{c}\% \text { change } \\
\text { Reduction }\end{array}$ \\
\hline 1 & 0.045 & 0.024 & $47 \%$ & 0.020 & $56 \%$ & 0.013 & $72 \%$ \\
\hline 2 & 0.073 & 0.054 & $27 \%$ & 0.037 & $50 \%$ & 0.056 & $22 \%$ \\
\hline 3 & 0.024 & 0.018 & $28 \%$ & 0.010 & $58 \%$ & 0.006 & $74 \%$ \\
\hline 4 & 0.033 & 0.023 & $30 \%$ & 0.016 & $52 \%$ & 0.006 & $79 \%$ \\
\hline 5 & 0.046 & 0.024 & $48 \%$ & 0.022 & $53 \%$ & 0.007 & $80 \%$ \\
\hline 6 & 0.100 & 0.080 & $20 \%$ & 0.094 & $6 \%$ & 0.039 & $61 \%$ \\
\hline 7 & 0.043 & 0.035 & $17 \%$ & 0.028 & $34 \%$ & 0.011 & $75 \%$ \\
\hline 8 & 0.022 & 0.019 & $12 \%$ & 0.014 & $39 \%$ & 0.014 & $39 \%$ \\
\hline 9 & 0.036 & 0.027 & $26 \%$ & 0.024 & $33 \%$ & 0.021 & $53 \%$ \\
\hline 10 & 0.053 & 0.042 & $22 \%$ & 0.038 & $28 \%$ & 0.020 & $57 \%$ \\
\hline 11 & 0.060 & 0.044 & $27 \%$ & 0.035 & $41 \%$ & 0.017 & $68 \%$ \\
\hline 12 & 0.034 & 0.024 & $30 \%$ & 0.020 & $43 \%$ & 0.016 & $53 \%$ \\
\hline 13 & 0.046 & 0.028 & $40 \%$ & 0.026 & $43 \%$ & 0.027 & $48 \%$ \\
\hline 14 & 0.061 & 0.040 & $35 \%$ & 0.036 & $42 \%$ & 0.022 & $63 \%$ \\
\hline 15 & 0.047 & 0.035 & $26 \%$ & 0.024 & $48 \%$ & 0.026 & $44 \%$ \\
\hline 16 & 0.048 & 0.043 & $11 \%$ & 0.034 & $30 \%$ & 0.037 & $33 \%$ \\
\hline 17 & 0.109 & 0.087 & $20 \%$ & 0.084 & $23 \%$ & 0.091 & $21 \%$ \\
\hline 18 & 0.131 & 0.126 & $4 \%$ & 0.070 & $47 \%$ & 0.036 & $72 \%$ \\
\hline
\end{tabular}

Table 5. 8 Mean Square Using All Estimated Parameters for One, Two and Four Sinusoids (TUBE 1).

\begin{tabular}{|c|c|c|c|}
\hline $\begin{array}{c}\text { Segment } \\
\#\end{array}$ & $\begin{array}{c}\text { Original } \\
\text { Power of } \\
\text { segment }\end{array}$ & $\begin{array}{c}\text { Power after 2 } \\
\text { sinusoid removal }\end{array}$ & $\%$ change \\
\hline 1 & 0.045 & 0.037 & $-19 \%$ \\
\hline 2 & 0.073 & 0.079 & $+7 \%$ \\
\hline 3 & 0.024 & 0.014 & $-44 \%$ \\
\hline 4 & 0.033 & 0.024 & $-26 \%$ \\
\hline 5 & 0.046 & 0.031 & $-31 \%$ \\
\hline 6 & 0.100 & 0.109 & $+9 \%$ \\
\hline 7 & 0.043 & 0.059 & $+40 \%$ \\
\hline 8 & 0.022 & 0.024 & $+9 \%$ \\
\hline 9 & 0.036 & 0.033 & $-9 \%$ \\
\hline 10 & 0.053 & 0.047 & $-12 \%$ \\
\hline 11 & 0.060 & 0.136 & $+127 \%$ \\
\hline 12 & 0.034 & 0.031 & $-11 \%$ \\
\hline 13 & 0.046 & 0.030 & $-36 \%$ \\
\hline 14 & 0.061 & 0.043 & $-29 \%$ \\
\hline 15 & 0.047 & 0.025 & $-48 \%$ \\
\hline 16 & 0.048 & 0.053 & $+9 \%$ \\
\hline 17 & 0.109 & 0.107 & $-2 \%$ \\
\hline 18 & 0.131 & 0.102 & $-3 \%$ \\
\hline
\end{tabular}

Table 5. 9 Mean Square Using Tube Average Frequency for Two Sinusoids (TUBE 1). 


\begin{tabular}{|c|c|c|c|}
\hline Segment \# & Original segment Power & $\begin{array}{c}\text { Improved Power for two } \\
\text { sinusoid }\end{array}$ & \% change \\
\hline 6 & 0.100 & 0.069 & $31 \%$ \\
\hline 17 & 0.109 & 0.056 & $49 \%$ \\
\hline
\end{tabular}

Table 5. 10 Improved Power for the Truncated Peak Method.

We observe that using the tube average frequency method has increased the power in most of the segments by at least $2 \%$, this is due to the fact that the dominant frequency of the segment does not match the averaged one. The consequence of averaging the frequency is more sinusoidal noise is added to the original segment. On the other hand, using all the estimated sinusoidal parameters decreased the power after removing one, two and four sinusoids by at least $4 \%, 6 \%$ and $21 \%$ respectively. Also we notice an improvement in power for segments 6 and 17 after using the truncated peak method, for instance the power reduction in segments 6 and 17 went down from $6 \%$ to $31 \%$ and from $2 \%$ to $49 \%$ respectively. In fact, using all the estimated sinusoidal parameters and the truncated peak method are the best fit for removing one and two sinusoidal interference from the data segments.

\subsection{Correlation Analysis}

Correlation analysis is the measure of change of the signal before and after the removal of the low-frequency sinusoidal noise. As stated before, the goal is to remove the low-frequency sinusoidal noise while attempting to preserve all the features of the flaw signal. The correlation process starts by correlating the flaw signal with itself and then correlates the flaw signal with the result after removing one sinusoid, two sinusoids and four sinusoids. Figure 5.16 shows the flaw signal before and after the removal of one sinusoid, 2 sinusoids and 4 sinusoids using all estimated sinusoidal parameters. From Figure 5.16, we observe a slight change in the flaw signal 
after remove one, two and four sinusoids. In contrast, Figure 5.17 shows the flaw signal before and after the removal of two sinusoids using the tube average method; we notice a significant change in the flaw signal after removing the two sinusoids. Note that in this analysis the location of the flaw signals must be identified.

The correlation coefficients using all estimated parameters have the highest correlation with original flaw signal compared to using the tube average frequency. The results are shown in table 5.11 .

\begin{tabular}{|c|c|c|c|c|}
\hline & Autocorrelation & Xcorr (1 sinusoid) & Xcorr(2 sinusoids) & Xcorr (4 sinusoids) \\
\hline $\begin{array}{c}\text { All estimated } \\
\text { Parameters }\end{array}$ & 1 & 0.9980 & 0.9983 & 0.9557 \\
\hline $\begin{array}{c}\text { Tube average } \\
\text { frequency }\end{array}$ & 1 & & 0.9488 & \\
\hline
\end{tabular}

Table 5. 11 Correlation Coefficients Before and After the Removal of Low Frequency Sinusoidal Noise Using all Estimated Parameters and Tube Average Frequency Techniques.
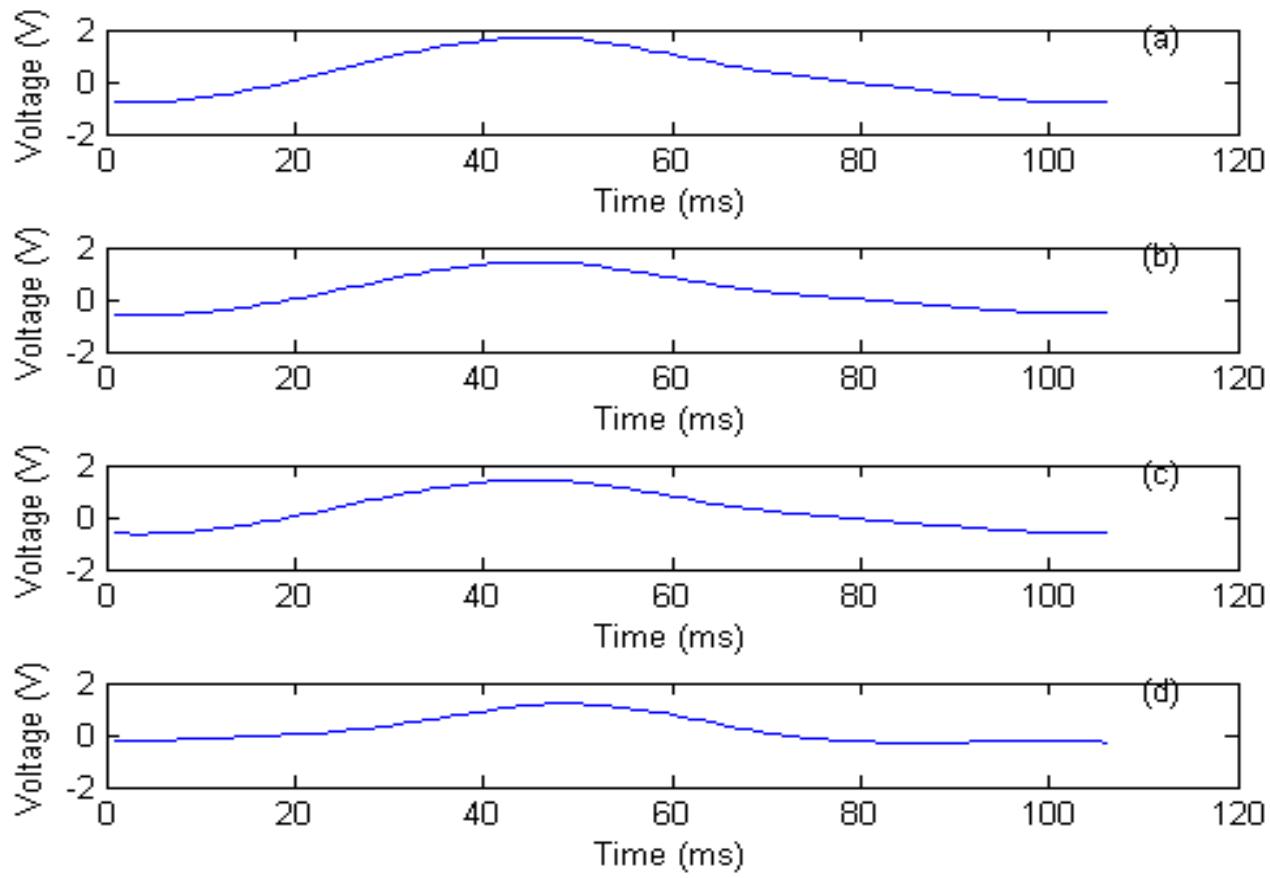

Figure 5. 16 Before and After the Removal Of Sinusoids Using All Estimated Parameters a) Flaw Signal b) One Sinusoid c) Two Sinusoids And d) Four Sinusoids (TUBE 1). 

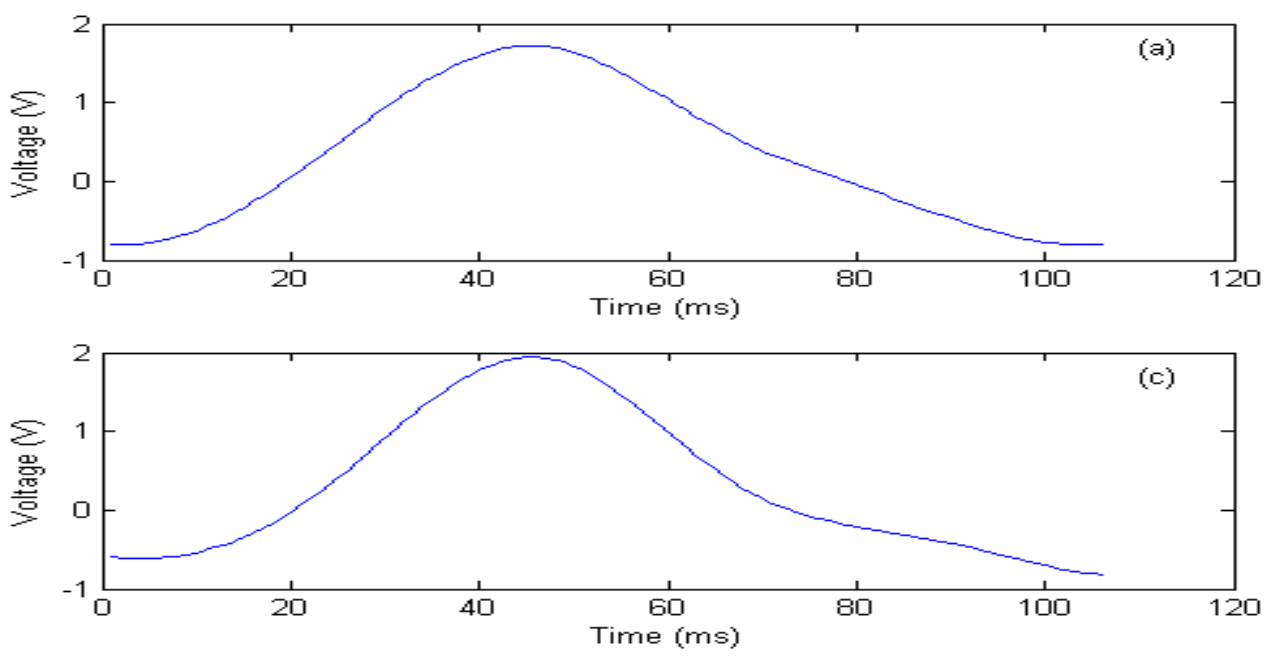

Figure 5. 17 Before and After the Removal Of Sinusoids Using Tube Average Frequency Technique a) Flaw Signal b) Two Sinusoids (TUBE 1).

\subsection{Residuals Analysis}

Residuals are the leftovers from the signal after removing the low-frequency sinusoidal noise. Examining Residuals is a key part of all statistical models and can be thought of as a source of variation in the fitted model. In our case, we are removing the estimated sinusoids from the original data and checking the model of the residuals. Figure 5.18 shows the histogram of the data before and after removing one and two sinusoidal from the signal.

Q-Q plot is a plot of the percentiles of a standard normal distribution against the corresponding percentiles of the observed data. If the observations follow approximately a normal distribution, the resulting plot should be roughly a straight line with a positive slope.

Figure 5.18 reveals that the model of the data is close to normal distribution. Using the QQ plot analysis, Figure5.19 shows the QQ plots before and after the removal of the low frequency sinusoidal interference, we notice that QQ plots are straight line in the middle and tails are off 
line. This is a good indication that the data model is close to normal, and the data is suffering from some abnormalities due to fluctuating frequencies and magnitudes in the original data.
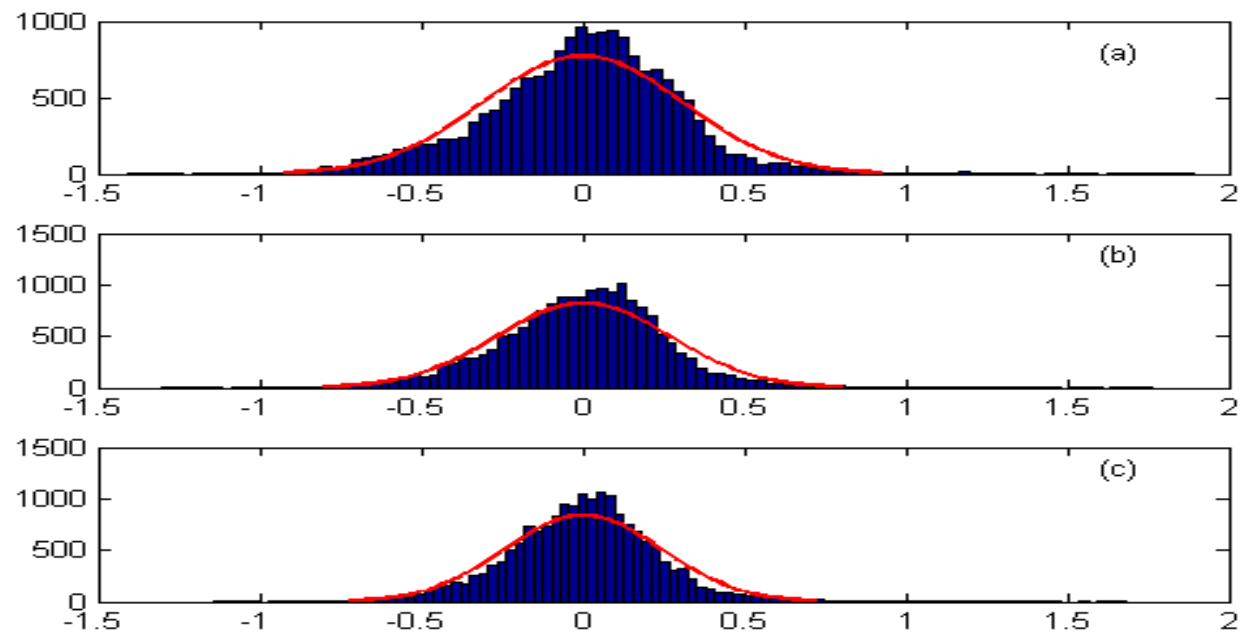

Figure 5.18 a) Histogram of All Segments and Residuals After Removing b) One Sinusoid c) Two Sinusoids (TUBE 1).

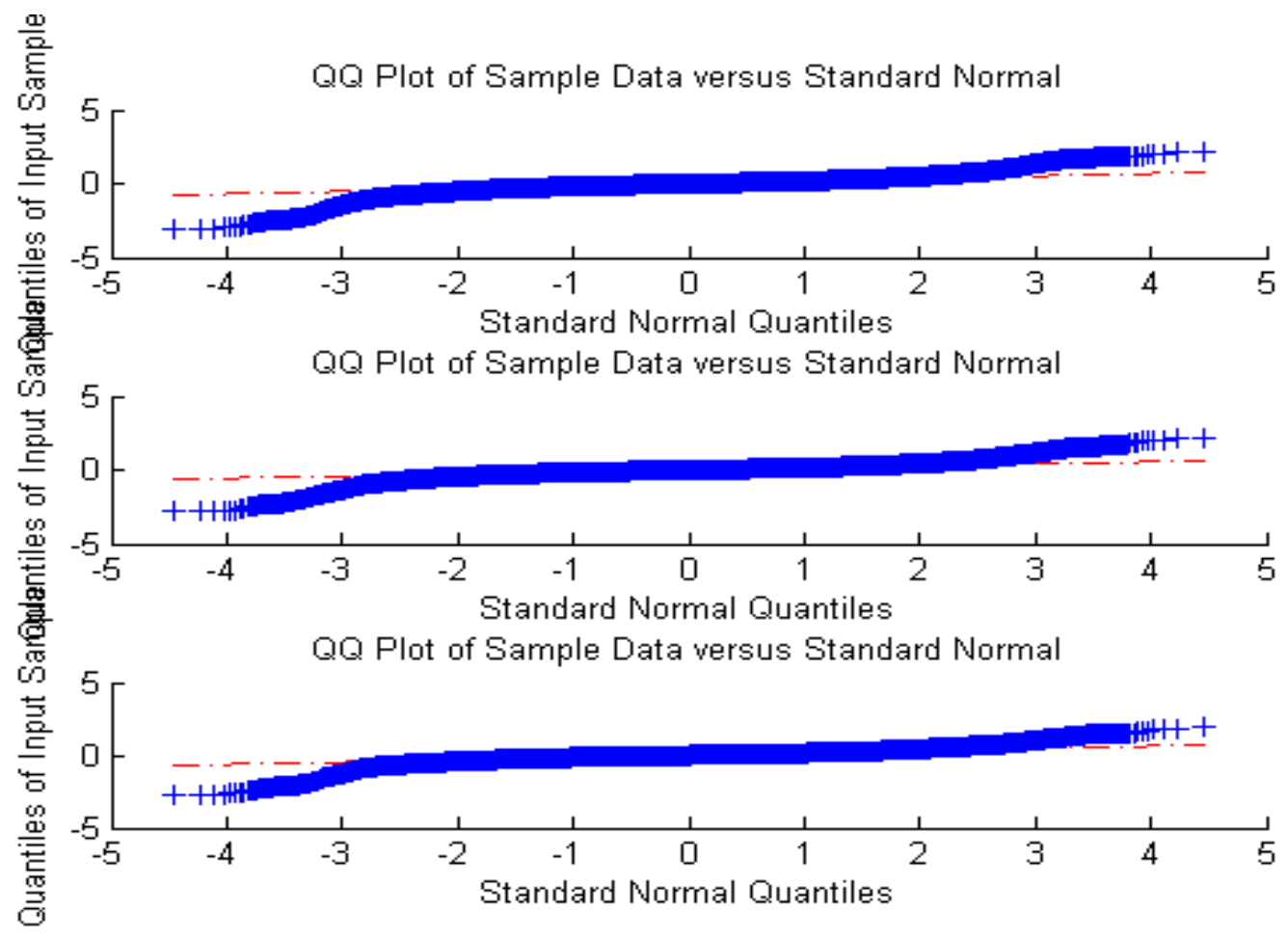

Figure 5. 19 a) QQ Plot of the Original Data b) QQ Plot After Removing One Sinusoid c) QQ Plot After Removing Two Sinusoids. 


\subsection{Summary}

The analysis focused on estimating and removing one, two and four sinusoidal interference from the data segments using all estimated parameters and truncated peak method. Moreover, the fluctuation in frequency and magnitude in each segment has affected the performance of the tube average frequency and tube average frequency and magnitude methods. In terms of power and correlation, the removal of one, two and four sinusoidal interference using all estimated parameters for each segment has reduced the power of the original segment while keeping high correlation with the flaw signal. The QQ-plot in the residual analysis reveals that the leftover from the data after removing the sinusoidal interference tends toward normal distribution. As a result, using all estimated parameters for each segment with the two sinusoids estimation and rectangular windowing approach truncated peak method has showed better performance over other methods. 


\section{CHAPTER 6 CONCLUSION AND FUTURE}

\section{WORK}

\subsection{Conclusion}

A multistage approach of preprocessing and estimating the low-frequency sinusoidal interference parameters was employed with the aim of removing the low-frequency sinusoidal interference.

This research work focused on estimating and removing the low frequency sinusoidal noise. This process consists of three stages: signal preprocessing, estimation and removal of sinusoidal interference. The preprocessing steps extract the meaningful information and get the data ready for estimation. Many steps are involved in the signal preprocessing, background noise removal, artefacts removal, smoothing, and data segmentation. The estimation of the initial conditions involves the use of MUSIC algorithm, linear regression, least square estimates. The actual estimation involves the use Cyclic-MUSIC algorithm which is a modified version of the Relaxed algorithm and FFT.

The removal process involves generating sinusoidal waveforms from the estimated sinusoidal parameters and subtracted from the original data. Three methods have been examined, in terms of power and correlation; using all the estimated parameters technique has shown better 
performance over other methods. QQ-plot analysis revealed that the residuals from the data records tend toward normal distribution.

Estimation and removal of sinusoidal interference is a preparation step toward suppressing the support plates' response and filtering out the random noise.

\subsection{Future Work}

The general problem of eddy-current inspection data analysis is complex due to the variability of the noise and interference signals under different conditions. Designing and implementing a full automatic eddy current data analyzer is the prime goal. Many new techniques can be used and new improvement can be done to achieve the prime objective. Future works can be summarized as follows:

- Determining the number of sinusoids in each segment

- Signal processing techniques can be used to study the response of the support plates and suppress them.

- Random noise can be filtered out using adaptive filtering

- Classification process can be designed and implemented using DSP algorithms. 


\section{Appendix A}

\section{FORWARD BACKWARD PREDICTION}

The sample covariance matrix is used for eigenanalysis by many algorithms like MUSIC, Min-Norm and ESPRIT. The sample covariance $\hat{R}[23]$ :

$$
\hat{R}=\frac{1}{N} \sum_{t=m}^{N}\left[\begin{array}{c}
y(t) \\
\vdots \\
y(t-m+1)
\end{array}\right]\left[y *(t) \cdots y^{*}(t-m+1)\right]
$$

The $\hat{R}$ above is recognized to be the matrix that appears in the least squares (LS) estimation of the coefficients $\left\{\alpha_{K}\right\}$ of an $m^{\text {th }}$ order forward linear predictor of $\mathrm{y}^{*}(\mathrm{t}+1)$ :

$$
\hat{y}^{*}(t+1)=\alpha_{1} y^{*}(t)+\ldots+\alpha_{m} y^{*}(t-m+1)
$$

For this reason, the methods which obtain frequency estimates from $\hat{R}$ are named forward (F) approaches.

Frequency estimation accuracy can be enhanced by using the following modified sample covariance matrix, in lieu of $\hat{R}$,

$$
\hat{R}=\frac{1}{2}\left(\hat{R}+J \hat{R}^{T} J\right)
$$

where

$$
J=\left[\begin{array}{lll}
0 & . & 1 \\
1 & \cdot & 0
\end{array}\right]
$$


is the so-called exchange or reversal matrix. The second term in (A.3) has the following detailed form:

$$
J \hat{R}^{T} J=\frac{1}{N} \sum_{t=m}^{N}\left[\begin{array}{c}
y^{*}(t-m+1) \\
\vdots \\
y^{*}(t)
\end{array}\right][y(t-m+1) \cdots y(t)]
$$

The matrix (A.4) is the one that appears in the LS estimate of the coefficients of an $m^{\text {th }}$ order backward linear predictor of $\mathrm{y}(\mathrm{t}-\mathrm{m})$ :

$$
\hat{y}(t-m)=\mu_{1} y(t-m+1)+\ldots+\mu_{m} y(t)
$$

This observation, along with the previous remark made about R, suggests the name of forward-backward (FB) approaches for methods that determine frequency estimates from $\mathrm{R}$ in (A.3) [20].

The $(\mathrm{i}, \mathrm{j})$ element of $\widetilde{R}$ is given by:

$$
\widetilde{R}_{i, j}=\frac{1}{2 N} \sum_{t=m}^{N}\left[y(t-i) y *(t-j)+y^{*}(t-m+1+i) y(t-m+1+j)\right]
$$




\section{Appendix B}

\section{MUSIC and ROOT-MUSIC ALGORITHIMS}

From the covariance model matrix $\hat{R}[22]$ :

$$
\hat{R}=E\left\{\widetilde{y}(t) \tilde{y}^{*}(t)\right\}=A P A^{*}+\sigma I \quad ; \quad P=\left[\begin{array}{lll}
\sigma_{1}^{2} & & 0 \\
& \ddots & \\
0 & & \sigma_{n 1}^{2}
\end{array}\right]
$$

Let $\lambda_{1} \geq \lambda_{2} \geq \ldots \ldots \geq \lambda_{M}$ denote the eigenvalues of $\hat{R}$ and arranged in non-increasing order, and let $\left\{S_{1}, \ldots . . S_{n}\right\}$ be the orthonormal eigenvectors associated with $\left\{\lambda_{1}, \ldots \ldots, \lambda_{n}\right\}$ and $\left\{g_{1}, \ldots \ldots, g_{n-m}\right\}$ a set of orthonormal eigenvectors corresponding to $\left\{\lambda_{n+1}, \ldots \ldots, \lambda_{m}\right\}$

$$
\operatorname{rank}\left(A P A^{*}\right)=n
$$

It follows that

$$
\begin{aligned}
& \lambda_{k}>\sigma \quad \text { for } i=1, \ldots \ldots, n \text { and } \\
& \lambda_{k}=\sigma \quad \text { for } i=n+1, \ldots \ldots, m
\end{aligned}
$$

The set of eigenvalues of $\mathrm{R}$ can hence be split into two subsets.

Let $S=\left[s_{1}, \ldots \ldots, s_{n}\right] \quad(m \times n), \quad G=\left[g_{1}, \ldots \ldots, g_{m-n}\right] \quad(m \times(m-n))$

We observe that

$$
R G=A P A^{*} G+\sigma G=\sigma G
$$

Which implies that

$$
A^{*} G=0
$$

Which equivalent to:

$$
a^{*}(w) G G^{*} a(w)=0 \quad \text { for } \quad w=w_{1}, \ldots \ldots, w_{n}
$$


Where $a(w)=\left[1, e^{i w_{1}}, \cdots \cdots \cdot, e^{i w_{n}}\right]$

Since the normalized eigenvectors $\left\{s_{k}, g_{k}\right\}$ are orthonormal:

$$
S S^{*}+G G^{*}=I
$$

It follows that (B.4) can also be rewritten using (B.5) as:

$$
a^{*}(w)\left[I-S S^{*}\right] a(w)=0 \quad \text { for } w=w_{1} \ldots . ., w_{n} \quad \text { B.6 }
$$

We can see that the true parameter values $\left\{w_{1}, \ldots \ldots, w_{n}\right\}$ are the only solutions (B.4) and (B.6). The proof is by contradictions.

The music algorithm uses the following procedure to estimate the frequencies:

Step 1: compute the sample covariance matrix [23]

$$
\hat{R}=\frac{1}{N} \sum_{t=m}^{N} \tilde{y}(t) \widetilde{y}^{*}(t)
$$

and its eigen-decomposition. Let $\hat{S}$ and $\widehat{G}$ denote the matrices defined similarly to $\mathrm{S}$ and G, but made from eigenvectors $\left\{\hat{S}_{1}, \ldots \ldots, \hat{S}_{n}\right\}$ and $\left\{\hat{g}_{1}, \ldots \ldots, \hat{g}_{n}\right\}$ of $\hat{R}$.

Step 2: Determine frequency estimates as the locations of the $n$ highest peaks of the function

$$
\frac{1}{a^{*}(w) \hat{G} \hat{G}^{*} a(w)}, \quad w \in[-\pi, \pi] \quad \text { B. } 8
$$

This is called a pseudo-spectrum since it indicates the presence of sinusoidal components in the signal 


\section{ROOT-MUSIC}

Step 3: Determine frequency estimates as the angular positions of the $\mathrm{n}$ (pairs of reciprocal)

roots of the equation

$a^{T}\left(z^{-1}\right) \hat{G} \hat{G}^{*} a(z)=0$

B. 9

Which are located nearest the unit circle. And a(z) stands for the vector a(w) with $e^{i w}$ replaced by $\mathrm{z}$,

$a(z)=\left[1, z^{-1}, \ldots \ldots, z^{-(m-1)}\right]^{T}$

B. 10 


\section{Appendix C}

\section{ESTIMATION TABLES}

TUBE 3: One Sinusoid

\begin{tabular}{|c|c|c|c|}
\hline Segment \# & $\begin{array}{c}\text { Frequency } \\
\text { F1(MH) }\end{array}$ & $\begin{array}{c}\text { Magnitude } \\
\text { M1(V) }\end{array}$ & $\begin{array}{c}\text { Phase } \\
\text { Ph1(Radians) }\end{array}$ \\
\hline 1 & 6.18 & 0.23 & 0.71 \\
\hline 2 & 5.72 & 0.06 & -1.90 \\
\hline 3 & 8.72 & 0.05 & -0.42 \\
\hline 4 & 7.88 & 0.09 & -2.98 \\
\hline 5 & 18.88 & 0.08 & -0.82 \\
\hline 6 & 19.60 & 0.08 & -1.56 \\
\hline 7 & 7.76 & 0.06 & -0.62 \\
\hline 8 & 6.87 & 0.10 & 1.68 \\
\hline 9 & 7.77 & 0.24 & -1.36 \\
\hline 10 & 6.91 & 0.23 & -1.93 \\
\hline 11 & 5.91 & 0.15 & 0.95 \\
\hline 12 & 6.29 & 0.08 & 0.33 \\
\hline 13 & 5.15 & 0.13 & -2.65 \\
\hline 14 & 8.49 & 0.05 & -2.20 \\
\hline 15 & 5.98 & 0.28 & -0.78 \\
\hline 16 & 7.46 & 0.18 & -2.28 \\
\hline 17 & 6.15 & 0.21 & 2.76 \\
\hline 18 & 7.28 & 0.45 & 2.92 \\
\hline
\end{tabular}

TUBE 3: Two Sinusoids

\begin{tabular}{|c|c|c|c|c|c|c|}
\hline $\begin{array}{c}\text { Segment } \\
\#\end{array}$ & $\begin{array}{c}\text { Frequency } \\
\text { F1(Hz) }\end{array}$ & $\begin{array}{c}\text { Frequency } \\
\text { F2(Hz) }\end{array}$ & $\begin{array}{c}\text { Magnitude } \\
\text { M1(V) }\end{array}$ & $\begin{array}{c}\text { Magnitude } \\
\text { M2(V) }\end{array}$ & $\begin{array}{c}\text { Phase Ph1 } \\
\text { (Radians) }\end{array}$ & $\begin{array}{c}\text { Phase } \\
\text { Ph2 } \\
\text { (Radians) }\end{array}$ \\
\hline 1 & 7.04 & 6.07 & 0.19 & 0.25 & -0.27 & 0.95 \\
\hline 2 & 9.13 & 5.80 & 0.03 & 0.05 & 1.15 & -2.05 \\
\hline 3 & 19.94 & 8.74 & 0.05 & 0.05 & -2.42 & -0.42 \\
\hline 4 & 8.29 & 7.01 & 0.05 & 0.02 & 1.96 & -0.09 \\
\hline 5 & 18.88 & 5.64 & 0.08 & 0.06 & -0.80 & -2.90 \\
\hline 6 & 19.53 & 6.85 & 0.09 & 0.02 & -1.46 & 1.59 \\
\hline 7 & 8.36 & 6.45 & 0.05 & 0.03 & -2.21 & 0.20 \\
\hline 8 & 8.27 & 6.51 & 0.10 & 0.11 & -1.79 & -2.19 \\
\hline 9 & 9.35 & 7.87 & 0.06 & 0.22 & 1.13 & -1.71 \\
\hline 10 & 9.55 & 7.65 & 0.17 & 0.16 & 0.70 & -0.55 \\
\hline 11 & 7.12 & 4.97 & 0.15 & 0.16 & 0.68 & -0.50 \\
\hline 12 & 19.91 & 6.32 & 0.09 & 0.07 & -2.12 & 0.21 \\
\hline 13 & 19.83 & 5.15 & 0.08 & 0.13 & -2.87 & -2.66 \\
\hline 14 & 19.35 & 8.50 & 0.13 & 0.05 & -3.05 & -2.23 \\
\hline 15 & 10.09 & 5.96 & 0.13 & 0.28 & -0.83 & -0.72 \\
\hline 16 & 9.24 & 6.42 & 0.14 & 0.08 & 0.32 & 0.37 \\
\hline 17 & 9.73 & 5.57 & 0.18 & 0.15 & -1.03 & 3.01 \\
\hline 18 & 13.79 & 6.58 & 0.22 & 0.47 & 1.93 & -0.25 \\
\hline
\end{tabular}


TUBE 4: One Sinusoid

\begin{tabular}{|c|c|c|c|}
\hline $\begin{array}{c}\text { Segment } \\
\#\end{array}$ & $\begin{array}{c}\text { Frequency } \\
\text { F1(MH) }\end{array}$ & $\begin{array}{c}\text { Magnitude } \\
\text { M1(V) }\end{array}$ & $\begin{array}{c}\text { Phase } \\
\text { Ph1(Radians) }\end{array}$ \\
\hline 1 & 6.36 & 0.06 & 2.42 \\
\hline 2 & 6.07 & 0.11 & -1.75 \\
\hline 3 & 8.12 & 0.08 & 0.45 \\
\hline 4 & 9.21 & 0.11 & 0.36 \\
\hline 5 & 19.95 & 0.10 & -3.00 \\
\hline 6 & 6.70 & 0.04 & -0.41 \\
\hline 7 & 14.11 & 0.08 & 0.76 \\
\hline 8 & 26.72 & 0.10 & 1.31 \\
\hline 9 & 8.58 & 0.22 & 0.54 \\
\hline 10 & 6.16 & 0.18 & -1.53 \\
\hline 11 & 26.81 & 0.09 & -2.09 \\
\hline 12 & 7.07 & 0.05 & 2.47 \\
\hline 13 & 6.06 & 0.05 & -0.37 \\
\hline 14 & 6.99 & 0.07 & 1.70 \\
\hline 15 & 8.35 & 0.15 & 0.15 \\
\hline 16 & 5.56 & 0.12 & -2.92 \\
\hline 17 & 5.03 & 0.29 & 2.82 \\
\hline 18 & 6.43 & 0.13 & -1.21 \\
\hline
\end{tabular}

TUBE 4: Two Sinusoids

\begin{tabular}{|c|c|c|c|c|c|c|}
\hline $\begin{array}{c}\text { Segment } \\
\#\end{array}$ & $\begin{array}{c}\text { Frequency } \\
\text { F1(Hz) }\end{array}$ & $\begin{array}{c}\text { Frequency } \\
\text { F2(Hz) }\end{array}$ & $\begin{array}{c}\text { Magnitude } \\
\text { M1(V) }\end{array}$ & $\begin{array}{c}\text { Magnitude } \\
\text { M2(V) }\end{array}$ & $\begin{array}{c}\text { Phase Ph1 } \\
\text { (Radians) }\end{array}$ & $\begin{array}{c}\text { Phase Ph2 } \\
\text { (Radians) }\end{array}$ \\
\hline 1 & 14.14 & 6.04 & 0.06 & 0.07 & -0.79 & -2.50 \\
\hline 2 & 7.52 & 5.68 & 0.06 & 0.10 & -1.54 & 0.09 \\
\hline 3 & 19.57 & 7.86 & 0.08 & 0.07 & -3.05 & 1.13 \\
\hline 4 & 19.99 & 8.76 & 0.08 & 0.11 & -0.36 & 2.81 \\
\hline 5 & 19.96 & 7.93 & 0.10 & 0.07 & -3.01 & 1.45 \\
\hline 6 & 19.83 & 6.76 & 0.07 & 0.04 & 1.43 & -0.76 \\
\hline 7 & 14.20 & 7.35 & 0.08 & 0.06 & 0.49 & -0.29 \\
\hline 8 & 26.51 & 9.09 & 0.10 & 0.11 & 2.02 & 2.74 \\
\hline 9 & 7.24 & 5.77 & 0.18 & 0.19 & 2.00 & -1.87 \\
\hline 10 & 7.37 & 5.64 & 0.23 & 0.12 & -0.92 & -2.33 \\
\hline 11 & 26.70 & 14.50 & 0.09 & 0.07 & -1.79 & 1.17 \\
\hline 12 & 19.67 & 7.05 & 0.05 & 0.05 & -2.15 & 2.51 \\
\hline 13 & 12.61 & 5.81 & 0.04 & 0.03 & -0.67 & 0.54 \\
\hline 14 & 8.76 & 5.72 & 0.04 & 0.06 & -1.19 & 2.33 \\
\hline 15 & 8.64 & 5.24 & 0.11 & 0.19 & -1.42 & -0.78 \\
\hline 16 & 12.83 & 5.51 & 0.05 & 0.11 & 2.20 & -2.76 \\
\hline 17 & 8.38 & 4.80 & 0.16 & 0.32 & -0.98 & -2.85 \\
\hline 18 & 11.68 & 6.54 & 0.05 & 0.14 & 2.39 & -1.49 \\
\hline
\end{tabular}


TUBE 5: One Sinusoid

\begin{tabular}{|c|c|c|c|}
\hline Segment \# & $\begin{array}{c}\text { Frequency } \\
\text { F1(MH) }\end{array}$ & $\begin{array}{c}\text { Magnitude } \\
\text { M1(V) }\end{array}$ & $\begin{array}{c}\text { Phase } \\
\text { Ph1 } \\
\text { (Radians) }\end{array}$ \\
\hline 1 & 6.33 & 0.14 & -0.30 \\
\hline 2 & 5.25 & 0.03 & 0.41 \\
\hline 3 & 19.10 & 0.07 & 1.37 \\
\hline 4 & 18.88 & 0.09 & -0.28 \\
\hline 5 & 5.05 & 0.22 & 2.28 \\
\hline 6 & 19.09 & 0.09 & 0.19 \\
\hline 7 & 7.69 & 0.04 & 2.58 \\
\hline 8 & 5.74 & 0.13 & 2.77 \\
\hline 9 & 25.47 & 0.18 & 0.66 \\
\hline 10 & 5.74 & 0.06 & 2.15 \\
\hline 11 & 25.31 & 0.06 & -2.16 \\
\hline 12 & 19.14 & 0.06 & 2.33 \\
\hline 13 & 19.37 & 0.07 & -2.61 \\
\hline 14 & 6.85 & 0.11 & -2.79 \\
\hline 15 & 18.66 & 0.08 & -2.47 \\
\hline 16 & 6.00 & 0.06 & 0.50 \\
\hline 17 & 8.87 & 0.08 & 2.08 \\
\hline 18 & 10.52 & 0.12 & -2.32 \\
\hline
\end{tabular}

TUBE 5: Two Sinusoids

\begin{tabular}{|c|c|c|c|c|c|c|}
\hline Segment \# & $\begin{array}{c}\text { Frequency } \\
\text { F1(Hz) }\end{array}$ & $\begin{array}{c}\text { Frequency } \\
\text { F2(Hz) }\end{array}$ & $\begin{array}{c}\text { Magnitude } \\
\text { M1(V) }\end{array}$ & $\begin{array}{c}\text { Magnitude } \\
\text { M2(V) }\end{array}$ & $\begin{array}{c}\text { Phase Ph1 } \\
\text { (Radians) }\end{array}$ & $\begin{array}{c}\text { Phase Ph2 } \\
\text { (Radians) }\end{array}$ \\
\hline 1 & 9.51 & 5.99 & 0.07 & 0.18 & 2.63 & 0.48 \\
\hline 2 & 18.91 & 5.65 & 0.08 & 0.06 & -0.46 & -1.35 \\
\hline 3 & 19.11 & 9.77 & 0.07 & 0.03 & 1.32 & -0.03 \\
\hline 4 & 18.92 & 7.98 & 0.09 & 0.04 & -0.41 & 2.20 \\
\hline 5 & 9.10 & 4.99 & 0.14 & 0.23 & -0.28 & 2.47 \\
\hline 6 & 19.09 & 7.54 & 0.09 & 0.06 & 0.17 & 2.92 \\
\hline 7 & 8.66 & 6.48 & 0.05 & 0.04 & -0.20 & -0.71 \\
\hline 8 & 5.61 & 4.45 & 0.10 & 0.21 & -2.40 & -2.27 \\
\hline 9 & 25.49 & 8.10 & 0.18 & 0.13 & 0.58 & 2.49 \\
\hline 10 & 7.28 & 5.58 & 0.16 & 0.11 & -1.92 & 2.84 \\
\hline 11 & 25.30 & 12.53 & 0.06 & 0.04 & -2.09 & -0.81 \\
\hline 12 & 19.14 & 8.29 & 0.06 & 0.03 & 2.32 & -0.18 \\
\hline 13 & 19.38 & 5.83 & 0.07 & 0.02 & -2.65 & 1.14 \\
\hline 14 & 19.23 & 6.84 & 0.06 & 0.11 & -0.54 & -2.75 \\
\hline 15 & 18.66 & 8.65 & 0.08 & 0.02 & -2.46 & 1.45 \\
\hline 16 & 12.48 & 5.83 & 0.05 & 0.07 & 0.89 & 1.42 \\
\hline 17 & 18.76 & 8.82 & 0.07 & 0.08 & -1.55 & 2.15 \\
\hline 18 & 11.06 & 8.19 & 0.05 & 0.10 & -1.33 & -1.11 \\
\hline
\end{tabular}


TUBE 6: One Sinusoid

\begin{tabular}{|c|c|c|c|}
\hline Segment \# & $\begin{array}{c}\text { Frequency } \\
\text { F1(MH) }\end{array}$ & $\begin{array}{c}\text { Magnitude } \\
\text { M1(V) }\end{array}$ & $\begin{array}{c}\text { Phase Ph1 } \\
\text { (Radians) }\end{array}$ \\
\hline 1 & 6.25 & 0.12 & -3.09 \\
\hline 2 & 4.72 & 0.14 & -3.00 \\
\hline 3 & 19.49 & 0.12 & -0.75 \\
\hline 4 & 7.74 & 0.09 & -0.07 \\
\hline 5 & 6.00 & 0.12 & -0.08 \\
\hline 6 & 8.59 & 0.11 & -2.80 \\
\hline 7 & 19.18 & 0.09 & 1.03 \\
\hline 8 & 19.62 & 0.09 & -0.59 \\
\hline 9 & 5.98 & 0.13 & 2.86 \\
\hline 10 & 6.55 & 0.20 & 0.97 \\
\hline 11 & 6.02 & 0.18 & -2.07 \\
\hline 12 & 19.37 & 0.08 & 1.80 \\
\hline 13 & 19.20 & 0.11 & 1.62 \\
\hline 14 & 6.18 & 0.10 & -0.28 \\
\hline 15 & 6.71 & 0.04 & -0.33 \\
\hline 16 & 19.16 & 0.08 & -2.03 \\
\hline 17 & 6.60 & 0.13 & -2.63 \\
\hline 18 & 6.27 & 0.19 & -2.59 \\
\hline
\end{tabular}

TUBE 6: Two Sinusoids

\begin{tabular}{|c|c|c|c|c|c|c|}
\hline Segment \# & $\begin{array}{c}\text { Frequency } \\
\text { F1(Hz) }\end{array}$ & $\begin{array}{c}\text { Frequency } \\
\text { F2(Hz) }\end{array}$ & $\begin{array}{c}\text { Magnitude } \\
\text { M1(V) }\end{array}$ & $\begin{array}{c}\text { Magnitude } \\
\text { M2(V) }\end{array}$ & $\begin{array}{c}\text { Phase Ph1 } \\
\text { (Radians) }\end{array}$ & $\begin{array}{c}\text { Phase Ph2 } \\
\text { (Radians) }\end{array}$ \\
\hline 1 & 7.74 & 5.50 & 0.06 & 0.12 & 0.57 & 0.10 \\
\hline 2 & 8.36 & 4.71 & 0.08 & 0.14 & 0.07 & -2.92 \\
\hline 3 & 19.49 & 8.00 & 0.12 & 0.04 & -0.77 & -1.97 \\
\hline 4 & 19.34 & 7.72 & 0.13 & 0.09 & -2.48 & 0.01 \\
\hline 5 & 14.97 & 6.01 & 0.09 & 0.12 & -2.37 & -0.12 \\
\hline 6 & 9.75 & 6.48 & 0.09 & 0.09 & 1.37 & 2.30 \\
\hline 7 & 19.18 & 6.80 & 0.09 & 0.02 & 1.04 & -0.18 \\
\hline 8 & 19.64 & 5.75 & 0.08 & 0.10 & -0.66 & 0.45 \\
\hline 9 & 10.08 & 6.42 & 0.07 & 0.13 & -1.52 & 0.55 \\
\hline 10 & 10.01 & 6.72 & 0.08 & 0.16 & 0.01 & 0.13 \\
\hline 11 & 19.26 & 6.07 & 0.07 & 0.16 & -1.22 & -2.24 \\
\hline 12 & 19.28 & 6.62 & 0.08 & 0.06 & 2.06 & -0.55 \\
\hline 13 & 19.30 & 8.57 & 0.11 & 0.10 & 1.30 & -1.44 \\
\hline 14 & 7.26 & 5.06 & 0.15 & 0.11 & 1.14 & -1.54 \\
\hline 15 & 19.27 & 6.78 & 0.09 & 0.04 & -1.47 & -0.34 \\
\hline 16 & 19.17 & 8.40 & 0.08 & 0.03 & -2.06 & -1.93 \\
\hline 17 & 9.19 & 6.47 & 0.08 & 0.14 & -2.30 & -2.33 \\
\hline 18 & 11.54 & 6.28 & 0.12 & 0.19 & -0.67 & -2.62 \\
\hline
\end{tabular}


TUBE 7: One Sinusoid

\begin{tabular}{|c|c|c|c|}
\hline Segment \# & $\begin{array}{c}\text { Frequency } \\
\text { F1(MH) }\end{array}$ & $\begin{array}{c}\text { Magnitude } \\
\text { M1(V) }\end{array}$ & $\begin{array}{c}\text { Phase Ph1 } \\
\text { (Radians) }\end{array}$ \\
\hline 1 & 6.09 & 0.08 & -2.67 \\
\hline 2 & 5.23 & 0.06 & -1.66 \\
\hline 3 & 7.31 & 0.12 & -1.32 \\
\hline 4 & 10.30 & 0.16 & 2.23 \\
\hline 5 & 14.75 & 0.13 & -0.28 \\
\hline 6 & 7.30 & 0.17 & 1.23 \\
\hline 7 & 8.41 & 0.01 & -2.45 \\
\hline 8 & 5.84 & 0.06 & 2.45 \\
\hline 9 & 10.47 & 0.05 & 2.46 \\
\hline 10 & 6.56 & 0.35 & -1.41 \\
\hline 11 & 6.41 & 0.25 & 1.01 \\
\hline 12 & 6.62 & 0.17 & -0.47 \\
\hline 13 & 6.64 & 0.26 & 0.03 \\
\hline 14 & 6.20 & 0.12 & -2.81 \\
\hline 15 & 5.35 & 0.11 & -2.93 \\
\hline 16 & 6.68 & 0.13 & -0.82 \\
\hline 17 & 7.32 & 0.15 & 2.90 \\
\hline 18 & 5.64 & 0.12 & 0.53 \\
\hline
\end{tabular}

TUBE 7: Two Sinusoids

\begin{tabular}{|c|c|c|c|c|c|c|}
\hline Segment \# & $\begin{array}{c}\text { Frequency } \\
\text { F1(Hz) }\end{array}$ & $\begin{array}{c}\text { Frequency } \\
\text { F2(Hz) }\end{array}$ & $\begin{array}{c}\text { Magnitude } \\
\text { M1(V) }\end{array}$ & $\begin{array}{c}\text { Magnitude } \\
\text { M2(V) }\end{array}$ & $\begin{array}{c}\text { Phase Ph1 } \\
\text { (Radians) }\end{array}$ & $\begin{array}{c}\text { Phase Ph2 } \\
\text { (Radians) }\end{array}$ \\
\hline 1 & 15.31 & 6.12 & 0.09 & 0.08 & -0.52 & -2.79 \\
\hline 2 & 12.60 & 5.07 & 0.04 & 0.07 & 1.11 & -0.76 \\
\hline 3 & 14.84 & 7.39 & 0.08 & 0.12 & 2.51 & -1.50 \\
\hline 4 & 11.05 & 7.84 & 0.14 & 0.10 & 0.97 & -1.01 \\
\hline 5 & 14.77 & 7.85 & 0.13 & 0.04 & -0.38 & -2.70 \\
\hline 6 & 14.79 & 7.29 & 0.10 & 0.17 & -0.06 & 1.25 \\
\hline 7 & 14.08 & 8.76 & 0.05 & 0.04 & 0.17 & -0.48 \\
\hline 8 & 24.81 & 5.68 & 0.11 & 0.05 & 1.09 & 2.93 \\
\hline 9 & 12.27 & 10.67 & 0.08 & 0.05 & 1.26 & 1.41 \\
\hline 10 & 6.54 & 5.92 & 0.53 & 0.40 & -1.48 & -2.97 \\
\hline 11 & 8.26 & 5.20 & 0.13 & 0.23 & -2.09 & 0.98 \\
\hline 12 & 8.94 & 6.00 & 0.07 & 0.13 & 1.46 & -0.44 \\
\hline 13 & 9.34 & 6.44 & 0.10 & 0.22 & 2.49 & 0.61 \\
\hline 14 & 6.49 & 4.21 & 0.17 & 0.07 & 2.98 & 1.10 \\
\hline 15 & 10.09 & 5.36 & 0.01 & 0.11 & -2.06 & -2.95 \\
\hline 16 & 8.62 & 6.06 & 0.04 & 0.06 & 1.27 & 1.26 \\
\hline 17 & 7.92 & 6.03 & 0.14 & 0.15 & 0.41 & -0.71 \\
\hline 18 & 11.21 & 5.65 & 0.18 & 0.11 & -1.45 & 0.55 \\
\hline
\end{tabular}




\section{References}

[1] W. J. McGonnagle, Nondestructive Testing, Gordon and Breach Science Publishers, New York, 1982.

[2] R. Lakhan. Non-destructive testing and eddy current data. Presented at Carleton University Campus. Ottawa, 2013.

[3] CANDU Nuclear Reactors, [Image] Nuclear Reactor. Retrieved August 19, 2013, from Canadian Nuclear Association website http://www.cna.ca/how_works/candu_technology/

[4] CANDU Steam Generator [Image] William Snook, 2001.07.20, CD to WJG, image 970678-2 Stm Gen.pdf, Retrieved August 20, 2013, from https://canteach.candu.org/Image\%20Library1/Forms/DispForm.aspx?ID=30\&RootFolde $\mathrm{r}=\% 2$ fImage $\% 20$ Library $1 \% 2 \mathrm{f} 33000$

[5] S. S. Udpa, L. Udpa, "Eddy Current Nondestructive Evaluation", Wiley Encyclopedia of Electrical and Electronics Engineering, edited by John G. Webster, Vol. 6, pp. 149-163, 1999

[6] S. Udpa "Automated Analysis of Bobbin Coil Probe Eddy Current Data", EPRI, Palo Alto, CA: 2002.

[7] R. B. Thompson and D. O. Thompson, "Ultrasonics in Nondestructive Evaluation", Proceedings of the IEEE, Vol. 73, No. 12, Dec 1985.

[8] Louis Cartz, "Nondestructive Testing: Radiography, Ultrasonics, Liquid Penetrant, Magnetic Particle, Eddy Current", ASM Intl; ISBN: 0871705176.

[9] W. Lord and R. Palanisamy, "Development of theoretical Models for NDT eddy current Phenomena", Symposium on Eddy Current Characterization of Materials and Structures, NBS, Gaithersburg, Maryland, September 1979.

[10] R. C. McMaster, P. McIntire and M. L. Mester, Nondestructive testing handbook, Vol. 4, 2nd Ed., American Society for Nondestructive Testing, Columbus, Ohio, 1968.

[11] H. L. Libby, Introduction to electromagnetic nondestructive test methods, WileyInterscience, New York, 1971.

[12] Y. Sun, T. Ouang, S. Udpa, and Z. Zeng, "Detecting Compressive Residual Stress in Carbon Steel Specimens of Flat Geometries Using the Remote-Field Eddy Current Technique", Review of Progress in Quantitative Nondestructive Evaluation, Maine, USA, July 2001. 
[13] W. Lord, Electromagnetic methods of nondestructive testing, Gordon and Breach Science Publishers, London, 1985, pp. 175-304.

[14] R. C. McMaster, P. McIntire and M. L. Mester, Nondestructive testing handbook, Vol. 4, 2nd Ed., American Society for Nondestructive Testing, Columbus, Ohio, 1968.

[15] S. Bakhitiari and D. S. Kupperman, "Assessment of Noise Level of Eddy Current Inspection of Steam Generator Tubes", NUREG/CR-6982 ANL-05/44(March 2009).

[16] H. Shekhar, R. Polikar, P. Ramuhalli, X. Liu, M. Das, L. Udpa and S.S. Udpa, "Dynamic thresholding for automated analysis of bobbin probe eddy current data", International Journal of Applied Electromagnetics and Mechanics 15 (2001/2002) 39-46.

[17] T.K. Moon and W.C. Stirling, Mathematical Methods and Algorithms for Signal Processing, Prentice Hall, Upper Saddle River, NJ, 2000.

[18] M. Da1, P. Ramuhalli, L Udpa, S. Udpa, "An adaptive wiener filter based technique for automated detection of defect locations from bobbin coil eddy current data", Review of Quantitative Nondestructive Evaluation Vol. 21, 2002 American Institute of Physics 07354-006 1-5C.

[19] Li, J., and Stoica, P, Abramovich, Yu.I.: "On using cyclic algorithms for sinusoidal parameters estimation", Electronics letters., 2008, 44, (19).

[20] Li, J., and Stoica, P.: "Efficient mixed-spectrum estimation with applications to target feature extraction”, IEEE Trans. Signal Process., 1996, 44, (2), pp. 281-295.

[21] Abramovich, Yu.I.: "A recursion method for resolution of wideband signals having substantially different intensities", Radiotekhn. Elektron. (Translation: Radio Engineering and Electronic Physics), 1979, 23, (8), pp. 66-70.

[22] Shaw, A.K.: "A novel cyclic algorithm for maximum likelihood frequency estimation.", IEEE Int. Conf. on Systems Engineering, Dayton, OH, USA, August 1991, pp. 412-415

[23] J. Hwang and Y. Chen, "Superresolution frequency estimation by alternating notch periodogram," IEEE Trans. Signal Processing, vol. 41, no. 2, pp. 727-741, Feb. 1993.

[24] Marple, S.L. Digital Spectral Analysis, Englewood Cliffs, NJ, Prentice-Hall, 1987, pp. $373-378$.

[25] Stoica, P., and R.L. Moses, Introduction to Spectral Analysis, Prentice-Hall, Englewood Cliffs, NJ, 1997.

[24] P. Stoica and A. Nehorai, "MUSIC, maximum likelihood, and Cramer Rao bound", IEEE Trans. Acoust. Speech, Signal Processing,vol. ASSP-37, pp. 720-741, May 1989. 
[27] S. F. Yau and Y. Bresler, "A compact Cram\&-Rao bound expression for parametric estimation of superimposed signals", IEEE Trans. Signal Processing, vol. 40, no. 5, pp. 1226-1230, May 1992.

[28] Stoica, P. and A. Nehora. "MUSIC, Maximum Likelihood, and Cramer-Rao Bound: Further Results and Comparisons.", Technical Report 8819, Center for Systems Science, Yale University, New Haven, CT, Sept. 1988.

[29] Stoica, P. and A. Nehorai. "MODE, Maximum Likelihood, and Cramer-Rao Bound: Conditional and Unconditional Results.", Technical Report 8901, Center for Systems Science, Yale University, New Haven, CT, Jan. 1989.

[30] S. Kay, Fundamentals of Statistical Signal Processing: Estimation Theory. Prentice-Hall International Editions, Englewood Cliffs, NJ, 1993. 